\title{
Automatically Verified Mechanized Proof of One-Encryption Key Exchange
}

\author{
Bruno Blanchet \\ INRIA, École Normale Supérieure, CNRS \\ Paris, France \\ bruno.blanchet@inria.fr
}

\begin{abstract}
We present a mechanized proof of the passwordbased protocol One-Encryption Key Exchange (OEKE) using the computationally-sound protocol prover CryptoVerif. OEKE is a non-trivial protocol, and thus mechanizing its proof provides additional confidence that it is correct. This case study was also an opportunity to implement several important extensions of CryptoVerif, useful for proving many other protocols. We have indeed extended CryptoVerif to support the computational Diffie-Hellman assumption. We have also added support for proofs that rely on Shoup's lemma and additional game transformations. In particular, it is now possible to insert case distinctions manually and to merge cases that no longer need to be distinguished. Eventually, some improvements have been added on the computation of the probability bounds for attacks, providing better reductions. In particular, we improve over the standard computation of probabilities when Shoup's lemma is used, which allows us to improve the bound given in a previous manual proof of OEKE, and to show that the adversary can test at most one password per session of the protocol. In this paper, we present these extensions, with their application to the proof of OEKE. All steps of the proof are verified by CryptoVerif.

This document is an updated version of a report from 2012. In the 10 years between 2012 and 2022, CryptoVerif has made a lot of progress. In particular, the probability bound obtained by CryptoVerif for OEKE has been improved, reaching an almost optimal probability: only statistical terms corresponding to collisions between group elements or between hashes are overestimated by a small constant factor.
\end{abstract}

Keywords-Automatic proofs, Formal methods, Provable security, Protocols, Password-based authentication

\section{INTRODUCTION}

Since the beginning of public-key cryptography, more and more complex security notions have been defined, with protocols getting also more intricate. Initially, a long time without attack was a good argument in favor of the security of a scheme. But some schemes took a long time before being broken. A famous example is the Chor-Rivest cryptosystem [1], [2], which took more than 10 years to be totally broken [3]. Nowadays, the lack of attacks is no longer considered as a security validation, and provable security is a requirement for any new proposal.

The basic idea of provable security consists in reducing a well-known hard problem to an attack, in the complexity theory framework. Such a reduction guarantees that an efficient adversary against the cryptosystem could be converted into an efficient algorithm against the hard problem. First security proofs were essentially theoretical, providing polynomial reductions only. But "exact security" [4] or "concrete security" [5] asked for more efficient reductions.

Unfortunately, a security result should be considered with care. As explained above, it consists of a theorem which states that under a precise intractability assumption a specific security model (goals and means of the adversary) is satisfied. The reduction constitutes the proof of the theorem. Weaknesses can appear at several steps: the intractability assumption can be too strong, or even wrong; the security model might not correspond to the expected security level; the reduction may not be tight; and the proof can be erroneous. Because of more and more complex security models and proofs, most of them are never (double)-checked.

A famous example is the OAEP construction [6] that has been proven to achieve chosen-ciphertext security. But because of ambiguous security models in the early 90s, the difference between the so-called IND-CCA1 and IND-CCA2 security levels was not explicit. As a consequence, the proof was believed to achieve the IND-CCA2 level, until Shoup [7] exhibited a counter-example. Fortunately, a complete proof for IND-CCA2 has quickly been provided [8]. A machinechecked proof has later been provided [9].

As suggested by Halevi [10], computers could help in verifying proofs. This paper follows this path, with computationally-sound computer-aided proof and verification of cryptographic protocols.

Related Work: Various methods have been proposed for reaching Halevi's goal. Following the seminal paper by Abadi and Rogaway [11], many results show the soundness of the Dolev-Yao model with respect to the computational model, which makes it possible to use Dolev-Yao provers in order to prove protocols in the computational model (see, e.g., [12], [13], [14], [15], [16] and the survey [17]). However, these results have limitations, in particular in terms of allowed cryptographic primitives (they must satisfy strong security properties so that they correspond to DolevYao style primitives), and they require some restrictions on protocols (such as the absence of key cycles). A tool [18] was developed based on [12] to obtain computational proofs 
using the formal verifier AVISPA, for protocols that rely on public-key encryption and signatures.

Several frameworks exist for formalizing proofs of protocols in the computational model. Backes, Pfitzmann, and Waidner [19], [20] designed an abstract cryptographic library and showed its soundness with respect to computational primitives, under arbitrary active attacks. This framework has been used for a computationally-sound machine-checked proof of the Needham-Schroeder-Lowe protocol [21], [22]. Canetti [23] introduced the notion of universal composability. With Herzog [24], they show how a Dolev-Yao-style symbolic analysis can be used to prove security properties of protocols within the framework of universal composability, for a restricted class of protocols using publickey encryption as only cryptographic primitive. Then, they use the automatic Dolev-Yao verification tool ProVerif [25] for verifying protocols in this framework. Process calculi have been designed for representing cryptographic games, such as the probabilistic polynomial-time calculus of [26] and the cryptographic lambda-calculus of [27]. Logics have also been designed for proving security protocols in the computational model, such as the computational variant of PCL (Protocol Composition Logic) [28], [29] and CIL (Computational Indistinguishability Logic) [30]. Canetti et al. [31] use the framework of time-bounded task-PIOAs (Probabilistic Input/Output Automata) to prove security protocols in the computational model. This framework makes it possible to combine probabilistic and non-deterministic behaviors. These frameworks can be used to prove security properties of protocols in the computational sense, but except for [24] which relies on a Dolev-Yao prover, they have not been automated up to now, as far as we know.

Several techniques have been used for directly mechanizing proofs in the computational model. Type systems [32], [33], [34], [35] provide computational security guarantees. For instance, [32] handles shared-key and public-key encryption, with an unbounded number of sessions, by relying on the Backes-Pfitzmann-Waidner library. A type inference algorithm is given in [36]. In another line of research, a specialized Hoare logic was designed for proving asymmetric encryption schemes in the random oracle model [37], [38].

The tool CertiCrypt [39], [40], [41], [42], [9] enables the machine-checked construction and verification of cryptographic proofs by sequences of games [43], [44]. It relies on the general-purpose proof assistant Coq, which is widely believed to be correct. Nowak et al. [45], [46], [47] follow a similar idea by providing Coq proofs for several cryptographic primitives. EasyCrypt [48], the successor of CertiCrypt, provides more automation by relying on SMT solvers, which makes the tool easier to use, but still requires the user to provide the sequence of games and hints of why they are indistinguishable.

Independently, we have built the tool CryptoVerif [49] to help cryptographers, not only for the verification, but also by generating the proofs by sequences of games [43], [44], automatically or with little user interaction. The games are formalized in a probabilistic polynomial-time process calculus. CryptoVerif provides a generic method for specifying security properties of many cryptographic primitives. It proves secrecy and authentication properties. It also provides a bound on the probability of success of an attack. It has already been used to prove several cryptographic protocols, and also primitives [50]. This tool extends considerably early work by Laud [51], [52] which was limited either to passive adversaries or to a single session of the protocol. More recently, Tšahhirov and Laud [53], [54] developed a tool similar to CryptoVerif but that represents games by dependency graphs. It handles public-key and shared-key encryption and proves secrecy properties; it does not provide bounds on the probability of success of an attack.

Contributions: In this paper, we use the tool CryptoVerif in order to prove the password-based key exchange protocol One-Encryption Key-Exchange (OEKE) [55], a variant of Encrypted Key Exchange (EKE) [56]. This is a non-trivial case study, since EKE was not proved correct before 2003, 10 years after its publication. This mechanized proof provides additional confidence that the protocol OEKE is secure. More precisely, we have shown that OEKE guarantees the secrecy of the session key and the authentication of the client to the server. The proof is manually guided as detailed in Section IV. With the manual proof indications included in the CryptoVerif input file, the runtime of CryptoVerif version 2.06 for this proof was $2 \mathrm{~s}$ on an Intel Xeon $3.60 \mathrm{GHz}{ }^{1}$

This case study was also an opportunity for implementing several extensions of CryptoVerif, useful for proving many other protocols. Here are these extensions:

- CryptoVerif's specification mechanism for assumptions on primitives did not support the computational DiffieHellman (CDH) assumption, needed for proving OEKE and many important protocols. We have extended it to support CDH (Section III-D). This extension also allowed us to prove a signed Diffie-Hellman protocol, in a fully automatic way.

- We have extended CryptoVerif to be able to apply Shoup's lemma [43], by introducing events and later bounding their probability. We improve over the standard computation of probabilities, for applications of Shoup's lemma, by avoiding to count several times probabilities that in fact correspond to the same runs. This allows us to obtain better probability bounds than [55] and to show that the adversary can test at most one password per session of the client or the server, which is the optimal result. This improvement applies both to CryptoVerif proofs and to manual proofs, and it is not specific to the OEKE protocol (Section IV-C).

- Additional game transformations were also needed for

${ }^{1} \mathrm{~A}$ previous proof was done using CryptoVerif 1.14 [57], [58]. 
manually introducing case distinctions or for merging cases. We have implemented these transformations (Sections IV-C and IV-F).

- Password-based protocols require a careful computation of the probability of an attack, since one aims to compute how many passwords the adversary can test by interacting with the protocol. We have improved CryptoVerif in this respect (Section IV-G).

Outline: We recall the protocol OEKE in the next section. Section III presents the CryptoVerif model of the protocol, and Section IV presents its proof. We conclude in Section V. The appendices give background on CryptoVerif and additional details. The tool CryptoVerif and the input and output files can be found at https://cryptoverif.inria.fr/OEKE/.

Notations: $|S|$ denotes the cardinal of the set $S$. \#O denotes the number of calls to oracle $O$.

\section{THE OEKE PROTOCOL}

Password-authenticated key exchange protocols allow two parties that share a low-entropy common secret (a password) to agree on a common high-entropy secret key thereafter used with symmetric primitives, such as symmetric encryption for privacy and message authentication codes for authentication. The goal of such a protocol is to guarantee the secrecy of the resulting common key between the two participating players. Furthermore, the protocol should succeed if and only if the two players actually share the same password, which guarantees the identity of the partner to both of them. Because of the low-entropy, an active adversary will succeed in impersonating a party to the other one with nonnegligible probability by successive password guesses. Such an on-line dictionary attack is unavoidable. However, one should guarantee that this is the best attack: one active attack allows the adversary to test and thus eliminate at most one password, and not more. Namely, passive attacks should not (computationally) leak any information about the password. One definitely wants to prevent off-line dictionary attacks, where after a few active attacks and possibly many passive ones the collected information is enough to eliminate many passwords, and thus accelerate impersonation from the online dictionary attack.

The first password-authenticated key exchange protocol has been proposed by Bellovin and Merritt [56], the Encrypted Key Exchange (EKE). This is basically a DiffieHellman key exchange where the two flows are encrypted with a symmetric encryption scheme, using the password as secret key. Several variants have thereafter been proposed, such as AuthA [59]. The One-Encryption Key Exchange protocol (OEKE) studied in [55] is the particular variant where the second flow only is encrypted under the password, and the first player proves his knowledge of the password with an additional key confirmation flow. Figure 1 provides a description of this OEKE protocol, which guarantees client authentication and key secrecy, under the assumptions that

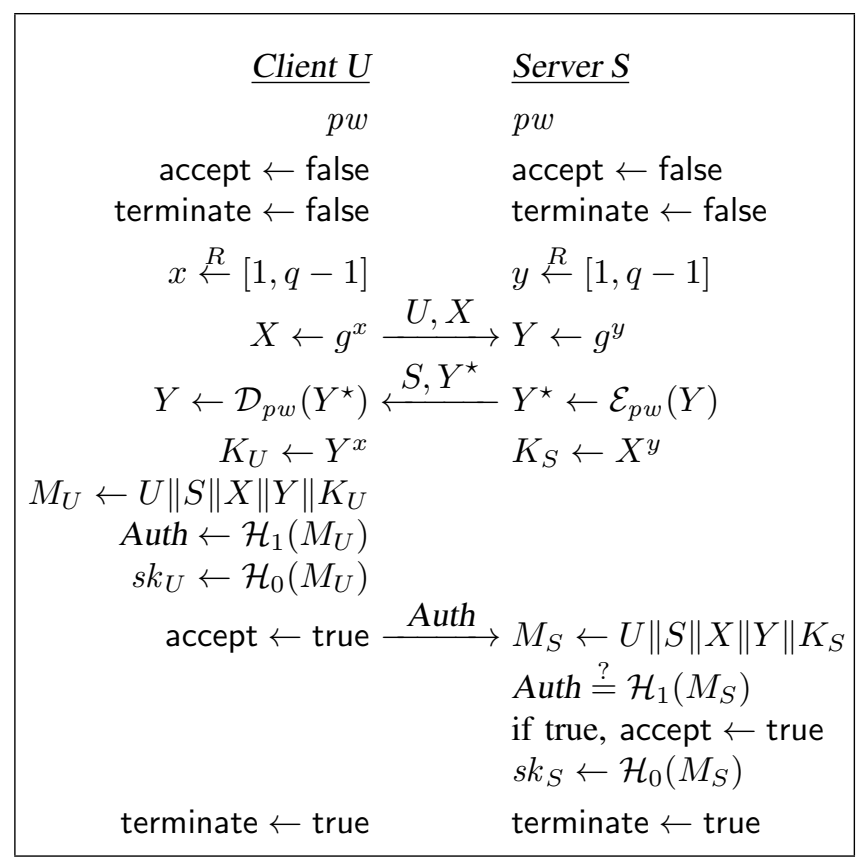

Figure 1. An execution of OEKE, run by client $U$ and server $S$. The session key is $s k=\mathcal{H}_{0}\left(U\|S\| X\|Y\| Y^{x}\right)=\mathcal{H}_{0}\left(U\|S\| X\|Y\| X^{y}\right)$.

$\mathcal{H}_{0}$ and $\mathcal{H}_{1}$ are random oracles, that $\mathcal{E}$ and $\mathcal{D}$ are respectively the encryption and decryption of an ideal cipher, and that $\mathbb{G}$ is a finite group of prime order $q$, with generator $g$, in which the computational Diffie-Hellman problem is hard (see the definition in Section III-D), as proven in [55]. If the password $p w$ is chosen among a finite dictionary passwd of size $N$ equipped with the uniform distribution, their proof shows that the probability for any adversary, within time $t$, and with less than $N_{U}$ sessions with a client, $N_{S}$ sessions with a server (active attacks) and $N_{P}$ sessions with passive eavesdropping (passive attacks), and, asking $q_{h}$ hash-queries and $q_{e}$ encryption/decryption queries, to make a server instance accept with no terminating client partner is bounded by

$$
\begin{aligned}
& \frac{N_{U}+2 N_{S}}{N}+3 q_{h} \times \operatorname{Succ}_{\mathbb{G}}^{\text {cdh }}\left(t^{\prime}\right)+p_{\text {coll }} \\
& \text { with } p_{\text {coll }}=\frac{\left(2 q_{e}+2 N_{U}+3 N_{S}+3 N_{P}\right)^{2}}{2(q-1)}+\frac{q_{h}^{2}+4 N_{S}}{2^{\ell_{1}+1}}
\end{aligned}
$$

where $\ell_{1}$ is the length of the output of $\mathcal{H}_{1}$ and $t^{\prime} \leq$ $t+\left(2 N_{U}+N_{S}+2 N_{P}+q_{e}+1\right) \cdot \tau_{\exp }$, with $\tau_{\exp }$ denoting the computation time for an exponentiation in $\mathbb{G}^{2}$ Furthermore, $\operatorname{Succ}_{\mathbb{G}}^{\text {cdh }}(t)$ denotes the maximal success probability an adversary can gain within time $t$ against the computational Diffie-Hellman problem in $\mathbb{G}$. Similarly, no adversary can

\footnotetext{
${ }^{2}$ In [55], they use as parameters the number $q_{s}$ of interactions with the parties instead of the numbers of sessions $N_{U}$ and $N_{S}$, and the number $q_{p}$ of passive eavesdroppings instead of the number of sessions $N_{P}$. It is straightforward to recompute the probabilities to use $N_{S}, N_{U}$, and $N_{P}$ instead, and this yields a more precise evaluation.
} 
distinguish the session key from a random key with advantage greater than

$$
\frac{2 N_{U}+4 N_{S}}{N}+8 q_{h} \times \operatorname{Succ}_{\mathbb{G}}^{\mathrm{cdh}}\left(t^{\prime}\right)+2 p_{\text {coll }} .
$$

The proofs basically show that the unique way for the adversary to gain something (against both client authentication and secrecy of the session key) is to correctly guess the password, by either sending a $Y^{\star}$ that is really an encryption under the correct password, or using the correct password to decrypt $Y^{\star}$ and compute the authenticator Auth. One could hope to prove that the former event, denoted Encrypt, is bounded by $N U / N$ and the latter event, denoted Auth, is bounded by $N S / N$. But, because of the way probabilities are computed when one uses Shoup's lemma [43], some factor appears to the $(N U+N S) / N$ main term.

\section{MOdELING OEKE IN CRYPTOVERIF}

In this section, we present the model of the protocol given as input to CryptoVerif. We first recall some basic ideas behind CryptoVerif, and then present the model itself: the security assumptions on the primitives, the model of the protocol, and the security properties that we want to prove. The complete CryptoVerif model, and the reusable library that provides the definitions of cryptographic primitives, can be found at https://cryptoverif.inria.fr/OEKE/.

\section{A. Review of CryptoVerif}

CryptoVerif builds proofs by sequences of games [43], [44]. It starts from the initial game given as input, which represents the protocol to prove in interaction with an adversary. Then, it transforms this game step by step using a set of predefined game transformations, such that each game is indistinguishable from the previous one.

More formally, a game $G$ interacts with an adversary represented by a context $C$, and we denote by $C[G]$ the combination of $C$ and $G$. During execution, $C[G]$ may execute events, collected in a sequence $\mathcal{E}$, and finally returns a result $a$, either a bitstring or the special value abort when the game has been aborted. These events and result can be used to distinguish games, so we introduce an additional algorithm, a distinguisher $D$ that takes as input the sequence of events $\mathcal{E}$ and the result $a$, and returns true or false. An example of distinguisher is $D_{e}$ defined by $D_{e}(\mathcal{E}, a)=$ true if and only if $e \in \mathcal{E}$ : this distinguisher detects the execution of event $e$. We will denote the distinguisher $D_{e}$ simply by $e$. More generally, distinguishers can detect various properties of the sequence of events $\mathcal{E}$ executed by the game and of its result $a$. We denote by $D \vee D^{\prime}, D \wedge D^{\prime}$, and $\neg D$ the distinguishers such that $\left(D \vee D^{\prime}\right)(\mathcal{E}, a)=D(\mathcal{E}, a) \vee D^{\prime}(\mathcal{E}, a)$, $\left(D \wedge D^{\prime}\right)(\mathcal{E}, a)=D(\mathcal{E}, a) \wedge D^{\prime}(\mathcal{E}, a)$, and $(\neg D)(\mathcal{E}, a)=$ $\neg D(\mathcal{E}, a)$, where $\vee$ is the logical disjunction, $\wedge$ the logical conjunction, and $\neg$ the logical negation. We denote by $\operatorname{Pr}[C[G]: D]$ the probability that $C[G]$ executes a sequence of events $\mathcal{E}$ and returns a result $a$, such that $D(\mathcal{E}, a)=$ true.
A context $C$ is acceptable for $G$ with public variables $V$ when it can read directly the variables of $G$ that are in $V$, and it makes no other access to variables of $G$. (This is more formally defined in Appendix A.) We define indistinguishability as an equivalence $G \approx_{p}^{V} G^{\prime}$ :

Definition 1 (Indistinguishability) We write $G \approx_{p}^{V} G^{\prime}$ when, for all contexts $C$ acceptable for $G$ and $G^{\prime}$ with public variables $V$ and all distinguishers $D$ that run in time at most $t_{D},\left|\operatorname{Pr}[C[G]: D]-\operatorname{Pr}\left[C\left[G^{\prime}\right]: D\right]\right| \leq p\left(C, t_{D}\right)$.

This definition formalizes that the probability that algorithms $C$ and $D$ distinguish the games $G$ and $G^{\prime}$ is at most $p\left(C, t_{D}\right)$. The probability $p$ typically depends on the runtime of $C$ and $D$, but may also depend on other parameters, such as the number of queries to each oracle made by $C$. That is why $p$ takes as arguments the whole algorithm $C$ and the runtime of $D$. When $V$ is empty, we write $G \approx_{p} G^{\prime}$. Therefore, we obtain a sequence of indistinguishable games $G_{0} \approx_{p_{1}}^{V} G_{1} \approx_{p_{2}}^{V} G_{2} \ldots G_{n-1} \approx_{p_{n}}^{V} G_{n}$, which implies $G_{0} \approx_{p_{1}+\ldots+p_{n}}^{V} G_{n}$. In the last game $G_{n}$, the desired security property is proved by direct inspection of the game, without using any computational assumption. For example, to bound the probability that an event $e$ is executed, event $e$ does not occur at all in the last game, so $\operatorname{Pr}\left[C\left[G_{n}\right]: e\right]=0$, hence the probability of executing $e$ in the initial game is $\operatorname{Pr}\left[C\left[G_{0}\right]: e\right] \leq\left(p_{1}+\cdots+p_{n}\right)\left(C, t_{e}\right)$.

The game transformations used by CryptoVerif can be split into two categories:

- syntactic transformations, which are used by CryptoVerif to simplify games and to prepare cryptographic transformations. These transformations do not rely on any security assumption on primitives.

- cryptographic transformations, which rely on a security assumption on a primitive. These security assumptions are themselves formalized as indistinguishability properties $L \approx_{p} R$, which are given as input to CryptoVerif and need to be proved manually. They are proved once for each primitive and can then be reused in many protocols. We present such equivalences for the primitives used in OEKE below.

CryptoVerif uses these equivalences to perform proofs by reduction automatically. It detects that a game $G$ can be written as a context $C$ that calls the oracles of $L$, that is, $G \approx_{0}^{V} C[L]$ by purely syntactic transformations, and builds a game $G^{\prime}$ such that $C[R] \approx_{0}^{V} G^{\prime}$ by purely syntactic transformations. $C$ is the simulator usually defined for reductions. From $L \approx_{p} R$, we can infer that $C[L] \approx_{p^{\prime}}^{V} C[R]$ where $V$ is a subset of the variables of $C$ and $p^{\prime}\left(C^{\prime}, t_{D}\right)=p\left(C^{\prime}[C[]], t_{D}\right)$. Indeed, if $C^{\prime}$ is the adversary against $C[L] \approx{ }_{p^{\prime}}^{V} C[R]$, the adversary against $L \approx_{p} R$ is $C^{\prime}[C[]]$. Therefore, $G \approx_{p^{\prime}}^{V} G^{\prime}$ and CryptoVerif can transform $G$ into $G^{\prime}$. 


$$
\begin{aligned}
& \hline L_{1}=\text { foreach } i h \leq n h \text { do } k \stackrel{R}{\leftarrow} \text { key; } \\
& \quad(\text { foreach } i \leq n \text { do } \\
& \text { OH }(x: \text { hashinput }):=\operatorname{return}(\operatorname{hash}(k, x)) \mid \\
& \text { foreach } \text { ieq } \leq \text { neq do } \\
& \text { Oeq }\left(x^{\prime}: \text { hashinput, } r^{\prime}: \text { hashoutput }\right):= \\
& \text { return } \left.\left(r^{\prime}=\operatorname{hash}\left(k, x^{\prime}\right)\right)\right)
\end{aligned}
$$

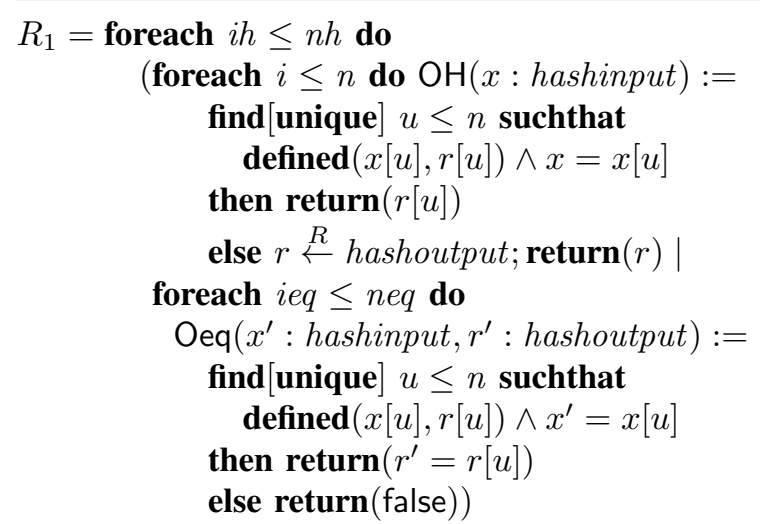

Figure 2. Random oracle model

\section{B. The Random Oracle Model}

The random oracle model was introduced in [60] to model hash functions. It was encoded in CryptoVerif in [50]. We improve this model by using the equivalence $L_{1} \approx_{\text {\#Oeq//hashoutput } \mid} R_{1}$ where $L_{1}$ and $R_{1}$ are defined in Figure 2. This model is not specific to OEKE. The hash function hash takes as input a key of type key and the bitstring to hash of type hashinput and returns a result of type hashoutput. The key models the choice of the hash function. The key must be chosen once and for all at the beginning of the game for each hash function, and the game must include a hash oracle, which allows the adversary to compute hashes. For each hash function indexed by $i h \leq n h$, the games $L_{1}$ and $R_{1}$ define two oracles, $\mathrm{OH}$ and Oeq:

- In $L_{1}, \mathrm{OH}(x)$ returns the image of $x$ by hash $(k, \cdot)$. This oracle can be called at most $n$ times for each hash function, and its calls are indexed by $i \in[1, n]$, as defined by foreach $i \leq n$ do. We can replace this oracle with a random oracle, that is, an oracle that returns a fresh random number when it is called with a new argument, and the previously returned result when it is called with the same argument as in a previous call. Such a random oracle is implemented in $R_{1}$ as follows. Like all variables defined under foreach $i \leq n, x$ is in fact an array indexed by $i$, so that $x[u]$ represents the value of $x$ in the $u$-th call to $\mathrm{OH}$. The find construct looks for an index $u$ such that $x[u]$ and $r[u]$ are defined, and $x=x[u]$, that is, the current argument of $\mathrm{OH}$ is the same as the argument in the $u$-th call, and if we find one, then we return the result of the $u$-th call, $r[u]$.
Otherwise, we return a fresh random number $r$.

- The oracle Oeq aims to optimize the treatment of comparisons with the result of the hash function, an operation that appears frequently. This oracle appears only when the output type of the random oracle, hashoutput, is large enough so that the probability of collision between a random element of hashoutput and a constant is small.

In $L_{1}$, the oracle $\operatorname{Oeq}\left(x^{\prime}, r^{\prime}\right)$ compares $r^{\prime}$ with hash $\left(k, x^{\prime}\right)$. In $R_{1}$, this comparison is replaced with a lookup in previous calls to the hash function. If $x^{\prime}$ was already given as argument to hash $(k, \cdot)$, in the $u$-th call $\left(x^{\prime}=x[u]\right)$, then hash $\left(k, x^{\prime}\right)$ is $r[u]$, so we compare $r^{\prime}$ with $r[u]$. Otherwise, $x^{\prime}$ was never given as argument to $\operatorname{hash}(k, \cdot)$, so hash $\left(k, x^{\prime}\right)$ is a fresh random number, and it is equal to $r^{\prime}$ with probability $1 / \mid$ hashoutput $\mid$. We eliminate this case in $R_{1}$, so the result of the comparison $r^{\prime}=\operatorname{hash}\left(k, x^{\prime}\right)$ is replaced with false and the probability of distinguishing $L_{1}$ from $R_{1}$ is at most \#Oeq/|hashoutput $\mid$, where \#Oeq denotes the total number of calls to Oeq.

We can notice that there exists at most one $u$ that can satisfy the condition of find in $\mathrm{OH}$ in $R_{1}$. Indeed, suppose that $u_{1} \neq u_{2}$ are such that $x\left[u_{1}\right], r\left[u_{1}\right], x\left[u_{2}\right], r\left[u_{2}\right]$ are defined and $x=x\left[u_{1}\right]=x\left[u_{2}\right]$. Suppose that the query $\mathrm{OH}$ with $i=u_{1}$ is called before $\mathrm{OH}$ with $i=u_{2}$. (The other case is symmetric.) Thus, when executing the query $\mathrm{OH}$ with $i=u_{2}, x\left[u_{1}\right]$ and $r\left[u_{1}\right]$ are defined and $x\left[u_{2}\right]=x\left[u_{1}\right]$, so the find succeeds with $u=u_{1}$, so $r\left[u_{2}\right]$ will not be defined (since $r$ is defined only in the else branch of the find). Contradiction. Therefore, $u$ is unique. Following a similar reasoning, $u$ is also unique in Oeq in $R_{1}$. That is why the finds in $R_{1}$ are marked [unique]. Formally, the modifier [unique] means that, in case several choices satisfy the condition of find, an event NonUnique occurs and the game is aborted. As we have shown, the event NonUnique never occurs in $R_{1}$, so the modifier [unique] does not alter the equivalence $L_{1} \approx \#$ Oeq/|hashoutput $\mid R_{1}$. The modifier [unique] allows additional transformations of find, which are correct only when there never exist several choices that make the condition of the find succeed. These transformations are detailed in Appendix E-B.

The novelties with respect to [50] are the use of keyed hash functions, the oracle Oeq, and the modifier [unique]. We believe that using keyed hash functions leads to a better modeling of random oracles, for several reasons:

- In the random oracle model, the adversary cannot evaluate the hash function by himself, without calling the random oracle. With the key, this is natural, since the adversary does not have the key, whereas in the absence of key, this is counterintuitive: the adversary should be able to reproduce the algorithm of $h$.

- In the absence of key, the transformation of $L_{1}$ into $R_{1}$ 


$$
\begin{aligned}
& L_{2}=\text { foreach } i c k \leq n c k \text { do } c k \stackrel{R}{\leftarrow} \text { cipherkey; } \\
& \quad(\text { foreach } i e \leq n e \text { do } \operatorname{Oenc}(m e: \text { blocksize, } k e: k e y):=\operatorname{return}(\operatorname{enc}(c k, m e, k e)) \mid \\
& \quad \text { foreach } i d \leq n d \text { do } \operatorname{Odec}(m d: \text { blocksize, } k d: \text { key }):=\operatorname{return}(\operatorname{dec}(c k, m d, k d)))
\end{aligned}
$$

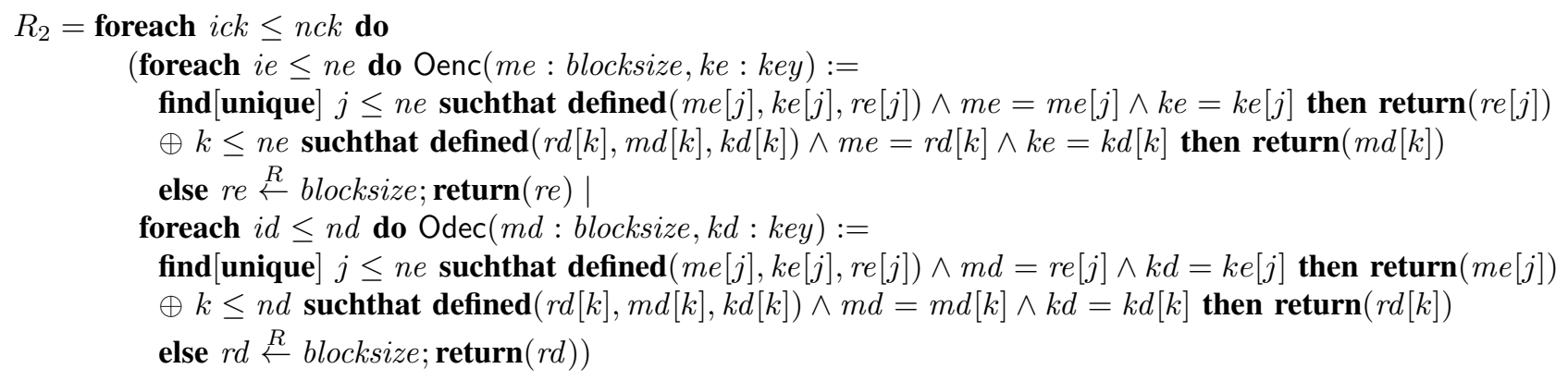

Figure 3. Ideal cipher model

above replaces a deterministic function $h$ with a probabilistic one, since the results are chosen randomly in the right-hand side. The key removes this discrepancy: with the key, the hash oracle is also probabilistic in the left-hand side thanks to the choice of the key.

- The transformation of $L_{1}$ into $R_{1}$ above is correct only when it is applied to all occurrences of $h$ simultaneously. In the absence of key, this has to be enforced by an additional constraint on the transformation. With the key, this is naturally enforced, since all occurrences of the key need to be encoded as calls to the oracles of $L_{1}$ for the transformation to be performed.

- Finally, keyed hash functions are used in the modeling of other assumptions on hash functions, such as collision resistance. By always using keyed hash functions, we can easily change the assumption on the hash function without changing its interface.

Designing CryptoVerif specifications of primitives requires some expertise. That is why the specifications for most common cryptographic primitives are grouped in a reusable library. Therefore, CryptoVerif users generally do not have to design such specifications.

\section{The Ideal Cipher Model}

The ideal cipher model [61] models block ciphers by saying that encryption and decryption are two random permutations, inverse of each other. This can be encoded in CryptoVerif similarly to the random oracle model: we replace encryption and decryption with lookups in previous encryption/decryption queries; if a previous query matches, we return the previous result; otherwise, we return a fresh random number. This is modeled by the equivalence $L_{2} \approx_{p_{2}}$ $R_{2}$ where $L_{2}$ and $R_{2}$ are defined in Figure 3 and $p_{2}=$ $(\#$ Oenc $+\#$ Odec $)(\#$ Oenc $+\#$ Odec -1$) / \mid$ blocksize $\mid$. The encryption and decryption functions map bitstrings of type blocksize to bitstrings of type blocksize; they take two keys as additional arguments: the standard encryption/decryption key of type key, but also a key of type cipherkey that models the choice of the scheme itself (like the key of the hash function in Section III-B). The games $L_{2}$ and $R_{2}$ define two oracles Oenc and Odec, respectively the encryption and decryption oracles. In $L_{2}$, they call the encryption and decryption functions. In $R_{2}$, they are replaced with lookups in previous encryption/decryption queries. For instance, for oracle Oenc, we look for a previous encryption query of the same cleartext ( $m e=m e[j]$ ) under the same key (ke $=k e[j]$ ) and, if we find one, we return the same ciphertext $r e[j]$. We also look for a previous decryption query that has returned as cleartext the cleartext to encrypt (me $=r d[k])$ using the same key $(k e=k d[k])$ and, if we find one, we return the corresponding ciphertext $m d[k]$. Otherwise, we return a fresh random ciphertext re. This definition does not yield random permutations, because the random choices of $r e$ and $r d$ may collide with each other and with previous values of $m e$ and $m d$. Let us consider a game $R_{2}^{\prime}$ obtained from $R_{2}$ by excluding such collisions. By adapting the reasoning used for the random oracle model in Section III-B, we can show that, in $R_{2}^{\prime}$, there never exist several choices of $j / k$ that satisfy the conditions of the finds in Oenc and Odec, so these finds can be marked [unique] without modifying their behavior. The game $L_{2}$ is perfectly indistinguishable from $R_{2}^{\prime}$, and $R_{2}^{\prime}$ can be distinguished from $R_{2}$ with probability at most $p_{2}$ (the probability of the collisions excluded in $R_{2}^{\prime}$ ), so the adversary can distinguish $L_{2}$ from $R_{2}$ with probability at most $p_{2}$.

As for the random oracle model, we can optimize the case of comparisons with the result of encryption or decryption, to avoid generating a fresh random value in this case. We omit this optimization in this paper for simplicity, as it is not necessary for proving the security of OEKE.

\section{The Computational Diffie-Hellman Assumption}

A classical intractability assumption in asymmetric cryptography is the hardness of the Diffie-Hellman problem: let 
us be given a group $\mathbb{G}$ of prime order $q$, with a generator $g$, and two random elements $A=g^{a}$ and $B=g^{b}$ with $a, b \in[1, q-1]$, compute $\mathrm{CDH}_{g}(A, B)=g^{a b}$. The Computational Diffie-Hellman $(\mathrm{CDH})$ assumption claims that for any polynomial-time adversary $\mathcal{A}, \operatorname{Succ}_{\mathbb{G}}^{\text {cdh }}(\mathcal{A})=$ $\operatorname{Pr}\left[\mathcal{A}(\mathbb{G}, g, A, B)=\mathrm{CDH}_{g}(A, B)\right]$ is negligible. More generally, we note $\operatorname{Succ}_{\mathbb{G}}^{\text {cdh }}(t)$ the maximal success probability for any adversary $\mathcal{A}$ within time $t$.

This assumption can be written in CryptoVerif as follows:

$$
\begin{aligned}
& \text { foreach } i \leq n \text { do } a \stackrel{R}{\leftarrow} Z ; b \stackrel{R}{\leftarrow} Z ; \\
& \quad(\mathrm{OA}():=\exp (\mathrm{g}, a)|\mathrm{OB}():=\exp (\mathrm{g}, b)| \\
& \quad \text { foreach } i^{\prime} \leq n^{\prime} \text { do } \operatorname{ODDH}(z: G):= \\
& z=\exp (\mathrm{g}, \operatorname{mult}(a, b))) \\
& \approx_{\# \mathrm{ODDH} \times \operatorname{Succ}_{\mathbb{G}}^{\text {cdh }}\left(t+(n+\# \mathrm{ODDH}) \tau_{\text {exp }}\right)} \\
& \text { foreach } i \leq n \text { do } a \stackrel{R}{\leftarrow} Z ; b \stackrel{R}{\leftarrow} Z ; \\
& \quad(\mathrm{OA}():=\exp (\mathrm{g}, a)|\mathrm{OB}():=\exp (\mathrm{g}, b)|
\end{aligned}
$$$$
\text { foreach } \left.i^{\prime} \leq n^{\prime} \text { do } \operatorname{ODDH}(z: G):=\text { false }\right)
$$

The type $Z$ represents $[1, q-1]$ modulo $q$, that is, the group $\mathbb{Z}_{q}^{*}$; mult is the product in that group; $G$ represents the group $\mathbb{G}$ without its neutral element; and exp is the exponentiation $G \times Z \rightarrow G$. These two games define three oracles: OA and $\mathrm{OB}$ return the exponentials $g^{a}$ and $g^{b}$ respectively, and the oracle ODDH checks whether its argument $z$ is equal to $g^{a b}$ in the left-hand side while it always returns false in the right-hand side. The adversary can distinguish these two games if and only if it can provide a $z$ such that $z=g^{a b}$, that is, it breaks the CDH assumption. However, in CryptoVerif, this model requires that $a$ and $b$ be chosen one after the other under the same foreach: while this is true in some cryptographic schemes such as ElGamal, this is not true for most protocols: as in OEKE, $a$ and $b$ are chosen by different protocol participants that can each execute several sessions.

Therefore, we need a more general model, which is given by the indistinguishability between the two games presented in Figure 4. In these two games, one generates $n a$ exponents $a, n b$ exponents $b$ and the adversary (any context) has access to various oracles: $O A$ and $O B$ that return the group elements associated to $a$, resp. $b$; Oa and $\mathrm{Ob}$ that return the exponents $a$ and $b$ themselves; ODHa and $\mathrm{ODHb}$ that map an exponent $x$ to $g^{a x}$, resp. $g^{b x}$ (these oracles can also be computed by raising OA, resp. $\mathrm{OB}$ to the power $x$; however, mentioning them explicitly in the definition of $\mathrm{CDH}$ is useful because CryptoVerif rewrites $\exp (\exp (\mathrm{g}, a), x)$ into $\exp (\mathrm{g}, \operatorname{mult}(a, x))$ by (1) defined below and does not recognize that the latter can be computed from OA); and Diffie-Hellman decisions oracles ODDHa and ODDHb that check whether the adversary correctly solved a Diffie-Hellman problem with the above generated elements. Basically, the difference between the two games is in the answers of the decision oracles: in the first game they answer correctly, while in the second game, they answer false if the adversary did not ask for
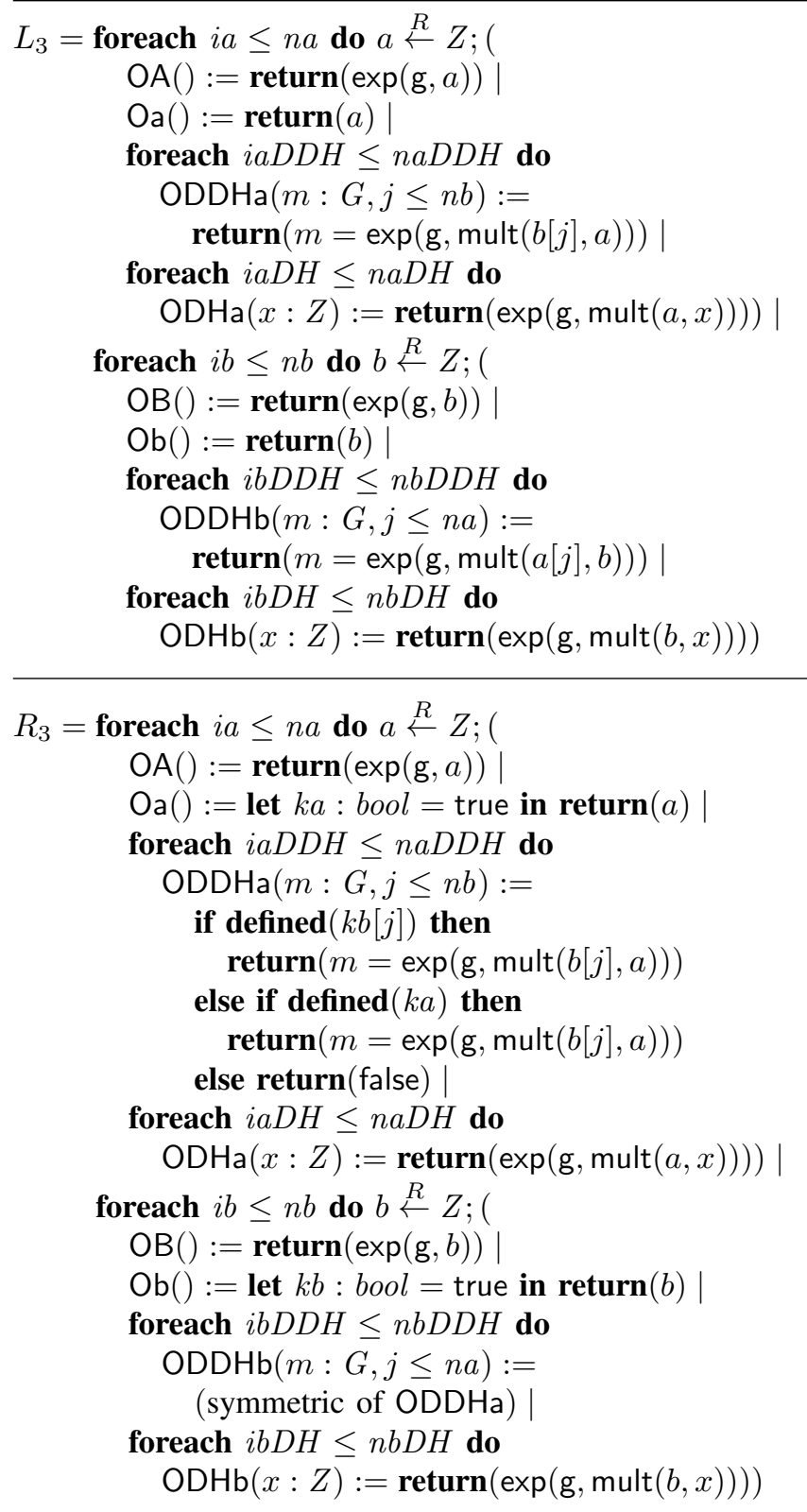

Figure 4. Computational Diffie-Hellman assumption

any of the two exponents. Unless the adversary can break the Diffie-Hellman problem, and then ask correct DiffieHellman decision queries, the two executions are perfectly indistinguishable. In more detail, in $R_{3}$, the variable $k a$ is defined if and only if the oracle $\mathrm{Oa}$ has been called and thus the exponent $a$ has been asked by the adversary. All variables and oracles defined under foreach $i a \leq n a$ are implicitly indexed by $i a$, so that $k a[i a]$ is defined if and only if $a[i a]$ has been asked by the adversary. The variable $k b$ plays the same role for $b$. The oracle ODDHa computes the equality test $m=g^{a[i a] b[j]}$ when $b[j]$ has been asked by the adversary, i.e., $k b[j]$ is defined, or $a[i a]$ has been asked 
by the adversary, i.e. $k a[i a]$ (abbreviated $k a$ ) is defined. Otherwise, it returns false.

In Appendix B-A, we prove that $L_{3} \approx_{p_{3}} R_{3}$, that is, no adversary can distinguish the two games $L_{3}$ and $R_{3}$, within time $t$, with advantage greater than

$p_{3}=(\# \mathrm{ODDHa}+\# \mathrm{ODDHb})(3 \# \mathrm{Oa}+1)(3 \# \mathrm{Ob}+1) \times$

$$
\operatorname{Succ}_{\mathbb{G}}^{\text {cdh }}\left(t+\left(n a+n b+q_{\text {ddh }}^{\prime}+\# \mathrm{ODHa}+\# \mathrm{ODHb}\right) \tau_{\exp }\right) \text {. }
$$

where $q_{\mathrm{ddh}}^{\prime}=0$ if $\mathrm{Oa}$ and $\mathrm{Ob}$ are never called ( $\# \mathrm{Oa}=0$ and $\# \mathrm{Ob}=0)$ and $q_{\mathrm{ddh}}^{\prime}=\# \mathrm{ODDHa}+\# \mathrm{ODDHb}$ otherwise. The proof technique consists in guessing the two elements $a$ and $b$ that will be involved in the critical decisional DiffieHellman query (but with Coron's improvement [62]), and then to guess the critical query, hence the factor \#ODDHa+ \#ODDHb.

For this equivalence to be supported by CryptoVerif, we had to implement two extensions:

- Oracles ODDHa and ODDHb take as argument an array index $j$, which was not supported.

- In typical usages of the CDH assumption in protocols, $g^{a b}$ is often an argument of a hash function in the random oracle model. The transformation that comes from the random oracle model, presented in Section III-B, transforms hash $\left(\ldots g^{a b} \ldots\right)$ into lookups that compare $g^{a b}$ with previous arguments of hash. These comparisons $m=g^{a b}$, which occur in conditions of find, are themselves transformed into find using the $\mathrm{CDH}$ assumption. We therefore end up with a find inside the condition of a find, which was not supported.

Moreover, in $R_{3}$, we actually use the symbol exp' instead of exp, although the two symbols represent the same function on bitstrings. This technique avoids infinite loops: if we used exp in $R_{3}, R_{3}$ would be an instance of $L_{3}$, so the transformation of $L_{3}$ into $R_{3}$ could be applied again on $R_{3}$, leading to an infinite loop. By using exp', we prevent applying the transformation again on occurrences that have already been transformed.

In addition to the modeling of the $\mathrm{CDH}$ assumption itself, our model includes properties that hold in any group $\mathbb{G}$ of prime order $q$, where $Z$ is $\mathbb{Z}_{q}^{*}$, mult is the product in $\mathbb{Z}_{q}^{*}$, $G$ is the group $\mathbb{G}$ without its neutral element, and $\exp$ is exponentiation.

The multiplication mult is commutative and we have the following equalities:

$$
\begin{aligned}
& \forall X: G, \forall x: Z, \forall y: Z \\
& \quad \exp (\exp (X, x), y)=\exp (X, \operatorname{mult}(x, y)) \\
& \forall X: G, \forall X^{\prime}: G, \forall x: Z \\
& \quad\left(\exp (X, x)=\exp \left(X^{\prime}, x\right)\right)=\left(X=X^{\prime}\right) \\
& \forall X: G, \forall x^{\prime}: Z, \forall x: Z ; \\
& \quad\left(\exp (X, x)=\exp \left(\mathrm{g}, \operatorname{mult}\left(x^{\prime}, x\right)\right)\right)=\left(X=\exp \left(\mathrm{g}, x^{\prime}\right)\right)
\end{aligned}
$$

$$
\begin{aligned}
& \forall X: G, \forall x: Z, \forall y: Z ; \\
& \quad(\exp (X, x)=\exp (X, y))=(x=y) \\
& \forall x: Z, \forall y: Z, \forall y^{\prime}: Z ; \\
& \quad\left(\operatorname{mult}(x, y)=\operatorname{mult}\left(x, y^{\prime}\right)\right)=\left(y=y^{\prime}\right)
\end{aligned}
$$

The commutativity of mult combined with (1) shows that $\left(g^{a}\right)^{b}=g^{a b}=g^{b a}=\left(g^{b}\right)^{a}$, the standard equality that shows that the client and the server compute the same key in the Diffie-Hellman key exchange. Equations (2), (3), and (4) express the injectivity of exp. Equation (2) is obtained by raising $X^{x}=X^{\prime x}$ to the power $x^{-1}$ ( $x$ is invertible modulo $q$ because $x \in \mathbb{Z}_{q}^{*}$ ). Equation (3) is a particular case of (2), with $X^{\prime}=g^{x^{\prime}}$ (but CryptoVerif would not infer (3) from (2)). Equation (4) holds because $X$ is a generator of the group $\mathbb{G}$ of prime order $q(X \in G$ is not the neutral element) and $x, y \in \mathbb{Z}_{q}^{*}$. We have similar equations for $\exp ^{\prime}$. Equation (5) is obtained by dividing the equality $x y=x y^{\prime}$ by $x$ in the group $\mathbb{Z}_{q}^{*}$.

If we choose uniformly $x$ in $Z$ and compute $g^{x}$, the result is a uniformly distributed element of $G$, so we have the equivalence

$$
\begin{aligned}
\text { foreach } i \leq n \text { do } x \stackrel{R}{\leftarrow} Z ; \mathrm{OX}():=\operatorname{return}(\exp (\mathrm{g}, x)) \\
\approx_{0} \text { foreach } i \leq n \text { do } X \stackrel{R}{\leftarrow} G ; \mathrm{OX}():=\operatorname{return}(X) .
\end{aligned}
$$

We have a similar equivalence for $\exp ^{\prime}$. Although equivalences are symmetric, CryptoVerif always applies them from left to right, replacing the code of oracles in the left-hand side with the corresponding code in the right-hand side. For this reason, we state the symmetric equivalence explicitly:

$$
\begin{aligned}
& \text { foreach } i \leq n \text { do } X \stackrel{R}{\leftarrow} G ; \mathrm{OX}():=\operatorname{return}(X) \\
& \quad \approx_{0}[\text { manual }]
\end{aligned}
$$

foreach $i \leq n$ do $x \stackrel{R}{\leftarrow} Z ; \mathrm{OX}():=\operatorname{return}(\exp (\mathrm{g}, x))$.

However, this equivalence is applied only manually, as indicated by [manual]; otherwise, it would yield an infinite loop by applying alternatively (6) and (7). We use the following more restricted form for automatic proofs

$$
\begin{aligned}
& \text { foreach } i \leq n \text { do } X \stackrel{R}{\leftarrow} G \\
& (\mathrm{OX}():=\operatorname{return}(X) \mid \\
& \quad \text { foreach } i^{\prime} \leq n^{\prime} \operatorname{do} \operatorname{OXm}(m: Z) \\
& {[\text { useful_change] }:=\operatorname{return}(\exp (X, m)))} \\
& \approx_{0} \text { foreach } i \leq n \text { do } x \stackrel{R}{\leftarrow} Z \\
& (\mathrm{OX}():=\operatorname{return}(\exp (\mathrm{g}, x)) \mid \\
& \quad \text { foreach } i^{\prime} \leq n^{\prime} \text { do } \mathrm{OXm}(m: Z):= \\
& \quad \text { return }(\exp (\mathrm{g}, \operatorname{mult}(x, m)))) .
\end{aligned}
$$

which can be applied only when $X$ is used as argument of exp. (The annotation [useful_change] requires that oracle 
OXm is called at least once for CryptoVerif to apply this equivalence.)

Additional properties are defined in Appendix B-B. We stress that our above modeling is not specific to the OEKE protocol. We have also used it to prove a signed DiffieHellman key exchange, and we believe that it can be used for proving many other protocols.

\section{E. The Protocol Itself}

If we consider a general configuration with several clients and servers, each client-server pair shares a different password, and there is no other secret shared initially. Therefore, different client-server pairs have no common secret, so we can encode a single client $U$ and a single server $S$ that wish to talk to each other; the other clients and servers, which may be corrupted, and the interactions of $U$ and $S$ with other clients and servers are included in the adversary. This model supports static corruptions; dynamic corruptions and forward secrecy properties are left for future work.

The protocol model first chooses random keys $h k 0$ and $h k 1$ to model the choice of the hash functions h0 (i.e. $\mathcal{H}_{0}$ ) and h1 (i.e. $\mathcal{H}_{1}$ ) respectively and a key $c k$ to model the choice of the ideal cipher scheme. It also randomly chooses a password $p w 0$ in the type passwd. Then, it makes available hash oracles for h0 and h1, encryption and decryption oracles, as well as oracles that represent the client and the server. As an example, we detail the code for the client:

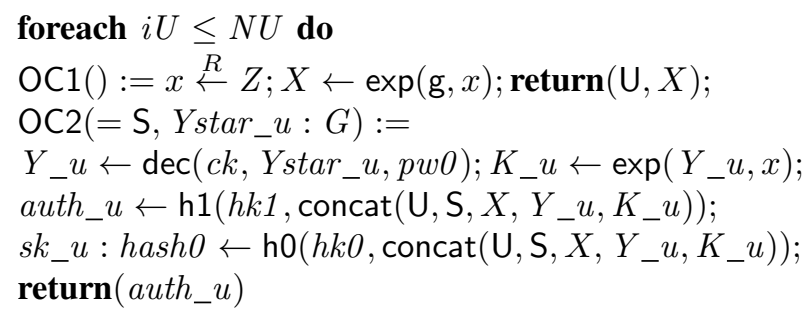

This code models $N U$ sessions of the client, indexed by $i U$. Each session defines two oracles OC1 and OC2. OC1 takes no argument and returns the first message of the protocol $\mathrm{U}, X$ computed as specified in Figure 1. OC2 takes as argument the second message of the protocol S, Ystar_ $u$ received by the client and returns the third one auth_u. It also computes the shared key $s k \_u$. In this code, concat $\left(\mathrm{U}, \mathrm{S}, X, Y_{-} u, K_{-} u\right)$ is the concatenation $\mathrm{U}\|\mathrm{S}\| X\left\|Y_{\_} u\right\| K_{-} u$. These oracles are implicitly indexed by $i U$, so that they can be written OC1 $[i U]$, OC2 $[i U]$. (This index is omitted in CryptoVerif code for readability.) The adversary can call the oracles with any index it likes in the order it likes, except that, obviously, OC2 $[i U]$ can be called only if $\mathrm{OC} 1[i U]$ has been called before with the same $i U$. This gives the adversary full control over the network.

We represent $N S$ sessions of the server in a similar way. The $N U$ sessions of the client and the $N S$ sessions of the server model active attacks. Additionally, we represent $N P$ sessions of the protocol in which the adversary just eavesdrops messages without altering them. In order to represent such sessions, we simply compute and output their transcript. They model passive attacks. Since we are considering dictionary attacks against a password-authenticated key exchange protocol, it is important to distinguish passive sessions/attacks from active ones against the honest players.

\section{F. Security Properties}

Our goal is to prove that OEKE is a secure key exchange that provides unilateral (explicit) authentication. (OEKE guarantees client authentication but not server authentication.) To do that, we follow the ideas of [63, Section 7.2] and [64]: instead of proving semantic security of the key and authentication, we prove secrecy of the key on the client side and a slightly stronger authentication property. This technique avoids the burden of considering partnering when proving secrecy of the key and still implies authenticated key exchange [63, Proposition 4]: intuitively, authentication guarantees that a key of the server is also a key of a client. Authentication is modeled by correspondence properties [65] of the form "if some event occurs, then some other event occurred". There are still three differences with respect to [63]:

- [63] considers mutual authentication, while we consider unilateral authentication, so we remove the correspondence that guaranteed authentication of the server.

- In [63], each protocol participant may interact with honest participants ( $U$ and $S$ here) but also with dishonest participants, and in the latter situation, the exchanged key is published when the participant accepts. As mentioned in Section III-E, in OEKE, we need not code explicitly for $U$ and $S$ interacting with other clients and servers, so the output of the exchanged key disappears.

- As in [64], we require that there is at most one session of the client with a given transcript, instead of requiring that the client and server have the same key when they have the same transcript as in [63]. Together with unilateral authentication, the former strictly implies the latter.

Taking into account these points, we add events to record that the participants accept or terminate:

- event $\operatorname{acceptU}\left(\mathrm{U}, X, \mathrm{~S}, Y s t a r_{-} u, a u t h \_u, s k_{-} u, i U\right)$ when the client accepts (line "accept $\leftarrow$ true" of the client in Figure 1, that is, before the last line in the code of Section III-E).

- event termS $\left(\mathrm{U}, X_{-} \_, \mathrm{S}, Y\right.$ star, auth_s, sk_s) when the server terminates (line "terminate $\leftarrow$ true" of the server in Figure 1).

and we prove that the resulting process preserves the secrecy of $s k_{-} u$ and satisfies the correspondences

$$
\begin{aligned}
& \text { inj-event }(\operatorname{termS}(U, X, S, Y \text { star }, a, k)) \Rightarrow \\
& \quad \text { inj-event }(\operatorname{acceptU}(U, X, S, Y \text { star }, a, k, u))
\end{aligned}
$$




$$
\begin{aligned}
& \operatorname{event}(\operatorname{acceptU}(U, X, S, Y \text { star, } a, k, u)) \wedge \\
& \quad \operatorname{event}\left(\operatorname{acceptU}\left(U, X, S, Y \text { star }, a, k^{\prime}, u^{\prime}\right)\right) \Rightarrow u=u^{\prime}
\end{aligned}
$$

with public variables $\left\{s k \_u\right\}$. A variant of [63, Proposition 4] allows us to conclude one-way authenticated key exchange. Next, we define secrecy and correspondences.

Intuitively, the secrecy of $s k \_u$ means that the keys $s k \_u$ of all sessions of the client are indistinguishable from independent random keys. Formally, secrecy is defined as follows:

Definition 2 (Secrecy) Assume that the variable $x$ of type $T$ is defined in $G$ under a single foreach $i \leq n$. The game $G$ preserves the secrecy of $x$ up to probability $p$ when, for all contexts $C$ acceptable for $G \mid R_{x}$ without public variables that do not contain $\mathrm{S}$ and $\overline{\mathrm{S}}, \operatorname{Pr}\left[C\left[G \mid R_{x}\right]: \mathrm{S}\right]-\operatorname{Pr}[C[G \mid$ $\left.\left.R_{x}\right]: \overline{\mathrm{S}}\right] \leq p(C)$ where

$$
\begin{gathered}
R_{x}=O_{0}():=b \stackrel{R}{\leftarrow} \text { bool; return; } \\
\left(\text { foreach } i^{\prime} \leq n^{\prime} \text { do } O(u:[1, n]):=\right. \\
\text { if defined }(x[u]) \text { then } \\
\text { if } b \text { then return }(x[u]) \text { else } \\
\text { find } u^{\prime} \leq n^{\prime} \text { suchthat defined }\left(y\left[u^{\prime}\right], u\left[u^{\prime}\right]\right) \wedge \\
u\left[u^{\prime}\right]=u \text { then return }\left(y\left[u^{\prime}\right]\right) \text { else } \\
y \stackrel{R}{\leftarrow} T ; \text { return }(y) \\
\mid O^{\prime}\left(b^{\prime}: b o o l\right):=\text { if } b=b^{\prime} \text { then event_abort } \mathrm{S} \\
\text { else event_abort } \overline{\mathrm{S}})
\end{gathered}
$$

$O_{0}, O, O^{\prime}, b, b^{\prime}, u, u^{\prime}, y, \mathrm{~S}$, and $\overline{\mathrm{S}}$ do not occur in $G$.

We define the secrecy of $x$ with the Real-or-Random model of [66]: in $R_{x}$, we choose a random bit $b$, and provide the oracle $O$ that the adversary can use to perform several test queries on $x[u]$ : if $b=1$, the test query returns $x[u]$; if $b=0$, it returns a random value $y$ (the same value if the same query $x[u]$ is asked twice). Finally, the adversary should guess the bit $b$ : it calls oracle $O^{\prime}$ with its guess $b^{\prime}$ and, if the guess is correct, then event $S$ is executed, and otherwise, event $\bar{S}$ is executed. The probability of getting some information on the secret is the difference between the probability of $S$ and the probability of $\overline{\mathrm{S}}$. (When the game always runs oracle $O^{\prime}$, we have $\operatorname{Pr}\left[C\left[G \mid R_{x}\right]: \overline{\mathrm{S}}\right]=1-\operatorname{Pr}\left[C\left[G \mid R_{x}\right]: \mathrm{S}\right]$, so the advantage of the adversary is $\operatorname{Pr}\left[C\left[G \mid R_{x}\right]: \mathrm{S}\right]-$ $\operatorname{Pr}\left[C\left[G \mid R_{x}\right]: \overline{\mathrm{S}}\right]=2 \operatorname{Pr}\left[C\left[G \mid R_{x}\right]: \mathrm{S}\right]-1$, which is a more standard formula.) As shown in [66], the Real-orRandom model is stronger than the Find-Then-Guess model used in [55], which allows a single test query and several reveal queries. (Reveal queries always return the real $x[u]$.)

The correspondence (9) means that each execution of event termS $(U, X, S, Y$ star, $a, k)$ corresponds to a distinct execution of event acceptU $(U, X, S, Y$ star, $a, k, u)$; in other words, each session of the server that accepts with transcript $U, X, S, Y$ star, $a$ and shared key $k$ corresponds to a distinct session of the client that accepts with the same transcript and same key. It corresponds to the authentication of the client. The keyword inj-event is used in CryptoVerif to require injective correspondences, that is, acceptU has been executed at least as many times as termS, and not only once. The correspondence (10) means that when events acceptU $(U, X, S, Y$ star, $a, k, u)$ and acceptU $\left(U, X, S, Y\right.$ star $\left., a, k^{\prime}, u^{\prime}\right)$ have been executed, $u=u^{\prime}$, that is, if two executions of a client have the same transcript, then they have the same replication index $u=u^{\prime}$, so they are in fact the same execution. In other words, there is a single execution of the client with a given transcript. These correspondences are proved "with public variables $\left\{s k_{-} u\right\}$ ", that is, they hold even when the adversary is allowed to access $s k \_u$ directly. Formally, we write $\mathcal{E} \vdash \psi \Rightarrow \varphi$ when the sequence of events $\mathcal{E}$ satisfies the correspondence $\psi \Rightarrow \varphi$. (This is formally defined in [63].) For instance, $\mathcal{E} \vdash$ inj-event $(\operatorname{termS}(U, X, S, Y$ star, $a, k)) \Rightarrow$ inj-event $(\operatorname{acceptU}(U, X, S, Y$ star $, a, k, u))$ if and only if, for each event $\operatorname{acceptU}(\ldots)$ in $\mathcal{E}$, there is a distinct event termS $(.$.$) in \mathcal{E}$ with the same arguments as the event acceptU $(. .$.$) (except that the last argument of acceptU does$ not appear in termS).

Definition 3 (Correspondence) The game $G$ satisfies the correspondence $\psi \Rightarrow \varphi$ with public variables $V$ up to probability $p$ if and only if, for all contexts $C$ acceptable for $G$ with public variables $V$ that do not contain events, $\operatorname{Pr}[C[Q]: D] \leq p(C)$, where $D(\mathcal{E}, a)=(\mathcal{E} \forall \forall \psi \Rightarrow \varphi)$.

\section{PROVING OEKE IN CRYPTOVERIF}

In the previous section, we have presented the formalization of the protocol given as input to CryptoVerif. In this section, we explain how CryptoVerif proceeds with the proof. The proof is guided by the user. The commands for guiding CryptoVerif can be given interactively, which allows one to see the current game and understand what should be done next, or in a proof $\{\ldots\}$ declaration in the CryptoVerif input file, so that CryptoVerif can then run on its own. The input file presented at https://cryptoverif.inria.fr/OEKE/ includes such a declaration. We stress that, even with manual guidance, all game transformations are verified by CryptoVerif, so that one cannot perform an incorrect proof.

\section{A. Limiting the Probability of Eliminated Collisions}

To improve the probability computations performed by CryptoVerif, the user can give an estimate of the number of allowed calls to oracles. This estimate can be

- small, for calls in which the adversary actively interacts with the honest participants and mounts detectable attacks, when these participants stop after a certain number of failed attempts. $N U$ and $N S$ are small in OEKE.

- passive (the default), for calls in which either the adversary passively listens to messages without modifying 
them ( $N P$ in OEKE), or the protocol does not limit the number of calls even in the case of incorrect messages.

- noninteractive, for calls that can be implemented by the adversary without actually interacting with honest participants. This is the case of calls to random oracles ( $q H 0$ calls to h0 and $q H 1$ calls to h1 in OEKE) as well as encryption and decryption oracles in the ideal cipher model ( $q E, q D$ in OEKE).

Numbers of calls annotated noninteractive are larger than those annotated passive, which are larger than those annotated small.

Similarly, estimates can also be given for the size of types:

- large means that the type is large enough to eliminate collisions among random values of that type automatically.

- password is for the type passwords (passwd), which is not so large, but for which we shall eliminate some collisions.

By default, CryptoVerif eliminates all collisions among random values of large types. To obtain a better probability bound, we tell CryptoVerif to eliminate collisions only when their probability is bounded by $n^{2} /|T|$ where $n$ is a number of calls of estimate at most noninteractive and $T$ is a type with size estimate at least large. This is done by the command allowed_collisions noninteractive ${ }^{\wedge} 2 /$ large.

\section{B. Proof of (10)}

The command success tells CryptoVerif to try proving all desired security properties. At this point, it succeeds in proving (10), but not the other properties, so we continue the proof.

\section{Applying Shoup's Lemma}

We introduce the events Auth and Encrypt, which correspond to cases in which the adversary succeeds in testing a password and were also used in the manual proof of [55]. We also introduce an event cdh, which corresponds to cases in which the adversary would break the computational DiffieHellman assumption.

By Shoup's lemma [43], if $G^{\prime}$ is obtained from $G$ by inserting an event $e$ and modifying the code executed after $e$, the probability of distinguishing $G^{\prime}$ from $G$ is bounded by the probability of executing $e$ : for all contexts $C$ acceptable for $G$ and $G^{\prime}$ (with any public variables) and all distinguishers $D,\left|\operatorname{Pr}[C[G]: D]-\operatorname{Pr}\left[C\left[G^{\prime}\right]: D\right]\right| \leq \operatorname{Pr}\left[C\left[G^{\prime}\right]: e\right]$. Hence, $\operatorname{Pr}[C[G]: D] \leq \operatorname{Pr}\left[C\left[G^{\prime}\right]: e\right]+\operatorname{Pr}\left[C\left[G^{\prime}\right]: D\right]$. We improve over this computation of probabilities by considering $e$ and $D$ simultaneously instead of making the sum of the two probabilities: $\operatorname{Pr}[C[G]: D] \leq \operatorname{Pr}\left[C\left[G^{\prime}\right]: D \vee e\right]$.

Lemma 1 Let $C$ be a context acceptable for $G$ and $G^{\prime}$ with public variables $V$.

1) If $G^{\prime}$ differs from $G$ only when $G^{\prime}$ executes event e, then $\operatorname{Pr}[C[G]: D] \leq \operatorname{Pr}\left[C\left[G^{\prime}\right]: D \vee e\right]$.
2) If $G$ differs from $G^{\prime}$ only when $G$ executes event NonUnique and $D=\left(D_{0} \wedge \neg\right.$ NonUnique $) \vee e_{1} \vee \ldots \vee e_{n}$ where we abort just after executing events $e_{1}, \ldots, e_{n}$, then $\operatorname{Pr}[C[G]: D] \leq \operatorname{Pr}\left[C\left[G^{\prime}\right]: D\right]$.

3) If $G \approx_{p}^{V} G^{\prime}$, then $\operatorname{Pr}[C[G]: D] \leq p\left(C, t_{D}\right)+$ $\operatorname{Pr}\left[C\left[G^{\prime}\right]: D\right]$.

4) $\operatorname{Pr}\left[C[G]: D \vee D^{\prime}\right] \leq \operatorname{Pr}[C[G]: D]+\operatorname{Pr}\left[C[G]: D^{\prime}\right]$.

This lemma, and Lemma 2 below, are proved in Appendix C. In order to bound the probability that a distinguisher $D_{0}$ returns true for some game $G_{0}$, we consider any context $C$ acceptable for $G_{0}$ with public variables $V$ and that does not contain events, and bound $\operatorname{Pr}\left[C\left[G_{0}\right]\right.$ : $D_{0} \wedge \neg$ NonUnique $]$ which is equal to $\operatorname{Pr}\left[C\left[G_{0}\right]: D_{0}\right]$ because no find [unique] occurs in the initial game. For each game transformation, we assume that the introduced variables are fresh, so that $C$ remains acceptable for all games of the sequence. We can then apply Lemma 1 for each game transformation. Points 1, 2, and 3 of this lemma allow us to handle several events simultaneously, as long as the proof uses the same sequence of games to bound their probabilities. Point 2 is useful for transformations that rely on the uniqueness of the values that satisfy the conditions of find, detailed in Appendix E-B: these transformations preserve the behavior of the game when $G$ does not execute event NonUnique. The distinguisher $D$ is always of the desired form $\left(D_{0} \wedge \neg\right.$ NonUnique $) \vee e_{1} \vee \ldots \vee e_{n}$ because we start from $D_{0} \wedge \neg$ NonUnique and add events introduced by Shoup's lemma using point 1 ; we abort immediately after these events. When the proof uses different sequences of games to bound the probabilities of events, we use point 4 of the lemma to bound each probability separately and compute the sum. The standard computation of probabilities corresponds to always applying point 4.

For example, suppose that we want to bound the probability of event $e_{0}$ in $G_{0}, G_{1}$ differs from $G_{0}$ only when $G_{1}$ executes event $e, G_{1} \approx_{p} G_{2}$, and $G_{2}$ executes neither $e_{0}$ nor $e$. Suppose for simplicity that no find [unique] occurs, so that NonUnique never occurs. Lemma 1 yields $\operatorname{Pr}\left[C\left[G_{0}\right]: e_{0}\right] \leq$ $\operatorname{Pr}\left[C\left[G_{1}\right]: e_{0} \vee e\right] \leq p\left(C, t_{e_{0} \vee e}\right)+\operatorname{Pr}\left[C\left[G_{2}\right]: e_{0} \vee e\right]=$ $p\left(C, t_{e_{0} \vee e}\right)$. The standard computation of probabilities yields $\operatorname{Pr}\left[C\left[G_{0}\right]: e_{0}\right] \leq \operatorname{Pr}\left[C\left[G_{1}\right]: e_{0}\right]+\operatorname{Pr}\left[C\left[G_{1}\right]: e\right] \leq$ $p\left(C, t_{e_{0}}\right)+p\left(C, t_{e}\right)$. The runtime $t_{D}$ of $D$ is approximately the same for $e_{0}, e$, and $e_{0} \vee e$, so $\operatorname{Pr}\left[C\left[G_{0}\right]: e_{0}\right] \leq p\left(C, t_{D}\right)$ by Lemma 1 , while $\operatorname{Pr}\left[C\left[G_{0}\right]: e_{0}\right] \leq 2 p\left(C, t_{D}\right)$ by the standard computation, so we have gained a factor 2 .

For secrecy, the advantage $\operatorname{Pr}\left[C\left[G \mid R_{x}\right]: \mathrm{S}\right]-\operatorname{Pr}[C[G$ $\left.\left.R_{x}\right]: \overline{\mathrm{S}}\right]$ introduces a factor 2 in the probability: if $G \approx_{p}^{\{x\}}$ $G^{\prime}$, then $\operatorname{Pr}\left[C\left[G \mid R_{x}\right]: \mathrm{S}\right]-\operatorname{Pr}\left[C\left[G \mid R_{x}\right]: \overline{\mathrm{S}}\right] \leq \mathbf{2} p(C[[] \mid$ $\left.\left.R_{x}\right], t_{\mathrm{S}}\right)+\left(\operatorname{Pr}\left[C\left[G^{\prime} \mid R_{x}\right]: \mathrm{S}\right]-\operatorname{Pr}\left[C\left[G^{\prime} \mid R_{x}\right]: \overline{\mathrm{S}}\right]\right)$, since $t_{\mathrm{S}}=t_{\overline{\mathrm{S}}}$. The next lemma avoids this factor 2 for probabilities of events:

Lemma 2 Let $C$ be a context acceptable for $G$ and $G^{\prime}$ 
with public variables $V$. Let the distinguishers $D, D^{\prime}$ be disjunctions of events $e_{1} \vee \ldots \vee e_{n}$ such that we abort just after executing each $e_{i}$. Let $\operatorname{Adv}_{G}^{\text {Secrecy }}(C, D)=\operatorname{Pr}[C[G \mid$ $\left.\left.R_{x}\right]: \mathrm{S} \vee D\right]-\operatorname{Pr}\left[C\left[G \mid R_{x}\right]: \overline{\mathrm{S}} \vee\right.$ NonUnique $]$.

1) If $G^{\prime}$ differs from $G$ only when $G^{\prime}$ executes event $e$ and we abort just after executing $e$, then $\operatorname{Adv}_{G}^{\text {Secrecy }}(C, D) \leq \operatorname{Adv}_{G^{\prime}}^{\text {Secrecy }}(C, D \vee e)$.

2) If $G$ differs from $G^{\prime}$ only when $G$ executes NonUnique, then $\operatorname{Adv}_{G}^{\text {Secrecy }}(C, D) \leq \operatorname{Adv}_{G^{\prime}}^{\text {Secrecy }}(C, D)$.

3) If $G \underset{p}{V} G^{\prime}$, then $\operatorname{Adv}_{G}^{\text {Secrecy }}(C, D) \leq 2 p(C[[]$ $\left.\left.R_{x}\right], t\right)+\operatorname{Adv}_{G^{\prime}}^{\text {Secrecy }}(C, D)$ where $t=\max \left(t_{\mathrm{S} \vee D}\right.$, $\left.t_{\overline{\mathrm{S}} \vee \text { NonUnique }}\right)$.

4) $\operatorname{Adv}_{G}^{\text {Secrecy }}\left(C, D \vee D^{\prime}\right) \leq \operatorname{Adv}_{G}^{\text {Secrecy }}(C, D)+\operatorname{Pr}[C[G \mid$ $\left.\left.R_{x}\right]: D^{\prime}\right]$.

5) If CryptoVerif proves the secrecy of $x$ in game $G$, then $\operatorname{Pr}\left[C\left[G \mid R_{x}\right]: \mathrm{S}\right]=\operatorname{Pr}\left[C\left[G \mid R_{x}\right]: \overline{\mathrm{S}}\right]$, so $\operatorname{Adv}_{G}^{\text {Secrecy }}(C, D) \leq \operatorname{Pr}\left[C\left[G \mid R_{x}\right]: D\right]$.

In order to prove secrecy of $x$ in the initial game $G_{0}$, we bound $\operatorname{Pr}\left[C\left[G_{0} \mid R_{x}\right]: \mathrm{S}\right]-\operatorname{Pr}\left[C\left[G_{0} \mid R_{x}\right]\right.$ : $\overline{\mathrm{S}}]=\operatorname{Adv}_{G_{0}}^{\text {Secrecy }}(C$, false $)$, by applying Lemma 2 for each game transformation. When we apply points 4 and 5 of this lemma, we use bounds on the probabilities of events, $\operatorname{Pr}\left[C\left[G \mid R_{x}\right]: D^{\prime}\right]$ and $\operatorname{Pr}\left[C\left[G \mid R_{x}\right]: D\right]$ respectively, which can be established using Lemma 1. (They can be written $\operatorname{Pr}\left[C\left[G \mid R_{x}\right]\right.$ : (false $\wedge \neg$ NonUnique $\left.) \vee D\right]$, so they are of the form required by point 2 of Lemma 1.) These probabilities are not multiplied by 2 , so we improve over the standard computation of probabilities for secrecy.

These improvements are implemented in CryptoVerif but also apply to manual proofs. For instance, by applying this result to the manual proof of OEKE [55], we obtain that the probability for any adversary to make a server instance accept with no terminating client partner is bounded by

$$
\begin{aligned}
& \frac{N_{U}+N_{S}}{N}+q_{h} \operatorname{Succ}_{\mathbb{G}}^{\text {cdh }}\left(t^{\prime}\right)+p_{\text {coll }}^{\prime} \\
& \text { with } p_{\text {coll }}^{\prime}=\frac{\left(2 q_{e}+2 N_{U}+3 N_{S}+3 N_{P}\right)^{2}}{2(q-1)}+\frac{q_{h}^{2}+2 N_{S}}{2^{l_{1}+1}}
\end{aligned}
$$

and that no adversary can distinguish the session key from a random key with advantage greater than

$$
\frac{N_{U}+N_{S}}{N}+q_{h} \operatorname{Succ}_{\mathbb{G}}^{\text {cdh }}\left(t^{\prime}\right)+2 p_{\text {coll }}^{\prime}
$$

with the notations of Section II. (The detailed computation is in Appendix D.) For both properties, the first term of the probability $\frac{N_{U}+N_{S}}{N}$ shows that the adversary can test at most one password for each interaction with the client or the server, which is the optimal result, while the standard evaluation of probabilities given in Section II yields $\frac{N_{U}+\mathbf{2} N_{S}}{N}$ for the first property and $\frac{\mathbf{2} N_{U}+\mathbf{4} N_{S}}{N}$ for the second one. Similar improvements could also be obtained for the AuthA protocol [55, Section 4.1] and for the forward secrecy property [55, Appendix D].
1) Inserting events: In order to introduce events, we have implemented a new game transformation in CryptoVerif: insert_event $e o$ inserts event_abort $e$ at program point $o$. The construct event_abort $e$ executes event $e$ and aborts the game. The program point $o$ is an integer, which can be determined using the command show_game occ: this command displays the current game with the corresponding label $\{o\}$ at each program point. The command show_game occ also allows one to inspect the game, for instance to know the names of fresh variables created by CryptoVerif during previous transformations. Program points and variable names may depend on the version of CryptoVerif; this paper uses CryptoVerif 2.06. Since CryptoVerif version 2.01, program points can also be designated by expressions like before regexp, which designates the program point at the beginning of the line that matches the regular expression regexp, or after regexp, which designates the program point just after the line that matches regexp. This way of designating program points is more stable across versions of CryptoVerif. CryptoVerif cannot guess where events should be introduced, so the command insert_event must be manually given to the tool.

We have also defined a command insert $o$ ins which adds instruction ins at the program point $o$. The instruction ins can for instance be a test, in which case all branches of the test will be copies of the code that follows program point $o$ (so that the semantics of the game is unchanged). It can also be an assignment or a random generation of a fresh variable or an event_abort instruction. In all cases, CryptoVerif checks that this instruction preserves the semantics of the game except when we execute an inserted event_abort, and rejects it with an error message if it does not.

2) Transformations for random oracles: At the beginning of the proof, we transform the game using the random oracle assumption for h0. That will help us insert appropriate events. Before actually performing this transformation, we first introduce a case distinction that leads to a simpler game after applying the random oracle assumption:

- By command insert after "OH(" "let concat $(x 01$, $x 02, x 03, x 04, x 05)=x 1$ in”, we introduce a let in the hash oracle $\mathrm{OH}$ for h0. As the result, this hash oracle becomes $\mathrm{OH}(x 1$ : bitstring $):=$ let concat $(x 01$, $x 02, x 03, x 04, x 05)=x 1$ in return $(\mathrm{h} 0(h k 0, x 1))$ else $\operatorname{return}(\mathrm{hO}(h k 0, x 1))$ : the meaning of this let construct is that, if $x 1$ is of the form concat $(x 01, x 02, x 03$, $x 04, x 05)$, then the in branch is taken with $x 01, x 02$, $x 03, x 04, x 05$ bound to their value (which is uniquely determined because the length of the fields of the concatenation is fixed); otherwise, the else branch is taken. Thus, we distinguish cases depending on whether $x 1$ is of the form concat $(\ldots)$ or not. In the next transformation, which applies the random oracle assumption to h0, we are going to replace calls to h0 with lookups in the previous queries to h0. All queries 
to h0 in the protocol have an argument of the form concat $\left(x 01^{\prime}, x 02^{\prime}, x 03^{\prime}, x 04^{\prime}, x 05^{\prime}\right)$. When comparing this query to a query in the hash oracle, the comparison $x 1=\operatorname{concat}\left(x 01^{\prime}, x 02^{\prime}, x 03^{\prime}, x 04^{\prime}, x 05^{\prime}\right)$ can then be simplified as follows:

- If h0(x1) was computed in the in branch of the introduced let, the comparison becomes concat $(x 01$, $x 02, x 03, x 04, x 05)=\operatorname{concat}\left(x 01^{\prime}, x 02^{\prime}, x 03^{\prime}\right.$, $\left.x 04^{\prime}, x 05^{\prime}\right)$, that is, $x 01=x 01^{\prime} \wedge \ldots \wedge x 05=x 05^{\prime}$.

- If $\mathrm{h} 0(x 1)$ was computed in the else branch of this let, the comparison becomes false, because $x 1$ cannot be of the form concat $(. .$.$) , since the$ in branch would have been taken in that case.

- crypto rom(h0) applies the equivalence $L_{1} \approx_{p_{1}} R_{1}$ of Figure 2, designated by rom for Random Oracle Model, to the hash function h0: it transforms calls to h0 into lookups in the previous queries to h0, as outlined in Section III-B.

Next, we proceed similarly for $\mathrm{h} 1$ :

- insert after "OH_1" "let concat $(x 11, x 12, x 13$, $x 14, x 15)=x 1_{-} 1$ in

- crypto rom(h1)

3) Event Auth: Next, we introduce event Auth: This event corresponds to the case in which the group element $X$ received by the server (denoted $X_{-} s$ ) does not come from the client, the authenticator Auth received by the server (denoted auth_s) comes from a hash query by the adversary, and authentication still succeeds. This event is introduced as follows:

- insert after "OS2" "find $j \leq N U$ suchthat defined $(X[j]) \wedge X[j]=X \_s$ then else find $j h \leq$ $q H 1$ suchthat $\operatorname{defined}(x 11[j h], \quad x 12[j h], \quad x 13[j h]$, $\left.x 14[j h], r_{-} 6[j h]\right) \wedge(\mathrm{U}=x 11[j h]) \wedge(\mathrm{S}=x 12[j h]) \wedge$ $\left(X \_s=x 13[j h]\right) \wedge(Y=x 14[j h]) \wedge($ auth_s $=$ $\left.r \_b[j h]\right)$ then event_abort Auth" first inserts a test after receiving the authenticator in the server, to distinguish the case in which $X_{-} s$ comes from the client $\left(X_{-} s=X[j]\right.$ for some $\left.j\right)$. In the else branch of this test, it inserts a test to detect when authentication succeeds with an authenticator auth_s that comes from a hash query made by the adversary. The result of that hash query is $r_{-} 6[j h]$ and its arguments are $x 11[j h]$, $\ldots, x 15[j h]$. We purposely do not test that the 5th argument of the hash query is the expected one. This avoids computing an exponentiation $\exp \left(X \_s, y\right)$ where $X_{\_} s$ comes from the adversary and $y$ is a secret exponent, thus removing a query to $\mathrm{Ob}$ in the $\mathrm{CDH}$ equivalence. The event Auth itself is inserted in the then branch of the previous test.

- simplify cleans up the obtained game. The else branch of the find $j h$ inserted above is removed: in that branch, authentication always fails so the protocol executes nothing.
4) Event Encrypt: Next, we introduce the event Encrypt: This event corresponds to the case in which the value $Y^{\star}$ received by the client (denoted Ystar_u) comes from an encryption query of the adversary under the correct password. As above, we have to prepare this insertion:

- crypto $\mathrm{icm}(\mathrm{enc})$ applies the equivalence that represents the Ideal Cipher Model, designated by icm, to the encryption scheme enc: it replaces calls to encryption/decryption with lookups in previous queries, as outlined in Section III-C.

- insert_event Encrypt after "pw0 =ke \\" inserts the event Encrypt when the lookup in previous encryption/decryption queries that comes from the decryption of Ystar_u succeeds with an encryption query of the adversary.

5) Event cdh: Next, we introduce the event cdh. This event corresponds to cases in which the adversary would break the computational Diffie-Hellman assumption.

- crypto group_to_exp_strict(exp) * applies equivalence (8) as many times as possible. It applies it twice: it replaces the generation of a fresh group element $X$ with the generation of an exponent $x$ and the computation $X \leftarrow \exp (\mathrm{g}, x)$ as a result of decryption when no previous encryption/decryption query matches, in the decryption oracle and in the client.

- insert before "find $\backslash \backslash[$ unique $\backslash \backslash]$ u_23"

"find $i 7 \leq N U, i 8 \leq N U$ suchthat defined $(x[i \eta]$, $\left.X[i 7], x \_3[i 8]\right) \wedge\left(x 14=\exp \left(\mathrm{g}, x_{-} 3[i 8]\right)\right) \wedge(x 13=$ $X[i 7]) \wedge(x 12=\mathrm{S}) \wedge(x 11=\mathrm{U}) \wedge(x 15=$ $\left.\exp \left(\mathrm{g}, \operatorname{mult}\left(x_{-} 3[i 8], x[i \gamma]\right)\right)\right)$ then

event_abort $\mathrm{cdh}$

orfind $i \eta \leq N U, i 9 \leq q D$ suchthat defined $(x[i 7]$, $\left.X[i 7], x_{2} 2[i 9]\right) \wedge\left(x 14=\exp \left(\mathrm{g}, x_{2} 2[i 9]\right)\right) \wedge(x 13=$ $X[i 7]) \wedge(x 12=\mathrm{S}) \wedge(x 11=\mathrm{U}) \wedge(x 15=$ $\left.\exp \left(g, \operatorname{mult}\left(x \_2[i 9], x[i \eta]\right)\right)\right)$ then

event_abort cdh

orfind $i \eta \leq N U, i 10 \leq N S$ suchthat defined $(x[i 7]$, $y[i 10], X[i 7], Y[i 10]) \wedge(x 14=Y[i 10]) \wedge(x 13=$ $X[i 7]) \wedge(x 12=\mathrm{S}) \wedge(x 11=\mathrm{U}) \wedge(x 15=$ $\exp (\mathrm{g}, \operatorname{mult}(y[i 10], x[i \eta])))$ then

event_abort cdh

orfind $i \eta \leq N U, i 11 \leq N P$ suchthat defined $(x[i 7]$, $y p[i 11], X[i 7], Y p[i 11]) \wedge(x 14=Y p[i 11]) \wedge(x 13=$ $X[i 7]) \wedge(x 12=\mathrm{S}) \wedge(x 11=\mathrm{U}) \wedge(x 15=\exp (\mathrm{g}$, mult $\left.\left.\left(y p[i 11], x\left[i^{r}\right]\right)\right)\right)$ then

event_abort cdh

orfind $i 6 \leq N P$ suchthat defined $(y p[i 6], x p[i 6]$, $X p[i 6], Y p[i 6]) \wedge(x 14=Y p[i 6]) \wedge(x 13=X p[i 6]) \wedge$ $(x 12=\mathrm{S}) \wedge(x 11=\mathrm{U}) \wedge(x 15=\exp (\mathrm{g}, \operatorname{mult}(x p[i 6]$, $y p[i 6])))$ then

event_abort cdh" rewrites the first find in the oracle $O H_{-} 1$ for h1, to capture exactly the conditions that can be eliminated by $\mathrm{CDH}$. The conditions are all of the 
form: $i, j$ suchthat defined $(x[i], y[j]) \wedge(x 14=\exp (\mathrm{g}$, $x[i])) \wedge(x 13=\exp (\mathrm{g}, y[j])) \wedge(x 12=\mathrm{S}) \wedge(x 11=$ $\mathrm{U}) \wedge(x 15=\exp (\mathrm{g}, \operatorname{mult}(x[i], y[j])))$ for some exponents $x, y$. (We sometimes replace $\exp (\mathrm{g}, x[i])$ with a variable access when its value was already computed before, and similarly for $y[j]$.) These conditions are true as soon as the exponents have been computed and the arguments of the hash query are well chosen. That allows the subsequent simplification to eliminate similar conditions in the rest of the game (because if those conditions were true, the game would have already executed event cdh and aborted). Furthermore, we test $x 15=\exp (\mathrm{g}, \operatorname{mult}(x[i], y[j]))$ as last condition, so that the test is executed only when $x 13$ and $x 14$ are the right public keys. CryptoVerif then realizes that the test is made for a single $i, j$ for each hash query. (The values of $i$ and $j$ are fixed when $x 13$ and $x 14$ are given.) That allows later CryptoVerif to bound the probability of event cdh by $q H 1 \cdot \operatorname{Succ}_{\mathbb{G}}^{\text {cdh }}\left(t^{\prime}\right)$ for some runtime $t^{\prime}$.

- insert before "find $\backslash \backslash\left[\right.$ unique $\backslash \backslash u_{-} 7$ " "find $i 19 \leq N U, i 20 \leq N U$ suchthat defined $\left(X[i 19], x[i 19], x \_3[i 20]\right) \wedge(x 04=\exp (\mathrm{g}$, $\left.\left.x_{-} 3[i 20]\right)\right) \wedge(x 03=X[i 19]) \wedge(x 02=\mathrm{S}) \wedge(x 01=$ $\mathrm{U}) \wedge\left(x 05=\exp \left(\mathrm{g}, \operatorname{mult}\left(x \_3[i 20], x[i 19]\right)\right)\right)$ then event_abort cdh

orfind $i 17 \leq N U, i 21 \leq q D$ suchthat $\operatorname{defined}\left(X[i 17], x[i 17], x \_2[i 21]\right) \wedge(x 04=\exp (\mathrm{g}$, $\left.\left.x \_2[i 21]\right)\right) \wedge(x 03=X[i 17]) \wedge(x 02=\mathrm{S}) \wedge(x 01=$ $\mathrm{U}) \wedge\left(x 05=\exp \left(\mathrm{g}, \operatorname{mult}\left(x_{2} 2[i 21], x[i 17]\right)\right)\right)$ then event_abort cdh

orfind $i 15 \leq N U, i_{22} \leq N S$ suchthat defined $(X[i 15], Y[i 22], x[i 15], y[i 22]) \wedge(x 04=$ $Y[i 22]) \wedge(x 03=X[i 15]) \wedge(x 02=\mathrm{S}) \wedge(x 01=$ $\mathrm{U}) \wedge(x 05=\exp (\mathrm{g}, \operatorname{mult}(y[i 22], x[i 15])))$ then event_abort cdh

orfind $i 13 \leq N U$, i23 $\leq N P$ suchthat defined $(X[i 13], Y p[i 23], x[i 13], y p[i 23]) \wedge(x 04=$ $Y p[i 23]) \wedge(x 03=X[i 13]) \wedge(x 02=\mathrm{S}) \wedge(x 01=$ $\mathrm{U}) \wedge(x 05=\exp (\mathrm{g}, \operatorname{mult}(y p[i 23], x[i 13])))$ then

event_abort cdh" performs a similar transformation for the oracle for $h 0$.

- simplify then removes all comparisons of the form $x 15=\exp (\mathrm{g}, \operatorname{mult}(x, y))$ and $x 05=\exp (\mathrm{g}, \operatorname{mult}(x$, $y)$ ) except those in the two inserted instructions above: they are all false, because if they were true, the game would have executed event cdh and aborted before.

\section{Proving the security properties}

- success tries to prove all security properties. At this point, CryptoVerif can bound the probability of breaking all desired properties (secrecy of $s k \_u$, correspondence (9)). However, the obtained bound depends on the probability of executing the events Auth, Encrypt, and cdh which are not eliminated yet, so the proof continues in order to eliminate these events.

E. Bounding the probability of event $\mathrm{cdh}$ and cleaning up

- crypto cdh(exp) applies the CDH assumption (Section III-D). The oracles $\mathrm{Oa}$ and $\mathrm{Ob}$ are in fact not used in this example (the code that would use them has been removed by introducing events), so the situation is particularly simple: expressions of the form $m=\exp (\mathrm{g}, \operatorname{mult}(a[i], b[j]))$ are replaced with false. That removes the cdh event introduced above.

- After applying the CDH assumption, the exponents $x$ are only used in $\exp ^{\prime}(\mathrm{g}, x)$. By crypto $\exp ^{\prime}$ _to_group $(\exp ) *$, we apply the version of equivalence (6) for exp'. It replaces the computation of $\exp ^{\prime}(\mathrm{g}, x)$ for a fresh random $x$ with the generation of a random group element $X$, for the result of the decryption oracle and for the computations of $Y p$ and $X p$ (the values of $Y$ and $X$ in passive eavesdroppings), $Y$ (in the server), $Y_{-} u$ and $X$ (in the client).

- SArename $Y_{-} u$ renames each occurrence of $Y_{-} u$ to a distinct name. Indeed, as part of crypto icm(enc), the definition of $Y_{-} u$ has been copied once for each possible origin (a previous encryption/decryption query, or a fresh $Y_{-} u$ if no previous query matches). After this renaming, CryptoVerif replaces each usage of $Y_{-} u$ with its value and simplifies comparisons that involve $Y \_u$.

\section{F. Reorganizing Random Number Generations}

We end up in a situation in which random values for $Y$ are generated, but are used only in comparisons with previous queries. We would like to delay or remove these random number generations. This situation occurs at three places:

- When $Y \_u$ (the value of $Y$ in the client) is a fresh random group element, auth_u and $K \_u$ are also fresh random values, independently of the value of $Y_{-} u$, so $Y_{-} u$ is used only in comparisons with previous encryption/decryption queries.

- The value of $Y$ in the passive eavesdroppings, $Y p$, is a fresh random group element; the encryption $Y^{\star}$ of $Y$ is thus also a fresh random group element by the ideal cipher model, and the hash queries return a random value independently of the value of $Y p$, so $Y p$ is also used only in comparisons with previous encryption/decryption queries.

- The value of $Y$ in the server is also a fresh random group element; it is used in the test that decides whether to execute event Auth and in comparisons with previous encryption/decryption queries.

We have implemented new game transformations in CryptoVerif, detailed in Appendix E, to handle this situation:

- move array $X$ delays the generation of a random value $X$ until the point at which it is first used. 
- merge_arrays $x_{11} \ldots x_{1 n}, \ldots, x_{m 1} \ldots x_{m n}$ merges the variables $x_{j 1}, \ldots, x_{j n}$ into a single variable $x_{j 1}$ for each $j \leq m$. Each variable $x_{j k}$ must have a single definition. For each $j \leq n$, the variables $x_{j 1}, \ldots$, $x_{j n}$ must have the same type and indices of the same type. They must not be defined for the same value of their indices (that is, $x_{j k}$ and $x_{j k^{\prime}}$ must be defined in different branches of if or find when $k \neq k^{\prime}$ ), so that they can be merged into a single array.

- merge_branches merges branches of if and find when they execute the same code.

Using these transformations, we can eliminate the random number generations for $Y$ as outlined at the beginning of this section. We consider the three generations of $Y$ in turn. For each of these generations, we first apply move array to the corresponding variable, to delay its generation. For OEKE, this has the effect of generating it in the decryption oracle available to the adversary. So, in this oracle, we end up with two possibilities of generating a fresh result, the one that comes from the delayed generation of $Y$, say $Y^{\prime}$, and the one that corresponds to the situation in which the query is really a fresh decryption query, say $Y_{d}$. As part of the simplification that follows move array, CryptoVerif tries to merge these two cases by merge_branches. However, merge_branches does not succeed directly: we first need to merge the two variables $Y_{d}$ and $Y^{\prime}$ into a single variable by merge_arrays $Y_{d} Y^{\prime}$, then we can apply merge_branches. The transformation merge_branches automatically advises CryptoVerif to perform merge_arrays $Y_{d} Y^{\prime}$ and then retry merge_branches.

In more detail, we proceed as follows, considering the three generations of $Y$ in turn.

- First, $Y_{u}$ (the value of $Y$ in the client), when it is a fresh random group element. This variable is now named $X_{-} 5$, so we use the command move array $X_{-} 5$ to delay its generation. The game is automatically simplified after this command, which reorganizes find constructs. Before move array, $X_{-} 5$ is used in the following ways:

1) to compare it with the argument of encryption queries in the encryption oracle. If $X_{-} 5$ is a fresh random value at this point, the comparison is true with probability $1 /|G|$. The transformation move array uses that property to avoid generating a random value for $X_{-} 5$ at this point and to delay the generation further.

2) as a result of decryption queries in the decryption oracle. The generation of $X_{-} 5$ in the client is replaced with a generation of $Y_{-} 1$ in the decryption oracle.

3) as a result of the decryption query $\operatorname{dec}\left(Y^{\star}, p w\right)$ in the client. That result is unused, so $X_{-} 5$ appears only in a defined condition, which CryptoVerif leaves unchanged.

Therefore, in the decryption oracle, when the result was not generated before, we have two cases: either the result was in fact an $Y_{u}$ whose generation has been delayed, and it is now generated as $Y_{-} 1$, or the query is really a fresh decryption query, and the result is named $X_{-} 1$. Unfortunately, the advised merge_arrays $X_{-} 1 Y_{-} 1$ is weaker than the manual one: it does not allow creating branch variables. (The details of merge_arrays and branch variables are explained in Appendix E-C.) This advised version fails, so we have to manually perform merge_arrays $X_{-}{ }_{1} Y_{-} 1$ to merge $Y_{-} 1$ into $X_{-} 1$ and finally merge_branches.

- Second, the value of $Y$ in passive eavesdroppings, $Y p$. This variable is now named $X_{-} 2$. We proceed similarly, using move array $X_{-} 2$. Then CryptoVerif automatically merges branches, by first merging the delayed $X_{-} 2$ named $Y_{-} 3$ into the result of decryption $X_{-} 1$ by applying merge_arrays $X_{-} 1 Y_{-} 3$, and finally applying merge_branches.

- Third, the value of $Y$ in the server, now named $X_{-} 4$. We again proceed similarly, using move array $X_{-} 4$. Then CryptoVerif automatically merges the delayed $X_{-} 4$ named $Y_{-} 4$ into the result of decryption $X_{-} 1$ by applying merge_arrays $X_{-} 1 Y_{-} 4$. It cannot apply merge_branches directly because the condition that triggers the event Auth,

$$
\begin{aligned}
& \text { find } j h=j h \_1 \leq q H 1, u \_78=r i \_98 \leq q D \\
& \text { suchthat defined }\left(x 11\left[j h \_1\right], x 12\left[j h \_1\right], x 13\left[j h \_1\right],\right. \\
& \left.x 14\left[j h \_1\right], r \_6\left[j h \_1\right], u \_80\left[r i \_98\right], X_{1}\left[r i \_98\right]\right) \wedge \\
& \left(u \_80\left[r i \_98\right]=i S\right) \wedge\left(\mathrm{U}=x 11\left[j h \_1\right]\right) \wedge \\
& \left(\mathrm{S}=x 12\left[j h \_1\right]\right) \wedge\left(X \_s=x 13\left[j h_{-} 1\right]\right) \wedge \\
& \left(x 14\left[j h \_1\right]=X_{-} 1\left[r i \_98\right]\right) \wedge\left(\text { auth_s }=r \_6\left[j h \_1\right]\right)
\end{aligned}
$$

then

refers to the variable $u \_80$ which is defined in the condition of a find whose branches we would like to merge; this merging is not possible because it would make the definition of $u_{-} 80$ disappear. So we manually rewrite the condition (11) to remove the reference to u_80. By

$$
\begin{aligned}
& \text { insert before "find } j h \text { " "find } j h^{\prime} \leq q H 1, j d \leq q D \\
& \text { suchthat defined }\left(x 11\left[j h^{\prime}\right], x 12\left[j h^{\prime}\right], x 13\left[j h^{\prime}\right],\right. \\
& \left.x 14\left[j h^{\prime}\right], r \_6\left[j h^{\prime}\right], m[j d], k d[j d], X_{-} 1[j d]\right) \wedge \\
& \left(m[j d]=m d \_O \_e n c\right) \wedge\left(\mathrm{U}=x 11\left[j h^{\prime}\right]\right) \wedge \\
& \left(\mathrm{S}=x 12\left[j h^{\prime}\right]\right) \wedge\left(X \_s=x 13\left[j h^{\prime}\right]\right) \wedge \\
& \left(x 14\left[j h^{\prime}\right]=X \_1[j d]\right) \wedge\left(a u t h \_s=r \_6\left[j h^{\prime}\right]\right) \wedge \\
& (k d[j d]=p w 0) \text { then event_abort Auth2" }
\end{aligned}
$$


we insert a test just above the find (11). The variables jh_1 and ri_98 of the find (11) are renamed to $j h^{\prime}$ and $j d$ respectively in (12) (without change in the meaning), and the condition $u_{-} 80[$ ri_98] $=i S$ of (11), that is, $u_{-} 80[j d]=i S$ is replaced with $m[j d]=m d \_O_{-} e n c \wedge k d[j d]=p w 0$ in (12). In the considered game, the variable $u \_80$ is defined in a find with condition $m=m d_{-} O_{-} e n c\left[u_{-} 80\right] \wedge$ $k d=p w 0$, so when $u_{-} 80[j d]$ is defined, we have $m[j d]=m d \_O \_e n c\left[u \_80[j d]\right] \wedge k d[j d]=p w 0$; when $u \_80[j d]=i S$, we obtain exactly the condition of (12), knowing that the index $i S$ can be omitted because it is the index of the replication above the find (12). Explained another way, the find of (12) looks for a hash query indexed by $j h^{\prime}$ and a decryption query indexed by $j d$, such that the adversary has decrypted the value of $Y^{\star}$ generated by the server, $m d_{-} O_{-} e n c$, under the correct password $p w 0$, obtaining $X_{-} 1[j d]$ (which is then the correct value of $Y$ ) using that decryption query, and then has passed a value (U, $\left.\mathrm{S}, X_{-} s, X_{-} 1[j d],{ }_{-}\right)$ to the hash query, obtaining the correct authenticator auth_s $=r_{-} 6\left[j h^{\prime}\right]$. This corresponds exactly to the situation in which the adversary authenticates to the server by guessing the password. CryptoVerif can show that the condition (11) implies the condition of (12), so in the else branch of (12), the condition (11) never holds, hence by simplification (simplify), we remove that else branch. Then merge_branches succeeds. At this point, event Auth does not occur in the current game. (It has been replaced by Auth2 in (12).)

\section{G. The Final Computation of Probabilities}

In the obtained game, the events Auth2 and Encrypt are guarded by the following conditions (some variables have been renamed for readability):

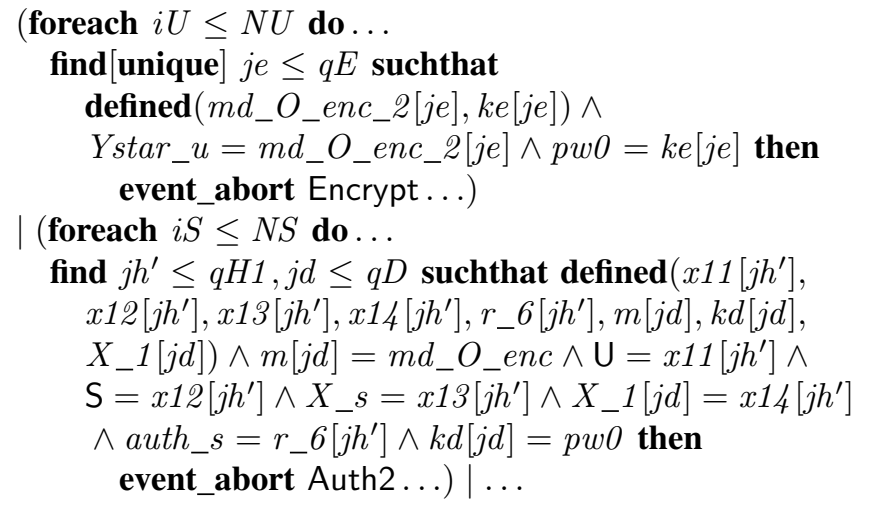

So, in order to bound the probabilities of these events, we just have to eliminate collisions between the password $p w 0$ and the encryption and decryption keys, $k e[j e]$ and $k d[j d]$. To do this, we first allow eliminating collisions whose probability is bounded by $n /|T|$ where $n$ is a number of calls of estimate at most small and $T$ is a type of size estimate at least password, in addition to collisions that can already be eliminated, by the command allowed_collisions noninteractive 2 /large, small/password. The elimination of collisions itself is done by the command simplify coll_elim(variables : $p w 0)$. The collisions on $p w 0$ are not eliminated automatically by CryptoVerif because the type passwd of $p w 0$ is declared with annotation password. This annotation allows manual elimination of collisions but prevents automatic elimination of collisions. For passwords, whose set is not very large, the automatic elimination of collisions would yield a too large probability bound.

We have to evaluate the probability of these collisions. A naive evaluation considers that one makes at most $N U \times q E$ comparisons $p w 0=k e[j e]$ (there are $N U$ sessions of the client and the condition of find is evaluated at most $q E$ times) and similarly at most $N S \times q H 1 \times q D$ comparisons $k d[j d]=p w 0$, which yields the probability $(N U \times q E+$ $N S \times q H 1 \times q D) / \mid$ passwd $\mid$. A slightly more clever way is to notice that $p w 0=k e[j e]$ contains as only index $j e \leq q E$, so at most $q E$ distinct comparisons are performed (there are at most $q E$ distinct encryption keys), and similarly at most $q D$ distinct comparisons $k d[j d]=p w 0$, which yields the probability $(q E+q D) / \mid$ passwd $\mid$. This is not satisfactory yet, because the encryption and decryption queries can be performed by the adversary without interacting with the protocol, so $q E$ and $q D$ can be large. So we have extended CryptoVerif to improve this probability bound. We start from the most naive evaluation $N U \times q E$ and try to eliminate each factor. We can eliminate $N U$ as shown above, but we can also eliminate $q E$ : for each session of the client, $Y s t a r_{-} u$ is fixed; since Ystar_u=md_O_enc_2[je], $m d \_O \_e n c \_2[j e]$ is also fixed. By eliminating collisions on $m d \_O \_e n c \_2$, there is a unique $j e$ that can make the comparison Ystar_u $=m d_{-} O_{-} e n c_{-} 2[j e]$ succeed, so a unique $j e$ for which the comparison $p w 0=k e[j e]$ is performed. Similarly, the comparison $k d[j d]=p w 0$ is performed at most once for each session of the server. Thus we obtain the probability $(N U+N S) / \mid$ passwd $\mid$. Since $q E$ and $q D$ are annotated noninteractive, they are larger than $N U$ and $N S$, annotated small. Therefore, the bound $(N U+N S) / \mid$ passwd $\mid$ is better than $(q E+q D) / \mid$ passwd $\mid$, so CryptoVerif returns the former.

CryptoVerif then concludes that the events Encrypt and Auth2 can be executed with probability at most $(N U+$ $N S) / \mid$ passwd $\mid$ in the last game. Finally, CryptoVerif shows that OEKE preserves the secrecy of $s k_{-} u$ up to probability

$$
\frac{N U+N S}{\mid \text { passwd } \mid}+q_{h} \operatorname{Succ}_{\mathbb{G}}^{\text {cdh }}\left(t^{\prime}\right)+\frac{4 q_{T}^{2}}{|G|}+\frac{q H 1^{2}+2 N S}{\mid \text { hash } 1 \mid},
$$

satisfies the correspondence (9) with public variables $\left\{s k \_u\right\}$ up to probability

$$
\frac{N U+N S}{\mid \text { passwd } \mid}+q_{h} \operatorname{Succ}_{\mathbb{G}}^{\text {cdh }}\left(t^{\prime}\right)+\frac{2 q_{T}^{2}}{|G|}+\frac{q H 1^{2}+N S}{\mid \text { hash } 1 \mid},
$$


and satisfies the correspondence (10) with public variables $\left\{s k_{-} u\right\}$ up to probability $N U^{2} / 2|G|$, where $t^{\prime}=t+$ $(q D+2 N U+2 N P+N S+1) \tau_{\text {exp }}, q_{h}=q H 0+q H 1$, $q_{T} \leq q_{h}+q E+q D+3(N U+N S+N P)=O(t)$, and $t$ is the runtime of the adversary. The main term in these probabilities is $(N U+N S) / \mid$ passwd $\mid$ : the adversary can test at most one password per session with the client or the server (active attack), which is the best bound we can hope. In contrast, [55] yields a bound of at most 4 passwords per session. In Section IV-C, by applying our improvement of the computation of probabilities to the manual proof of [55], we obtained the same first and second terms as CryptoVerif, and slightly better third and fourth terms.

\section{CONCLUSION}

We have proved the security of OEKE using the tool CryptoVerif. This proof provides additional confidence that the protocol is correct. Moreover, we have improved the probability bound given in [55]: we have shown that the adversary can test at most one password per session with the client or with the server, which is the optimal result. OEKE is a non-trivial case study, which is interesting on its own. It was also an opportunity to implement many extensions to CryptoVerif, which will be useful for proving many other protocols. We have already used the model of $\mathrm{CDH}$ to prove a signed Diffie-Hellman protocol. We plan to apply these extensions to other protocols, such as IKEv2 or $\mathrm{SSH}$, which also rely on Diffie-Hellman. Our improvement of the computation of probabilities is also of general interest, and applies to manual proofs as well as CryptoVerif proofs.

Acknowledgments: We thank David Pointcheval for his advice and help during this project. This work was partly supported by the ANR project ProSe (decision number ANR-2010-VERS-004-01).

\section{REFERENCES}

[1] B. Chor and R. L. Rivest, "A Knapsack type public key cryptosystem based on arithmetic in finite fields," in CRYPTO'84, ser. LNCS, vol. 196. Springer, 1985, pp. 54-65.

[2] H. W. Lenstra Jr., "On the Chor-Rivest knapsack cryptosystem," Journal of Cryptology, vol. 3, no. 3, pp. 149-155, 1991.

[3] S. Vaudenay, "Cryptanalysis of the Chor-Rivest cryptosystem," in CRYPTO'98, ser. LNCS, vol. 1462. Springer, 1998, pp. 243-256.

[4] M. Bellare and P. Rogaway, "The exact security of digital signatures: How to sign with RSA and Rabin," in EUROCRYPT'96, ser. LNCS, vol. 1070. Springer, 1996, pp. 399416.

[5] K. Ohta and T. Okamoto, "On concrete security treatment of signatures derived from identification,' in CRYPTO'98, ser. LNCS, vol. 1462. Springer, 1998, pp. 354-369.

[6] M. Bellare and P. Rogaway, "Optimal asymmetric encryption," in EUROCRYPT'94, ser. LNCS, vol. 950. Springer, 1994, pp. 92-111.
[7] V. Shoup, "OAEP reconsidered," Journal of Cryptology, vol. 15, no. 4, pp. 223-249, 2002.

[8] E. Fujisaki, T. Okamoto, D. Pointcheval, and J. Stern, "RSAOAEP is secure under the RSA assumption," Journal of Cryptology, vol. 17, no. 2, pp. 81-104, 2004.

[9] G. Barthe, B. Grégoire, S. Z. Béguelin, and Y. Lakhnech, "Beyond provable security. Verifiable IND-CCA security of OAEP," in CT-RSA 2011, ser. LNCS, vol. 6558. Springer, 2011, pp. 180-196.

[10] S. Halevi, "A plausible approach to computer-aided cryptographic proofs," Cryptology ePrint Archive, Report 2005/181, 2005, http://eprint.iacr.org/.

[11] M. Abadi and P. Rogaway, "Reconciling two views of cryptography (the computational soundness of formal encryption)," Journal of Cryptology, vol. 15, no. 2, pp. 103-127, 2002.

[12] V. Cortier and B. Warinschi, "Computationally sound, automated proofs for security protocols," in ESOP'05, ser. LNCS, vol. 3444. Springer, 2005, pp. 157-171.

[13] R. Janvier, Y. Lakhnech, and L. Mazaré, "Completing the picture: Soundness of formal encryption in the presence of active adversaries," in ESOP'05, ser. LNCS, vol. 3444. Springer, 2005, pp. 172-185.

[14] H. Comon-Lundh and V. Cortier, "Computational soundness of observational equivalence," in CCS'08. ACM, 2008, pp. 109-118.

[15] M. Backes, D. Hofheinz, and D. Unruh, "CoSP: A general framework for computational soundness proofs," in CCS'09. ACM, 2009, pp. 66-78.

[16] V. Cortier and B. Warinschi, "A composable computational soundness notion," in CCS'11. ACM, 2011, pp. 63-74.

[17] V. Cortier, S. Kremer, and B. Warinschi, "A survey of symbolic methods in computational analysis of cryptographic systems," Journal of Automated Reasoning, vol. 46, no. 3-4, pp. 225-259, 2011.

[18] V. Cortier, H. Hördegen, and B. Warinschi, "Explicit randomness is not necessary when modeling probabilistic encryption," in ICS 2006, ser. ENTCS, vol. 186. Elsevier, 2006, pp. 49-65.

[19] M. Backes, B. Pfitzmann, and M. Waidner, "A composable cryptographic library with nested operations," in CCS'03. ACM, 2003, pp. 220-230.

[20] M. Backes and B. Pfitzmann, "Symmetric encryption in a simulatable Dolev-Yao style cryptographic library," in CSFW'04. IEEE, 2004, pp. 204-218.

[21] C. Sprenger, M. Backes, D. Basin, B. Pfitzmann, and M. Waidner, "Cryptographically sound theorem proving," in CSFW'06. IEEE, 2006, pp. 153-166.

[22] C. Sprenger and D. Basin, "Cryptographically-sound protocol-model abstractions," in LICS'08. IEEE, 2008, pp. 3-17. 
[23] R. Canetti, "Universally composable security: A new paradigm for cryptographic protocols," in FOCS'01. IEEE, 2001, pp. 136-145.

[24] R. Canetti and J. Herzog, "Universally composable symbolic analysis of cryptographic protocols (the case of encryptionbased mutual authentication and key exchange)," Cryptology ePrint Archive, Report 2004/334, 2004, available at http:// eprint.iacr.org/2004/334.

[25] B. Blanchet, "Automatic proof of strong secrecy for security protocols," in IEEE Symposium on Security and Privacy, 2004, pp. 86-100.

[26] J. C. Mitchell, A. Ramanathan, A. Scedrov, and V. Teague, "A probabilistic polynomial-time calculus for the analysis of cryptographic protocols," Theoretical Computer Science, vol. 353, no. 1-3, pp. 118-164, 2006.

[27] D. Nowak and Y. Zhang, "A calculus for game-based security proofs," in ProvSec 2010, ser. LNCS, vol. 6402. Springer, 2010, pp. 35-52.

[28] A. Datta, A. Derek, J. C. Mitchell, V. Shmatikov, and M. Turuani, "Probabilistic polynomial-time semantics for a protocol security logic," in ICALP'05, ser. LNCS, vol. 3580. Springer, 2005, pp. 16-29.

[29] A. Datta, A. Derek, J. C. Mitchell, and B. Warinschi, "Computationally sound compositional logic for key exchange protocols," in CSFW'06. IEEE, 2006, pp. 321-334.

[30] G. Barthe, M. Daubignard, B. Kapron, and Y. Lakhnech, "Computational indistinguishability logic," in CCS'10. ACM Press, 2010, pp. 375-386.

[31] R. Canetti, L. Cheung, D. Kaynar, M. Liskov, N. Linch, O. Pereira, and R. Segala, "Time-bounded task-PIOAs: A framework for analyzing security protocols," in DISC'06, ser. LNCS, vol. 4167. Springer, 2006, pp. 238-253.

[32] P. Laud, "Secrecy types for a simulatable cryptographic library," in CCS'05. ACM, 2005, pp. 26-35.

[33] P. Laud and V. Vene, "A type system for computationally secure information flow," in FCT'05, ser. LNCS, vol. 3623. Springer, 2005, pp. 365-377.

[34] G. Smith and R. Alpízar, "Secure information flow with random assignment and encryption," in FMSE'06, 2006, pp. $33-43$.

[35] J. Courant, C. Ene, and Y. Lakhnech, "Computationally sound typing for non-interference: The case of deterministic encryption," in FSTTCS'07, ser. LNCS, vol. 4855. Springer, 2007, pp. 364-375.

[36] M. Backes and P. Laud, "Computationally sound secrecy proofs by mechanized flow analysis," in CCS'06. ACM, 2006, pp. 370-379.

[37] J. Courant, M. Daubignard, C. Ene, P. Lafourcade, and Y. Lakhnech, "Towards automated proofs for asymmetric encryption schemes in the random oracle model," in CCS'08. ACM, 2008, pp. 371-380.
[38] - "Automated proofs for asymmetric encryption," in Concurrency, Compositionality, and Correctness, ser. LNCS, vol. 5930. Springer, 2010, pp. 300-321.

[39] G. Barthe, B. Grégoire, and S. Zanella, "Formal certification of code-based cryptographic proofs," in POPL'09. ACM, 2009, pp. 90-101.

[40] G. Barthe, B. Grégoire, S. Heraud, and S. Z. Béguelin, "Formal certification of ElGamal encryption. A gentle introduction to CertiCrypt," in FAST 2008, ser. LNCS, vol. 5491. Springer, 2009, pp. 1-19.

[41] S. Z. Béguelin, B. Grégoire, G. Barthe, and F. Olmedo, "Formally certifying the security of digital signature schemes," in IEEE Symposium on Security and Privacy. IEEE, 2009, pp. 237-250.

[42] S. Z. Béguelin, G. Barthe, S. Heraud, B. Grégoire, and D. Hedin, "A machine-checked formalization of sigmaprotocols," in CSF'10. IEEE, 2010, pp. 246-260.

[43] V. Shoup, "Sequences of games: a tool for taming complexity in security proofs," Cryptology ePrint Archive, Report 2004/332, 2004, available at http://eprint.iacr.org/2004/332.

[44] M. Bellare and P. Rogaway, "The security of triple encryption and a framework for code-based game-playing proofs," in EUROCRYPT 2006, ser. LNCS, vol. 4004. Springer, 2006, pp. 409-426.

[45] D. Nowak, "A framework for game-based security proofs," in ICICS 2007, ser. LNCS, vol. 4861. Springer, 2007, pp. 319-333.

[46] - "On formal verification of arithmetic-based cryptographic primitives," in ICISC 2008, ser. LNCS, vol. 5461. Springer, 2008, pp. 368-382.

[47] R. Affeldt, D. Nowak, and K. Yamada, "Certifying assembly with formal cryptographic proofs: the case of BBS," in AVoCS'09, ser. Electronic Communications of the EASST, vol. 23, 2009.

[48] G. Barthe, B. Grégoire, S. Heraud, and S. Z. Béguelin, "Computer-aided security proofs for the working cryptographer," in CRYPTO 2011, 2011, to appear.

[49] B. Blanchet, "A computationally sound mechanized prover for security protocols," IEEE Transactions on Dependable and Secure Computing, vol. 5, no. 4, pp. 193-207, 2008.

[50] B. Blanchet and D. Pointcheval, "Automated security proofs with sequences of games," in CRYPTO 2006, ser. LNCS, vol. 4117. Springer, 2006, pp. 537-554.

[51] P. Laud, "Handling encryption in an analysis for secure information flow," in ESOP'03, ser. LNCS, vol. 2618. Springer, 2003, pp. 159-173.

[52] _ "Symmetric encryption in automatic analyses for confidentiality against active adversaries," in IEEE Symposium on Security and Privacy, 2004, pp. 71-85. 
[53] I. Tšahhirov and P. Laud, "Application of dependency graphs to security protocol analysis," in TGC'07, ser. LNCS, vol. 4912. Springer, 2007, pp. 294-311.

[54] P. Laud and I. Tšahhirov, "A user interface for a gamebased protocol verification tool," in FAST2009, ser. LNCS, vol. 5983. Springer, 2009, pp. 263-278.

[55] E. Bresson, O. Chevassut, and D. Pointcheval, "Security proofs for an efficient password-based key exchange," in CCS'03. ACM, 2003, pp. 241-250.

[56] S. M. Bellovin and M. Merritt, "Encrypted key exchange: Password-based protocols secure against dictionary attacks," in IEEE Symposium on Security and Privacy. IEEE, 1992, pp. $72-84$.

[57] B. Blanchet, "Automatically verified mechanized proof of one-encryption key exchange," in 25th IEEE Computer Security Foundations Symposium (CSF'12). IEEE, 2012, pp. 325-339.

[58] - "Automatically verified mechanized proof of oneencryption key exchange," Cryptology ePrint Archive, Report 2012/173, version 1, Apr. 2012, available at http://eprint.iacr. org/2012/173.

[59] M. Bellare and P. Rogaway, "The AuthA protocol for password-based authenticated key exchange," Mar. 2000, contributions to IEEE P1363. Available from http://grouper.ieee.org/groups/1363/.

[60] — - "Random oracles are practical: A paradigm for designing efficient protocols," in CCS'93. ACM, 1993, pp. 62-73.

[61] M. Bellare, D. Pointcheval, and P. Rogaway, "Authenticated key exchange secure against dictionary attacks," in EUROCRYPT 2000, ser. LNCS, vol. 1807. Springer, 2000, pp. $139-155$

[62] J.-S. Coron, "Security proof for partial-domain hash signature schemes," in CRYPTO 2002, ser. LNCS, vol. 2442. Springer, 2002, pp. 613-626.

[63] B. Blanchet, "Computationally sound mechanized proofs of correspondence assertions," in CSF'07. IEEE, 2007, pp. 97111, extended version available as ePrint Report 2007/128, http://eprint.iacr.org/2007/128.

[64] — "Composition theorems for CryptoVerif and application to TLS 1.3," in 31st IEEE Computer Security Foundations Symposium (CSF'18). IEEE, 2018, pp. 16-30.

[65] T. Y. C. Woo and S. S. Lam, "A semantic model for authentication protocols," in IEEE Symposium on Research in Security and Privacy, 1993, pp. 178-194.

[66] M. Abdalla, P.-A. Fouque, and D. Pointcheval, "Passwordbased authenticated key exchange in the three-party setting," IEE Proceedings Information Security, vol. 153, no. 1, pp. 27-39, 2006.

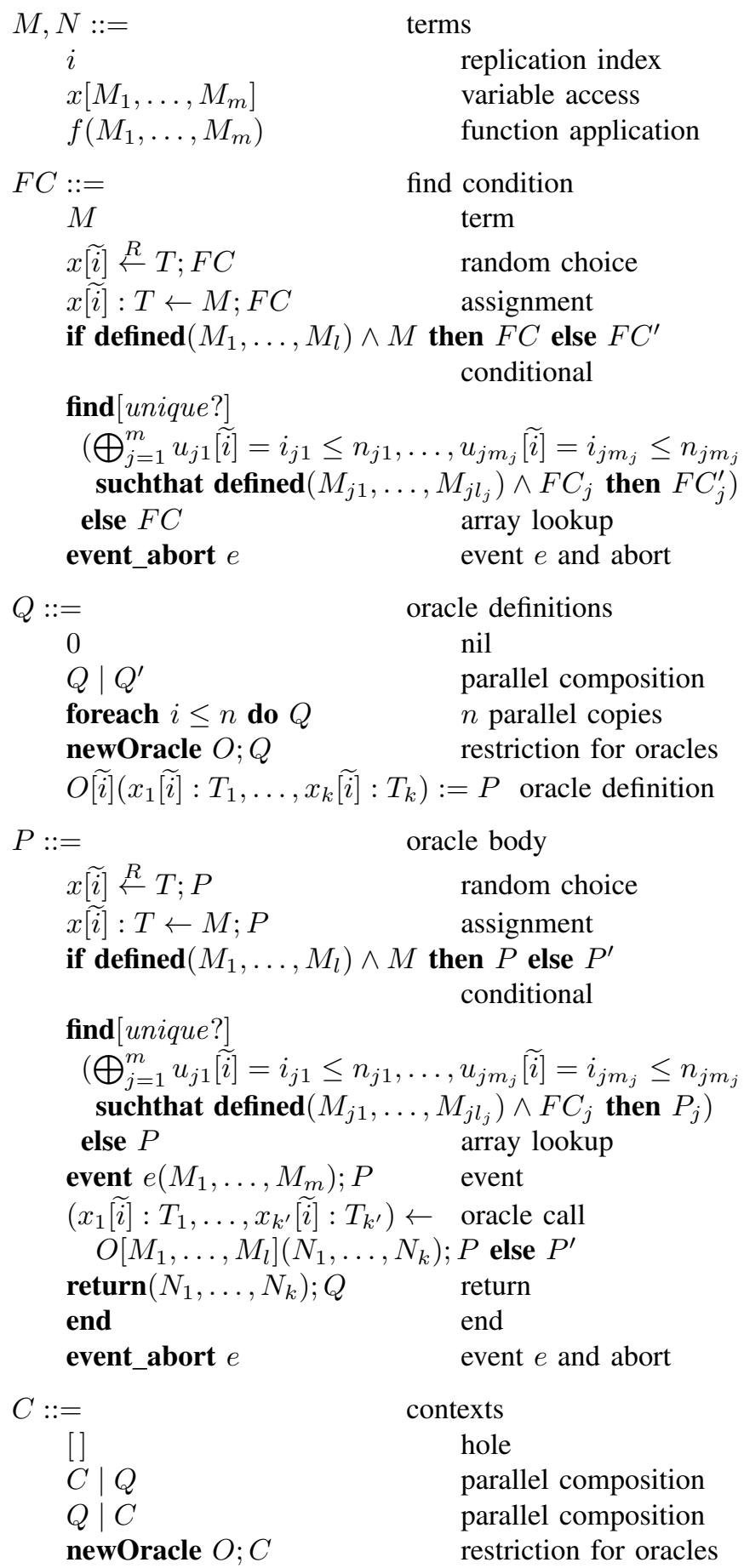

Figure 5. Syntax of the process calculus

\section{APPENDIX A.}

\section{BASIC DEFINITIONS AND PROPERTIES}

This appendix recalls and sometimes adapts the definitions of the basic concepts used by CryptoVerif. In CryptoVerif, games are represented in a process calculus. A similar calculus was presented in detail in [49], using channels 
instead of oracles and asymptotic security instead of exact security. The syntax of this calculus is recalled in Figure 5.

This calculus uses parameters, denoted by $n$, which represent integer values. This calculus also uses types, denoted by $T$, corresponding to subsets of bitstring $\cup\{\perp\}$ where bitstring is the set of all bitstrings and $\perp$ is a special symbol. We say that a type is large when its cardinal is large enough so that we can harmlessly eliminate collisions between random values of this type. Particular types are predefined: bool $=\{$ true, false $\}$, where false is 0 and true is 1 ; bitstring; bitstring ${ }_{\perp}=$ bitstring $\cup\{\perp\}$; $[1, n]$ where $n$ is a parameter. (We consider integers as bitstrings without leading zeroes.)

The calculus also uses function symbols $f$. Each function symbol comes with a type declaration $f: T_{1} \times \ldots \times T_{m} \rightarrow T$, and represents a function from $m$-tuples of bitstrings or $\perp$ in $T_{1} \times \ldots \times T_{m}$ to a bitstring or $\perp$. Particular functions are predefined, and some of them use the infix notation: $M=N$ for the equality test, $M \neq N$ for the inequality test (both taking two values of the same type $T$ and returning a value of type bool), $M \vee N$ for the boolean or, $M \wedge N$ for the boolean and, $\neg M$ for the boolean negation (taking and returning values of type bool).

In this calculus, terms represent computations on bitstrings. The replication index $i$ is an integer which serves in distinguishing different copies of a replicated process foreach $i \leq n$ do $Q$. (Replication indices are typically used as array indices.) The variable access $x\left[M_{1}, \ldots, M_{m}\right]$ returns the content of the cell of indices $M_{1}, \ldots, M_{m}$ of the $m$-dimensional array variable $x$. The function application $f\left(M_{1}, \ldots, M_{m}\right)$ returns the result of applying function $f$ to $M_{1}, \ldots, M_{m}$.

The calculus distinguishes two categories of processes: oracle definitions $Q$ consist of a set of definitions of oracles, while oracle bodies $P$ describe the content of an oracle definition. Oracle bodies perform some computations and return a result. After returning the result, they may define new oracles. (An oracle definition $Q$ follows the $\operatorname{return}\left(N_{1}, \ldots, N_{k}\right)$ instruction.)

The nil oracle definition 0 defines no oracle; $Q \mid Q^{\prime}$ is the parallel composition of $Q$ and $Q^{\prime}$ : it makes available both oracles defined in $Q$ and in $Q^{\prime}$; foreach $i \leq n$ do $Q$ represents $n$ copies of $Q$ in parallel, each with a different value of $i \in[1, n]$. The construct newOracle $O ; Q$ hides oracle $O$ outside $Q$ : oracle $O$ can be called only inside $Q$. The oracle definition $O \widetilde{[i]}\left(x_{1} \widetilde{[i]}: T_{1}, \ldots, x_{k} \widetilde{[i]}: T_{k}\right):=P$ defines an oracle $O$, taking arguments $x_{1}, \ldots, x_{k}$ of types $T_{1}, \ldots, T_{k}$ respectively, and computed as described in oracle body $P$, where $\widetilde{i}$ denotes a tuple $i_{1}, \ldots, i_{m}$.

The random choice $x \widetilde{[i]} \stackrel{R}{\leftarrow} T ; P$ chooses a new random number uniformly in $T$, stores it in $x[\widetilde{i}]$, and executes $P$. Function symbols represent deterministic functions, so all random numbers must be chosen by $x[\widetilde{i}] \stackrel{R}{\leftarrow} T$. The assignment $x \widetilde{[i]}: T \leftarrow M ; P$ stores the bitstring value of $M$ (which must be in $T$ ) in $x \widetilde{[i]}$ and executes $P$.

Next, we explain the array lookup find $\left(\bigoplus_{j=1}^{m} u_{j 1}[\widetilde{i}]=\right.$ $i_{j 1} \leq n_{j 1}, \ldots, u_{j m_{j}} \widetilde{[i]}=i_{j m_{j}} \leq n_{j m_{j}}$ suchthat $\operatorname{defined}\left(M_{j 1}, \ldots, M_{j l_{j}}\right) \wedge F C_{j}$ then $\left.P_{j}\right)$ else $P$. The order and array indices on tuples are taken component-wise, so for instance, $u_{j 1}[\widetilde{i}]=i_{j 1} \leq n_{\tilde{i}_{1}}, \ldots, u_{j m_{j}}[\tilde{i}]=i_{j m_{j}} \leq n_{j m_{j}}$ can be abbreviated $\left.\widetilde{u_{j}} \widetilde{[i}\right]=\widetilde{i_{j}} \leq \widetilde{n_{j}}$. A simple example is the following: find $u=i \leq n$ suchthat defined $(x[i]) \wedge x[i]=a$ then $P^{\prime}$ else $P$ tries to find an index $i$ such that $x[i]$ is defined and $x[i]=a$, and when such a $i$ is found, it stores it in $u$ and executes $P^{\prime}$; otherwise, it executes $P$. In other words, this find construct looks for the value $a$ in the array $x$, and when $a$ is found, it stores in $u$ an index such that $x[u]=a$. More generally, find $u_{1} \widetilde{[i]}=$ $i_{1} \leq n_{1}, \ldots, u_{m}[\tilde{i}]=i_{m} \leq n_{m}$ suchthat defined $\left(M_{1}\right.$, $\left.\ldots, M_{l}\right) \wedge F C$ then $P^{\prime}$ else $P$ tries to find values of $i_{1}, \ldots, i_{m}$ for which $M_{1}, \ldots, M_{l}$ are defined and $F C$ is true. In case of success, it stores these values in $u_{1}[\widetilde{i}]$, $\ldots, u_{m} \widetilde{[i]}$ respectively and executes $P^{\prime}$. In case of failure, it executes $P$. This is further generalized to $m$ branches: find $\left(\bigoplus_{j=1}^{m} u_{j 1} \widetilde{[i]}=i_{j 1} \leq n_{j 1}, \ldots, u_{j m_{j}} \widetilde{[i]}=i_{j m_{j}} \leq n_{j m_{j}}\right.$ suchthat defined $\left(M_{j 1}, \ldots, M_{j l_{j}}\right) \wedge F C_{j}$ then $\left.P_{j}\right)$ else $P$ tries to find a branch $j$ in $[1, m]$ such that there are values of $i_{j 1}, \ldots, i_{j m_{j}}$ for which $M_{j 1}, \ldots, M_{j l_{j}}$ are defined and $F C_{j}$ is true. In case of success, it stores these values in $u_{j 1}[\widetilde{i}]$, $\ldots, u_{j m_{j}}[\widetilde{i}]$ respectively and executes $P_{j}$. In case of failure for all branches, it executes $P$. More formally, it evaluates the conditions defined $\left(M_{j 1}, \ldots, M_{j l_{j}}\right) \wedge F C_{j}$ for each $j$ and each value of $i_{j 1}, \ldots, i_{j m_{j}}$ in $\left[1, n_{j 1}\right] \times \ldots \times\left[1, n_{j m_{j}}\right]$. If none of these conditions is true, it executes $P$. Otherwise, it chooses randomly with (almost) uniform probability one $j$ and one value of $i_{j 1}, \ldots, i_{j m_{j}}$ such that the corresponding condition is true, stores this value in $u_{j 1}[\widetilde{i}], \ldots, u_{j m_{j}} \widetilde{[i]}$, and executes $P_{j}$. (When the number of possibilities is not a power of 2, a probabilistic bounded-time Turing machine cannot choose these values exactly with uniform probability, but it can choose them with a probability distribution as close as we wish to uniform.) Optionally, one may add a [unique] modifier to find, represented in Figure 5 by [unique?]. When this modifier is present and there are several values of $j, i_{j 1}, \ldots, i_{j m_{j}}$ such that the corresponding condition is true, we execute the event NonUnique and abort the game. The find conditions $F C$ can themselves contain not only terms but also random choices, assignments, conditionals, array lookups, and event_abort.

The process if defined $\left(M_{1}, \ldots, M_{l}\right) \wedge M$ then $P$ else $P^{\prime}$ is syntactic sugar for find suchthat defined $\left(M_{1}, \ldots\right.$, $\left.M_{l}\right) \wedge M$ then $P$ else $P^{\prime}$.

The construct event $e\left(M_{1}, \ldots, M_{m}\right) ; P$ executes the event $e\left(M_{1}, \ldots, M_{m}\right)$, then executes $P$. Events just record that a certain program point has been reached, with certain values of the arguments of the event. They do not influence 
the execution of the rest of the process.

The oracle call $\left.\left(x_{1} \widetilde{[i]}: T_{1}, \ldots, x_{k^{\prime}} \widetilde{i}\right]: T_{k^{\prime}}\right) \leftarrow O\left[M_{1}\right.$, $\left.\ldots, M_{l}\right]\left(N_{1}, \ldots, N_{k}\right) ; P$ else $P^{\prime}$ calls oracle $O\left[M_{1}, \ldots\right.$, $\left.M_{l}\right]$ with arguments $N_{1}, \ldots, N_{k}$. When this oracle returns a result by $\operatorname{return}\left(N_{1}^{\prime}, \ldots, N_{k^{\prime}}^{\prime}\right)$, this result is stored in $x_{1}[\widetilde{i}]$, $\ldots, x_{k^{\prime}}[\widetilde{i}]$ and the process executes $P$. When the oracle $O\left[M_{1}, \ldots, M_{l}\right]$ terminates by end, the process executes $P^{\prime}$. (Returning a result by return corresponds to the normal termination of the oracle $O$, while terminating with end corresponds to abnormal termination.) Finally, the instruction event_abort $e$ executes event $e$ and aborts the game: the whole game terminates immediately and returns the special value abort.

To lighten notations, $\wedge$ true and $\operatorname{defined}() \wedge$ may be omitted in conditions of if and find. Moreover, else end, a trailing 0 , or a trailing end may be omitted. Types may be omitted when they can be inferred.

The current replication indices at a certain program point in a process are $i_{1}, \ldots, i_{m}$ when the considered program point is under foreach $i_{1} \leq n_{1}$ do $\ldots$ foreach $i_{m} \leq$ $n_{m}$ do . We abbreviate $x\left[i_{1}, \ldots, i_{m}\right]$ by $x$ when $i_{1}, \ldots, i_{m}$ are the current replication indices, but it should be kept in mind that this is only an abbreviation. Similarly, an oracle definition $O\left[i_{1}, \ldots, i_{m}\right](\ldots):=P$ under foreach $i_{1} \leq$ $n_{1} \ldots$ foreach $i_{m} \leq n_{m}$ is abbreviated $O(\ldots):=\bar{P}$. Variables and oracles defined under foreach must be indexed by the current replication indices: for example foreach $i_{1} \leq$ $n_{1}$ do $\ldots$ foreach $i_{m} \leq n_{m}$ do $\ldots x\left[i_{1}, \ldots, i_{m}\right]: T \leftarrow$ $M ; \ldots$ In the find construct, when the same identifier $u_{j k}$ is used for the variable $u_{j k}$ in $P_{j}$ and the replication index $i_{j k}$ in defined $\left(M_{j 1}, \ldots, M_{j l_{j}}\right) \wedge F C_{j}$, one abbreviates $u_{j k}[\widetilde{i}]=i_{j k} \leq n_{j k}$ into $u_{j k} \leq n_{j k}$.

We require some well-formedness invariants to guarantee that several definitions of the same oracle cannot be simultaneously available, that bitstrings are of their expected type, and that arrays are used properly (that each cell of an array is assigned at most once during execution, and that variables are accessed only after being initialized). Formally, we require the following invariants:

Invariant 1 (Single definition for oracles) The process $Q_{0}$ satisfies Invariant 1 if and only if

$1)$ in every definition of $O\left[i_{1}, \ldots, i_{m}\right]$ in $Q_{0}$, the indices $i_{1}, \ldots, i_{m}$ of $O$ are the current replication indices at that definition, and

2) two different definitions of the same oracle $O$ in $Q_{0}$ are in different branches of a find (or if).

Invariant 1 guarantees that each oracle is defined at most once for each value of its indices. (Indeed, item 2 shows that only one definition of each oracle can be available for given indices in each trace.)
Invariant 2 (Single definition for variables) The process $Q_{0}$ satisfies Invariant 2 if and only if

$1)$ in every definition of $x\left[i_{1}, \ldots, i_{m}\right]$ in $Q_{0}$, the indices $i_{1}, \ldots, i_{m}$ of $x$ are the current replication indices at that definition, and

2) two different definitions of the same variable $x$ in $Q_{0}$ are in different branches of a find (or if).

Similarly to the previous invariant, Invariant 2 guarantees that each variable is assigned at most once for each value of its indices.

Invariant 3 (Defined variables) The process $Q_{0}$ satisfies Invariant 3 if and only if every occurrence of a variable access $x\left[M_{1}, \ldots, M_{m}\right]$ in $Q_{0}$ is either

- syntactically under the definition of $x\left[M_{1}, \ldots, M_{m}\right]$ (in which case $M_{1}, \ldots, M_{m}$ are in fact the current replication indices at the definition of $x$ );

- or in a defined condition in a find construct;

- or in $F C_{j}^{\prime}$ in a process or find condition of the form find $\left(\bigoplus_{j=1}^{m^{\prime \prime}} \widetilde{u_{j}} \widetilde{[i]}=\widetilde{i_{j}} \leq \widetilde{n_{j}}\right.$ suchthat defined $\left(M_{j 1}^{\prime}\right.$, $\left.\ldots, M_{j l_{j}}^{\prime}\right) \wedge F C_{j}^{\prime}$ then $\ldots$ ) else ... where for some $k \leq l_{j}, x\left[M_{1}, \ldots, M_{m}\right]$ is a subterm of $M_{j k}^{\prime}$;

- or in $F C_{j}$ in a find condition of the form find $\left(\bigoplus_{j=1}^{m^{\prime \prime}} \widetilde{u_{j}} \widetilde{[i]}=\widetilde{i_{j}} \leq \widetilde{n_{j}}\right.$ suchthat defined $\left(M_{j 1}^{\prime}, \ldots\right.$, $\left.M_{j l_{j}}^{\prime}\right) \wedge F C_{j}^{\prime}$ then $F C_{j}$ ) else $F C$ where for some $k \leq l_{j}$, there is a subterm $N$ of $M_{j k}^{\prime}$ such that $\left.N\left\{\widetilde{u_{j}} \widetilde{[i}\right] / \widetilde{i_{j}}\right\}=x\left[M_{1}, \ldots, M_{m}\right]$.

- or in $P_{j}$ in a process of the form find $\left(\bigoplus_{j=1}^{m^{\prime \prime}} \widetilde{u_{j}} \widetilde{[i]}=\right.$ $\widetilde{i_{j}} \leq \widetilde{n_{j}}$ suchthat defined $\left(M_{j 1}^{\prime}, \ldots, M_{j l_{j}}^{\prime}\right) \wedge F C_{j}^{\prime}$ then $\left.P_{j}\right)$ else $P$ where for some $k \leqq l_{j}$, there is a subterm $N$ of $M_{j k}^{\prime}$ such that $\left.N\left\{\widetilde{u_{j}} \widetilde{i}\right] / \widetilde{i_{j}}\right\}=x\left[M_{1}, \ldots, M_{m}\right]$.

Invariant 3 guarantees that variables can be accessed only when they have been initialized. It checks that the definition of the variable access is either in scope (first item) or checked by the defined condition of a find (last three items). A variable access that does not correspond to the first item of Invariant 3 is called an array access. We furthermore require the following invariant.

Invariant 4 (Variables defined in find conditions) The process $Q_{0}$ satisfies Invariant 4 if and only if the variables defined in conditions of find have no array accesses.

This invariant did not appear in previous versions of the calculus because conditions of find were restricted to be terms.

We use a type system (see [49, Appendix A]) to check that bitstrings of the proper type are passed to each function and that array indices are used correctly.

Invariant 5 (Typing) The process $Q_{0}$ satisfies Invariant 5 if and only if it is well-typed. 
We require the adversary to be well-typed. This requirement does not restrict its computing power, because it can always define type-cast functions $f: T \rightarrow T^{\prime}$ to bypass the type system. Similarly, the type system does not restrict the class of protocols that we consider, since the protocol may contain type-cast functions. The type system just makes explicit which set of bitstrings may appear at each point of the protocol.

These invariants are checked by the prover for the initial game and preserved by all game transformations. We suppose that all games satisfy these invariants.

We use a context $C$ to represent an adversary. A context is a process with a hole. In this paper, we consider only evaluation contexts, generated by the grammar given at the bottom of Figure 5. A context $C$ is put around a process $Q$ by $C[Q]$. This construct means that $Q$ is put in parallel with some other process $Q^{\prime}$ contained in $C$, possibly hiding some oracles defined in $Q$, so that, when considering $C^{\prime}[C[Q]]$, $C^{\prime}$ cannot call these oracles.

A context $C$ is said to be acceptable for $Q$ with public variables $V$ if and only if the common variables of $C$ and $Q$ are in $V$, and $C[Q]$ satisfies the well-formedness invariants.

We name Ostart the oracle called to start the experiment. We denote by $\operatorname{Pr}[Q: D]$ the probability that, for some sequence of events $\mathcal{E}$ and bitstring $a$, when oracle $O \operatorname{start}()$ is called, $Q$ executes exactly the sequence $\mathcal{E}$, in the same order, and returns the result $a$, such that the algorithm $D(\mathcal{E}, a)$ returns true.

We denote by $\operatorname{var}(Q)($ resp. $\operatorname{var}(C))$ the set of variables of the process $Q$ (resp. context $C$ ).

The following lemma is a straightforward consequence of Definition 1:

Lemma 3 1) $Q \approx_{0}^{V} Q$.

2) $\approx_{p}^{V}$ is symmetric.

3) If $Q \approx_{p}^{V} Q^{\prime}$ and $Q^{\prime} \approx_{p^{\prime}}^{V} Q^{\prime \prime}$, then $Q \approx_{p+p^{\prime}}^{V} Q^{\prime \prime}$.

4) If $Q \approx_{p}^{V} Q^{\prime}$ and $C$ is a context acceptable for $Q$ and $Q^{\prime}$ with public variables $V$, then $C[Q] \approx \nabla_{p^{\prime}}^{V^{\prime}} C\left[Q^{\prime}\right]$, where $p^{\prime}\left(C^{\prime}, t_{D}\right)=p\left(C^{\prime}[C[]], t_{D}\right)$ and $V^{\prime} \subseteq V \cup$ $\operatorname{var}(C)$.

\section{APPENDIX B.}

The Computational Diffie-Hellman Assumption

\section{A. Proof of the Reduction}

First, let us rephrase the two games:

G1: the adversary is allowed to query

- for group elements, via oracles $\mathrm{OA}$ and $\mathrm{OB}$ : $\mathrm{OA}[i]()^{3}$ provides a $g^{a_{i}}$ element for a random $a_{i}$ (at most $n a$ queries), and $\mathrm{OB}[j]()$ provides a $g^{b_{j}}$ element for a random $b_{j}$ (at most $n b$ queries);

\footnotetext{
${ }^{3}$ The argument $[i]$ between brackets is implicit in the CryptoVerif syntax and corresponds to the replication index.
}

- for discrete logarithms, via oracles $\mathrm{Oa}$ and $\mathrm{Ob}$ : $\mathrm{Oa}[i]()$ outputs $a_{i}$ (at most \#Oa $\leq n a$ queries), $\mathrm{Ob}[j]()$ outputs $b_{j}$ (at most \#Ob $\leq n b$ queries);

- for exponentiations, via oracles ODHa and ODHb: $\mathrm{ODHa}[i](x)$ returns $g^{a_{i} x}$ (at most \#ODHa queries), ODHb $[j](x)$ returns $g^{b_{j} x}$ (at most \#ODHb queries);

- for Diffie-Hellman decisions, via oracles ODDHa and ODDHb: ODDHa $[i](m, j)$ checks whether $m=g^{a_{i} b_{j}}$ (at most naDDH queries for each $a$, at most \#ODDHa queries total), ODDHb $j](m$, i) checks whether $m=g^{a_{i} b_{j}}$ (at most $\mathrm{nbDDH}$ queries for each $b$, at most \#ODDHb queries total). We can thus combine them into $\operatorname{DDH}(m, i, j)$ queries (at most $q_{\text {ddh }}=\#$ ODDHa $+\# \mathrm{ODDHb} \leq$ $n a \cdot n b$ queries) which check whether $m=g^{a_{i} b_{j}}$.

G2: the adversary is allowed to query

- OA, OB, Oa, Ob, ODHa, and ODHb oracles, that answer as above;

- for $\operatorname{DDH}(m, i, j)$, Diffie-Hellman decisions, oracle. But in this game, the correct answer is given if either $a_{i}$ or $b_{j}$ has been asked before for an $\mathrm{Oa}$ or Ob query. Otherwise, the answer is 'false'.

We thus insist on the fact that the 2 games differ on $\operatorname{DDH}(m, i, j)$ Diffie-Hellman decisions queries, if neither $a_{i}$ nor $b_{j}$ has been asked before for an Oa or Ob query. In the first game, the answer is the correct one; in the second game, the answer is always 'false'.

Let us be given a CDH tuple $\left(X=g^{x}, Y=g^{y}\right)$ for which we want to compute $Z=g^{x y}$. And we provide a simulator $\mathcal{A}$ for these games:

- For the query $\mathrm{OA}[i]$, one chooses a random bit $\gamma_{i}$ with bias $p_{a}$ : with probability $p_{a}, \gamma_{i}=1$, and with probability $1-p_{a}, \gamma_{i}=0$. One also chooses a random scalar $\alpha_{i} \stackrel{R}{\leftarrow} \mathbb{Z}_{q}^{*}$. If $\gamma_{i}=1$, set $A_{i}=X^{\alpha_{i}}$ (and thus we have $a_{i}=\alpha_{i} x$ but the simulator cannot compute $a_{i}$ because it does not know $x$ ). If $\gamma_{i}=0$, set $a_{i}=\alpha_{i}$ and $A_{i}=g^{\alpha_{i}}$.

- For the query $\mathrm{OB}[j]$, one chooses a random bit $\delta_{j}$ with bias $p_{b}$ : with probability $p_{b}, \delta_{j}=1$, and with probability $1-p_{b}, \delta_{j}=0$. One also chooses a random scalar $\beta_{j} \stackrel{R}{\leftarrow} \mathbb{Z}_{q}^{*}$. If $\delta_{j}=1$, set $B_{j}=Y^{\beta_{j}}$ (and thus $b_{j}=\beta_{j} y$ ). If $\delta_{j}=0$, set $b_{j}=\beta_{j}$ and $B_{j}=g^{\beta_{j}}$.

- For the query $\mathrm{Oa}[i]$, with probability $1-p_{a}, \gamma_{i}=0$, and then the correct answer $\alpha_{i}$ can be sent. However, with probability $p_{a}$ on the current $a$, the simulation fails.

- For the query $\mathrm{Ob}[j]$, with probability $1-p_{b}, \delta_{j}=0$, and then the correct answer $\beta_{j}$ can be sent. However, with probability $p_{b}$ on the current $b$, the simulation fails.

- For the query $\mathrm{ODHa}[i](x)$, return $A_{i}^{x}$.

- For the query ODHb $[j](x)$, return $B_{j}^{x}$.

Since there are at most \#Oa Oa queries and $\# \mathrm{Ob} \mathrm{Ob}$ queries, with probability at least $\left(1-p_{a}\right)^{\# \mathrm{Oa}}\left(1-p_{b}\right)^{\# \mathrm{Ob}}$, 
the simulation does not fail, and is perfectly indistinguishable from the real oracles OA, OB, Oa, Ob, ODHa, and ODHb. Let us now consider the DDH Diffie-Hellman Decision queries, and the simulation of the answers when no failure happens during $\mathrm{Oa}$ and $\mathrm{Ob}$ simulation: for any $\operatorname{DDH}(m, i, j)$,

- if one of the $a_{i}$ or $b_{j}$ has been asked an Oa or Ob-query (and did not lead to a failure), which means that either $\gamma_{i}=0$ or $\delta_{j}=0$, then one can either test whether $m=\left(g^{b_{j}}\right)^{\alpha_{i}}$ or not, or whether $m=\left(g^{a_{i}}\right)^{\beta_{j}}$ or not, and provide the correct answer. This leads to a perfect simulation of the DDH oracle;

- otherwise, one can safely answer 'false', which leads to a perfect simulation of the DDH oracle in the second game. However, it differs from the first game oracle if $m=g^{a_{i} b_{j}}$.

As a consequence, the two games differ if for one DDH query, $m=g^{a_{i} b_{j}}$ but neither $a_{i}$ or $b_{j}$ has been asked for an $\mathrm{Oa}$ or $\mathrm{Ob}$ query. In this case, with probability $p_{a} p_{b}$, both $\gamma_{i}=1$ and $\delta_{j}=1$ :

$$
m=g^{a_{i} b_{j}}=g^{\alpha_{i} x \beta_{j} y}=Z^{\alpha_{i} \beta_{j}} .
$$

If the two above games differ with probability $\varepsilon$, then such a critical DDH query happens with probability $\varepsilon$, since our simulation is perfectly indistinguishable from the second game and such a critical query is the unique event that makes the two games different.

Let us randomly choose $k$ between 1 and $q_{\mathrm{ddh}}$, and bet that the $k$-th DDH query is the first critical one, which is true with probability $\varepsilon / q_{\text {ddh }}$. Furthermore, with probability $\left(1-p_{a}\right)^{\# \mathrm{Oa}}\left(1-p_{b}\right)^{\# \mathrm{Ob}} p_{a} p_{b}$ the simulation did not fail and the critical DDH query leads to the expected $Z$ value, by computing $Z=m^{1 /\left(\alpha_{i} \beta_{j}\right)}$. This means that our simulator $\mathcal{A}$ achieves $\operatorname{Succ}_{\mathbb{G}}^{\text {cdh }}(\mathcal{A}) \geq\left(1-p_{a}\right)^{\# \mathrm{Oa}}\left(1-p_{b}\right)^{\# \mathrm{Ob}} p_{a} p_{b} \varepsilon / q_{\mathrm{ddh}}$, and is bounded by $\operatorname{Succ}_{\mathbb{G}}^{\text {cdh }}\left(t+\left(n a+n b+q_{\text {ddh }}^{\prime}+\# \mathrm{ODHa}+\right.\right.$ \#ODHb) $\tau_{\text {exp }}$ ) where $q_{\mathrm{ddh}}^{\prime}=0$ if $\mathrm{Oa}$ and $\mathrm{Ob}$ are never called $(\# \mathrm{Oa}=0$ and $\# \mathrm{Ob}=0)$ and $q_{\mathrm{ddh}}^{\prime}=q_{\mathrm{ddh}}=\# \mathrm{ODDHa}+$ \#ODDHb otherwise:

$$
\begin{aligned}
& \varepsilon \leq q_{\mathrm{ddh}} \times \frac{1}{p_{a}\left(1-p_{a}\right)^{\# \mathrm{Oa}}} \times \frac{1}{p_{b}\left(1-p_{b}\right)^{\# \mathrm{Ob}}} \times \\
& \operatorname{Succ}_{\mathbb{G}}^{\mathrm{cdh}}\left(t+\left(n a+n b+q_{\mathrm{ddh}}^{\prime}+\# \mathrm{ODHa}+\# \mathrm{ODHb}\right) \tau_{\exp }\right)
\end{aligned}
$$

Two cases appear for the function $x \mapsto 1 /\left(x(1-x)^{n}\right)$ :

- if $n=0$, then the minimum is 1 , for $x=1$;

- if $n \geq 1$, then the minimum is reached for $x=1 /(n+$ $1)$, and its value is $\frac{(n+1)^{n+1}}{n^{n}}$.

Let

$$
h(0)=1, h(n)=\frac{(n+1)^{n+1}}{n^{n}} \text { for } n \geq 1
$$

By choosing the right value of $x$, we have $1 /\left(x(1-x)^{n}\right) \leq$ $h(n)$. We are going to show that $h(n) \leq 3 n+1$ for $n \geq 0$.
For information, we have

$$
\frac{h(n)}{n}=\left(1+\frac{1}{n}\right)^{n+1} \rightarrow e
$$

when $n$ tends to $+\infty$ and we have

$$
e n \leq h(n) \leq \max (1,4 n)
$$

and

$$
h(n) \leq e(n+1)
$$

The first inequality holds because $\frac{h(n)}{n}=\left(1+\frac{1}{n}\right)^{n+1}$ is monotonically decreasing, tends to $e$ when $n$ tends to $+\infty$, and evaluates to 4 for $n=1$. Indeed, let $l(n)=$ $\ln \left(\left(1+\frac{1}{n}\right)^{n+1}\right)=(n+1) \ln \left(1+\frac{1}{n}\right)$. We have $l^{\prime}(n)=$ $\ln \left(1+\frac{1}{n}\right)-\frac{1}{n} \leq 0$, so $l$ is monotonically decreasing.

The second inequality holds because $\frac{h(n)}{n+1}=\left(1+\frac{1}{n}\right)^{n}$ is monotonically increasing and tends to $e$ when $n$ tends to $+\infty$. Indeed, let $l(n)=\ln \left(\left(1+\frac{1}{n}\right)^{n}\right)=n \ln \left(1+\frac{1}{n}\right)$. We have $l^{\prime}(n)=\ln \left(1+\frac{1}{n}\right)-\frac{1}{n+1}$ and $l^{\prime \prime}(n)=\frac{-\frac{1}{n^{2}}}{1+\frac{1}{n}}+\frac{1}{(n+1)^{2}}=$ $-\frac{1}{n(n+1)^{2}}<0$ for $n>0$ so $l^{\prime}$ is monotonically decreasing and $l^{\prime}(n)$ tends to 0 when $n$ tends to $+\infty$. Therefore, $l^{\prime}(n)>$ 0 for all $n>0$, so $l$ is monotonically increasing.

We could use one of the bounds given by these two inequalities. However, the bound given by the first inequality is always larger than $3 n+1$. The bound given by the second inequality is best for large $n$, however it is not precise for small $n$. We believe that the bound $3 n+1$ yields a better compromise for all $n$. Let us show this bound. Let

We just have to show that, for all $n \geq 1, h(n) \leq 3 n+1$.

$$
\begin{aligned}
g(x) & =\ln \left(\frac{3 x+1}{h(x)}\right) \\
& =\ln (3 x+1)+x \ln (x)-(x+1) \ln (x+1)
\end{aligned}
$$

It is enough to show that for all $x \geq 1, g(x) \geq 0$. We have $g(1)=0$. Hence it is sufficient to show that $g$ is monotonically increasing. We have

$$
g^{\prime}(x)=\frac{3}{3 x+1}+\ln (x)-\ln (x+1)
$$

and

$$
\begin{aligned}
g^{\prime \prime}(x) & =-\frac{9}{(3 x+1)^{2}}+\frac{1}{x}-\frac{1}{x+1} \\
& =\frac{-9 x(x+1)+(3 x+1)^{2}}{(3 x+1)^{2} x(x+1)} \\
& =\frac{1-3 x}{(3 x+1)^{2} x(x+1)}<0
\end{aligned}
$$

for $x \geq 1$. Hence, $g^{\prime}$ is monotonically decreasing. Furthermore,

$$
g^{\prime}(x)=\frac{3}{3 x+1}+\ln \left(\frac{x}{x+1}\right)
$$


tends towards 0 when $x$ tends to $+\infty$, so $g^{\prime}(x) \geq 0$. Therefore, $g$ is monotonically increasing, and the desired result is proved.

Additionally, let us evaluate how precise the bound is. For that, we evaluate the limit of $g(x)$ when $x$ tends to $+\infty$. We have

$$
\begin{aligned}
g(x) & =\ln \left(\frac{3 x+1}{x}\right)-(x+1) \ln \left(\frac{x+1}{x}\right) \\
& \sim \ln (3)-x \frac{1}{x} \\
& \rightarrow \ln (3)-1
\end{aligned}
$$

when $x$ tends to $+\infty$. Therefore, for all $x \geq 1,0 \leq g(x)<$ $\ln (3)-1$, hence for all $n \geq 0$,

$$
1 \leq \frac{3 n+1}{h(n)}<\frac{3}{e}<1.104
$$

Hence, the loss coming from the replacement of $h(n)$ with $3 n+1$ is less than $10.4 \%$. That is remarkably precise given that the formula $3 n+1$ is much simpler than $h(n)$.

Hence we get $1 /\left(1-p_{a}\right)^{\# \mathrm{Oa}} p_{a} \leq 3 \# \mathrm{Oa}+1$ and $1 /(1-$ $\left.p_{b}\right)^{\# \mathrm{Ob}} p_{b} \leq 3 \# \mathrm{Ob}+1$, so

$\varepsilon \leq(\# \mathrm{ODDHa}+\# \mathrm{ODDHb})(3 \# \mathrm{Oa}+1)(3 \# \mathrm{Ob}+1) \times$

$\operatorname{Succ}_{\mathbb{G}}^{\text {cdh }}\left(t+\left(n a+n b+q_{\mathrm{ddh}}^{\prime}+\# \mathrm{ODHa}+\# \mathrm{ODHb}\right) \tau_{\exp }\right)$.

\section{B. Additional Modeling}

In the CryptoVerif implementation, the equivalence $L_{3} \approx_{p_{3}} R_{3}$ contains three additional indications, which we have omitted in Figure 4:

- In $L_{3}$, the oracles $\mathrm{Oa}$ and $\mathrm{Ob}$ are marked with the integer "[10]" and ODHa and ODHb are marked with "[2]". CryptoVerif tries to use oracles with the lowest mark first. (No mark means [0].) Here, the goal is to make sure that $g^{a}$ is obtained by calling OA and not by calling $\mathrm{Oa}$ to obtain $a$ and then computing $g^{a}$ (and similarly for $m=g^{a b}$ obtained by calling ODDHa or $\mathrm{ODDHb}$ rather than $\mathrm{Oa}$ and $\mathrm{Ob}$ or $\mathrm{ODHa}$ and $\mathrm{Ob}$ ). Indeed, if $\mathrm{Oa}$ is called, then the $\mathrm{CDH}$ assumption on that $a$ can no longer be applied. Therefore, CryptoVerif should use OA, OB, ODDHa, ODDHb, ODHa, or $\mathrm{ODHb}$ rather than $\mathrm{Oa}$ or $\mathrm{Ob}$ where possible, hence we give $\mathrm{Oa}$ and $\mathrm{Ob}$ a higher mark than the other oracles. Similarly, it should use ODDHa or ODDHb rather than ODHa or ODHb where possible, so we give ODHa and ODHb a higher mark than ODDHa or ODDHb.

- In $L_{3}$, the oracle ODDHa is marked [useful_change]. This prevents the application of the transformation of $L_{3}$ into $R_{3}$ when the initial game can be encoded without calling ODDHa. Indeed, the transformation has a useful effect only when ODDHa or ODDHb are called and, by symmetry, we can require that ODDHa is called.
- The equivalence property $L_{3} \quad \approx_{p_{3}} R_{3}$ is marked "[computational]" and the random choices $a \stackrel{R}{\leftarrow} Z$ and $b \stackrel{R}{\leftarrow} Z$ are marked "[unchanged]" in $R_{3}$. These annotations mean that we actually have a property slightly stronger than $L_{3} \approx_{p_{3}} R_{3}$ : the adversary can still not distinguish the games even if $a$ and $b$ are used inside events, in addition to the oracles provided in $L_{3}$ and $R_{3}$. This property holds because the proof of Appendix B-A shows that, using the same values of $a$ and $b$ in $L_{3}$ and $R_{3}$ (hence $a$ and $b$ are "[unchanged]"), the probability that the oracles of $L_{3}$ resp. $R_{3}$ return different values is bounded by $p_{3}$. From this property, we can show the indistinguishability of $L_{3}$ and $R_{3}$ with additional events using $a$ and $b$, since the events will be the same on both sides and the games can be distinguished only when the oracles of $L_{3}$ resp. $R_{3}$ return different values, which is bounded by $p_{3}$. (A cryptographic property is said to be "computational" when it holds except when some bad event happens, here the oracles of $L_{3}$ resp. $R_{3}$ return different values. Decisional properties such as the decisional DiffieHellman assumption are inherently indistinguishability properties and cannot be expressed using such a bad event.)

Moreover, we use the following properties:

collision $x \stackrel{R}{\leftarrow} Z ; \forall y: Z, \forall z: Z$;

$\operatorname{return}(\operatorname{mult}(x, y)=z) \approx_{1 /|Z|} \operatorname{return}($ false $)$

if $y$ independent-of $x \wedge z$ independent-of $x$.

collision $x \stackrel{R}{\leftarrow} Z ; y \stackrel{R}{\leftarrow} Z ; \forall z: Z$;

$\operatorname{return}(\operatorname{mult}(x, y)=z) \approx_{1 /|Z|} \operatorname{return}($ false $)$

if $z$ independent-of $x \vee z$ independent-of $y$.

collision $x \stackrel{R}{\leftarrow} Z ; y \stackrel{R}{\leftarrow} Z ;[$ random_choices_may_be_equal $]$

$\forall z: Z ; \operatorname{return}(\operatorname{mult}(x, y)=z) \approx_{2 /|Z|} \operatorname{return}($ false $)$

if $z$ independent-of $x \vee z$ independent-of $y$.

collision $x_{1} \stackrel{R}{\leftarrow} Z ; y_{1} \stackrel{R}{\leftarrow} Z ; x_{2} \stackrel{R}{\leftarrow} Z ; y_{2} \stackrel{R}{\leftarrow} Z$;

[random_choices_may_be_equal]

$\operatorname{return}\left(\operatorname{mult}\left(x_{1}, y_{1}\right)=\operatorname{mult}\left(x_{2}, y_{2}\right)\right) \approx_{2 /|Z|} \operatorname{return}($ false $)$

if $\left(x_{1}\right.$ independent-of $x_{2} \vee y_{1}$ independent-of $\left.y_{2}\right) \wedge$

$\left(x_{1}\right.$ independent-of $y_{2} \vee y_{1}$ independent-of $\left.x_{2}\right)$.

collision $x \stackrel{R}{\leftarrow} Z ; \forall X: G, \forall Y: G$;

$\operatorname{return}(\exp (X, x)=Y) \approx_{1 /|Z|} \operatorname{return}($ false $)$

if $X$ independent-of $x \wedge Y$ independent-of $x$

The statement (13) means that, if $x$ is uniformly random in $Z$ and $y$ and $z$ are independent of $x$, then the equality $\operatorname{mult}(x, y)=z$ is false except in cases of probability $1 /|Z|$. 
Indeed, the collision $x y=z$ happens when $x=z / y$ ( $y$ is invertible since we work in $\mathbb{Z}_{q}^{*}$ with $q$ prime). Since $z / y$ is independent of $x$, this has probability $1 /|Z|$ to happen.

The statement (14) means that, if $x$ and $y$ are uniformly random in $Z$ and independent, and $z$ is independent of $x$ or of $y$, then the equality mult $(x, y)=z$ is false except in cases of probability $1 /|Z|$. Its proof is similar to the one of (13), by distinguishing whether $z$ is independent of $x$ or of $y$.

In (15), the annotation [random_choices_may_be_equal] means that the statement holds when the random choices are either equal or independent of each other. Grouping these cases together avoids that CryptoVerif has two determine whether random choices are independent or equal, which may depend on values of indices at runtime. When $x$ and $y$ are independent, (15) is a consequence of (13). When $x$ and $y$ are equal, (15) says that for uniformly random $x$ and $z$ independent of $x$, the probability that $x^{2}=z$ is at most $2 /|Z|$. Indeed, in $\mathbb{Z}_{q}^{*}$, half of the elements are square, and each square has 2 square roots. If $z$ is not a square, the equality $x^{2}=z$ is false. If $z$ is a square $z=x^{\prime 2}$, then $x^{2}=z$ if and only if $x^{2}=x^{\prime 2}$ if and only if $x=x^{\prime}$ or $x=-x^{\prime}$, so with probability $2 /|Z|$.

In (16), the condition $\left(x_{1}\right.$ independent-of $x_{2} \vee$ $y_{1}$ independent-of $\left.y_{2}\right) \wedge\left(x_{1}\right.$ independent-of $y_{2} \vee$ $y_{1}$ independent-of $x_{2}$ ) yields 4 cases:

1) $x_{1}$ is independent of $x_{2}$ and $x_{1}$ is independent of $y_{2}$. So $x_{2}$ and $y_{2}$ are independent of $x_{1}$, so mult $\left(x_{2}, y_{2}\right)$ is independent of $x_{1}$ : (16) is a consequence of (15) with $z=\operatorname{mult}\left(x_{2}, y_{2}\right)$.

2) $x_{1}$ is independent of $x_{2}$ and $y_{1}$ is independent of $x_{2}$. So $x_{1}$ and $y_{1}$ are independent of $x_{2}$, so mult $\left(x_{1}, y_{1}\right)$ is independent of $x_{2}$ : (16) is a consequence of (15) with $z=\operatorname{mult}\left(x_{1}, y_{1}\right)$.

3) $y_{1}$ is independent of $y_{2}$ and $x_{1}$ is independent of $y_{2}$. So $x_{1}$ and $y_{1}$ are independent of $y_{2}$, so mult $\left(x_{1}, y_{1}\right)$ is independent of $y_{2}$ : (16) is a consequence of (15) with $z=\operatorname{mult}\left(x_{1}, y_{1}\right)$.

4) $y_{1}$ is independent of $y_{2}$ and $y_{1}$ is independent of $x_{2}$. So $x_{2}$ and $y_{2}$ are independent of $y_{1}$, so mult $\left(x_{2}, y_{2}\right)$ is independent of $y_{1}$ : (16) is a consequence of (15) with $z=\operatorname{mult}\left(x_{2}, y_{2}\right)$.

The statement (17) means that, when $x$ is uniformly random in $Z$ and $X$ and $Y$ in $G$ are independent of $x$, the probability of $X^{x}=Y$ is at most $1 /|Z|$. Indeed, $X$ is a generator of the group $\mathbb{G}$, so $Y=X^{y}$ for some $y \in Z$ independent of $x$. The equality $X^{x}=Y=X^{y}$ holds only when $x=y$ (modulo $q$ ) with $y$ independent of $x$, so this has probability $1 /|Z|$ of happening.

The formulas (4), (5), and (13) to (17) allow CryptoVerif to simplify equalities between exponentials.
APPENDIX C.

\section{PROOFS OF LEMMAS 1 AND 2}

Proof of Lemma 1: For Property 1, if $C\left[G^{\prime}\right]$ does not execute $e$ and $D$ returns false, then $C[G]$ behaves like $C\left[G^{\prime}\right]$ since $C[G]$ and $C\left[G^{\prime}\right]$ differ only when $e$ is executed, so $D$ also returns false on the execution of $C[G]$. Hence $\operatorname{Pr}\left[C\left[G^{\prime}\right]: \neg(D \vee e)\right] \leq \operatorname{Pr}[C[G]: \neg D]$. Property 1 follows.

For Property 2, if $C[G]$ satisfies $D=\left(D_{0} \wedge\right.$ $\neg$ NonUnique) $\vee e_{1} \vee \ldots \vee e_{n}$, then $C[G]$ does not execute NonUnique: this is clear by definition when $C[G]$ satisfies $D_{0} \wedge \neg$ NonUnique; when it satisfies $e_{1} \vee \ldots \vee e_{n}, C[G]$ also does not execute NonUnique because one aborts immediately after the events $e_{1}, \ldots, e_{n}$ and after NonUnique, so these events are pairwise incompatible. Hence, $G^{\prime}$ behaves as $G$ when $C[G]$ satisfies $D$. Hence $\operatorname{Pr}[C[G]: D] \leq \operatorname{Pr}\left[C\left[G^{\prime}\right]\right.$ : $D]$.

Property 3 is an immediate consequence of Definition 1. Property 4 is obvious.

Proof of Lemma 2: Property 1: By Lemma 1 (Property 1$), \operatorname{Pr}\left[C\left[G \mid R_{x}\right]: \mathrm{S} \vee D\right] \leq \operatorname{Pr}\left[C\left[G^{\prime} \mid R_{x}\right]: \mathrm{S} \vee D \vee e\right]$ Moreover, if $C\left[G^{\prime} \mid R_{x}\right]$ executes $\overline{\mathrm{S}}$ or NonUnique, $C\left[G^{\prime} \mid\right.$ $R_{x}$ ] does not execute $e$ (since we abort immediately after $\overline{\mathrm{S}}$, NonUnique, and $e$ ), so $C\left[G \mid R_{x}\right]$ behaves like $C\left[G^{\prime} \mid R_{x}\right]$, thus $C\left[G \mid R_{x}\right]$ also executes $\overline{\mathrm{S}}$ or NonUnique. Therefore, $\operatorname{Pr}\left[C\left[G^{\prime} \mid R_{x}\right]: \overline{\mathrm{S}} \vee\right.$ NonUnique $] \leq \operatorname{Pr}\left[C\left[G \mid R_{x}\right]:\right.$ $\overline{\mathrm{S}} \vee$ NonUnique]. Property 1 follows.

Property 2: We have

$$
\begin{aligned}
& \operatorname{Pr}\left[C\left[G \mid R_{x}\right]: \neg(\overline{\mathrm{S}} \vee \text { NonUnique })\right] \\
& \quad \leq \operatorname{Pr}\left[C\left[G^{\prime} \mid R_{x}\right]: \neg(\overline{\mathrm{S}} \vee \text { NonUnique })\right]
\end{aligned}
$$

since, when $G$ does not execute $\overline{\mathrm{S}}$ nor NonUnique, a fortiori, it does not execute NonUnique, so $G^{\prime}$ behaves as $G$. Therefore,

$$
\begin{aligned}
& -\operatorname{Pr}\left[C\left[G \mid R_{x}\right]: \overline{\mathrm{S}} \vee \text { NonUnique }\right] \\
& \quad \leq-\operatorname{Pr}\left[C\left[G^{\prime} \mid R_{x}\right]: \overline{\mathrm{S}} \vee \text { NonUnique }\right]
\end{aligned}
$$

Moreover,

$$
\operatorname{Pr}\left[C\left[G \mid R_{x}\right]: \mathrm{S} \vee D\right] \leq \operatorname{Pr}\left[C\left[G^{\prime} \mid R_{x}\right]: \mathrm{S} \vee D\right]
$$

since, when $G$ executes $\mathrm{S}$ or an event in $D$, it does not execute NonUnique (because one aborts immediately after $\mathrm{S}$, the events in $D$, and NonUnique), so $G^{\prime}$ behaves as $G$. Property 2 follows.

Property 3: Let $t=\max \left(t_{\mathrm{S} \vee D}, t_{\overline{\mathrm{S}} \vee \text { NonUnique }}\right)$. By definition of indistinguishability,

$$
\begin{aligned}
& \operatorname{Pr}\left[C\left[G \mid R_{x}\right]: \mathrm{S} \vee D\right] \\
& \quad \leq p\left(C\left[[] \mid R_{x}\right], t\right)+\operatorname{Pr}\left[C\left[G^{\prime} \mid R_{x}\right]: \mathrm{S} \vee D\right]
\end{aligned}
$$

and

$$
\begin{aligned}
& \operatorname{Pr}\left[C\left[G^{\prime} \mid R_{x}\right]: \overline{\mathrm{S}} \vee \text { NonUnique }\right] \\
& \quad \leq p\left(C\left[[] \mid R_{x}\right], t\right)+\operatorname{Pr}\left[C\left[G \mid R_{x}\right]: \overline{\mathrm{S}} \vee \text { NonUnique }\right]
\end{aligned}
$$


So

$$
\operatorname{Adv}_{G}^{\text {Secrecy }}(C, D) \leq 2 p\left(C\left[[] \mid R_{x}\right], t\right)+\operatorname{Adv}_{G^{\prime}}^{\text {Secrecy }}(C, D)
$$

Property 4 is obvious.

Property 5: When CryptoVerif proves the secrecy of $x$ in game $G$, it shows that only a certain set of variables depends on value of $x$, but the output messages and the control-flow do not depend on $x$. Hence an execution of $C\left[G \mid R_{x}\right]$ that calls oracle $O^{\prime}$ (defined in Definition 2) in which $b=1$ sends the same messages and has the same value of $b^{\prime}$, as the executions with the same random choices except that $b=0, y[i]$ has the value of $x[u[i]]$, and $x$ takes any value. The execution with $b=1$ executes $\mathrm{S}$ if and only if the executions with $b=0$ execute $\overline{\mathrm{S}}$.

Therefore, $\operatorname{Pr}\left[C\left[G \mid R_{x}\right]: \mathrm{S}\right]=\operatorname{Pr}\left[C\left[G \mid R_{x}\right]: \overline{\mathrm{S}}\right]$. Since we abort immediately after each event $\mathrm{S}, e_{1}, \ldots, e_{n}, \mathrm{~S}$ is incompatible with $e_{1}, \ldots, e_{n}$, so

$$
\begin{aligned}
& \operatorname{Pr}\left[C\left[G \mid R_{x}\right]: \mathrm{S} \vee D\right] \\
& \quad=\operatorname{Pr}\left[C\left[G \mid R_{x}\right]: \mathrm{S}\right]+\operatorname{Pr}\left[C\left[G \mid R_{x}\right]: D\right]
\end{aligned}
$$

Hence

$$
\begin{aligned}
& \operatorname{Adv}_{G}^{\text {Secrecy }}(C, D) \\
& =\operatorname{Pr}\left[C\left[G \mid R_{x}\right]: \mathrm{S}\right]+\operatorname{Pr}\left[C\left[G \mid R_{x}\right]: D\right] \\
& \quad-\operatorname{Pr}\left[C\left[G \mid R_{x}\right]: \overline{\mathrm{S}} \vee \text { NonUnique }\right] \\
& \leq \operatorname{Pr}\left[C\left[G \mid R_{x}\right]: D\right]
\end{aligned}
$$

\section{APPENDIX D.}

\section{IMPROVED COMPUTATION OF PROBABILITIES FOR THE MANUAL PROOF OF OEKE}

To illustrate the use of our improved computation of probabilities of Section IV-C, we apply it to the manual proof of OEKE [55]. We just recall the structure of the proof and the computation of probabilities, and refer the reader to [55] for details of the proof. Let us consider the proof of semantic security [55, Section 3.2]. The proof starts from a game $G_{0}$ that represents the OEKE protocol, in which we define a test-query that returns either the session key or a random value, depending on the value of a bit $b$, an event $S$ executed when the adversary guesses $b$ correctly, and an event $\bar{S}$ executed when the adversary guesses the wrong value of $b$. The probability that the adversary $C$ guesses $b$ correctly in $G_{0}$ is $\operatorname{Pr}\left[C\left[G_{0}\right]: \mathrm{S}\right]$, the probability that it guesses the wrong value of $b$ is $\operatorname{Pr}\left[C\left[G_{0}\right]: \overline{\mathrm{S}}\right]$ and the advantage of the adversary $C$ in distinguishing the session key from a random key is $\operatorname{Adv}_{\text {oeke }}^{\text {ake }}(C)=\operatorname{Pr}\left[C\left[G_{0}\right]: \mathrm{S}\right]-\operatorname{Pr}\left[C\left[G_{0}\right]: \overline{\mathrm{S}}\right]$.

The proof then proceeds as follows. The game $G_{0}$ is transformed into games $G_{1}, G_{2}, G_{3}$, by eliminating collisions, such that

$$
G_{0} \approx \frac{q_{\mathcal{E}}^{2}}{2(q-1)} G_{1} \approx_{\frac{q_{S} q_{\mathcal{E}}}{q-1}} G_{2} \approx_{\frac{2 q_{\mathcal{E}}^{2}+q_{S}^{2}}{2(q-1)}+\frac{q_{h}^{2}}{2^{l_{1}+1}}} G_{3}
$$

where $q_{S}$ is the number of involved server instances, $q_{\mathcal{E}}$ is the number of encryption/decryption queries, $q_{h}$ is the number of hash queries, $l_{1}$ is the length of the output of $\mathcal{H}_{1}, q$ is the order of $\mathbb{G}$.

Then $G_{3}$ is transformed into $G_{4}$ by inserting event Encrypt. Game $G_{4}$ is transformed into $G_{5}$ by excluding traces in which a correct authenticator is guessed, so that $G_{4} \approx_{\frac{N_{S}}{2^{l}}} G_{5}$ where $N_{S}$ is the number of sessions of the server $S$ interacting with the adversary. Game $G_{5}$ is transformed into $G_{6}$ by inserting event Auth', and $G_{6}$ is transformed into $G_{7}$ by inserting event AskH. Finally, one evaluates the probability of the various events in game $G_{7}$ :

$$
\begin{aligned}
& \operatorname{Pr}\left[C\left[G_{7}\right]: \text { Encrypt }\right] \leq \frac{N_{U}}{N} \\
& \operatorname{Pr}\left[C\left[G_{7}\right]: \text { Auth }^{\prime}\right] \leq \frac{N_{S}}{N} \\
& \operatorname{Pr}\left[C\left[G_{7}\right]: \text { AskH }\right] \leq q_{h} \text { Succ }_{\mathbb{G}}^{\text {cdh }}\left(t^{\prime}\right) \\
& \operatorname{Pr}\left[C\left[G_{7}\right]: \mathrm{S}\right]=\operatorname{Pr}\left[C\left[G_{7}\right]: \overline{\mathrm{S}}\right]
\end{aligned}
$$

where the password in chosen in a dictionary of size $N, N_{U}$ is the number of sessions of the client $U$ interacting with the adversary, $N_{S}$ is the number of sessions of the server $S$ interacting with the adversary, $N_{P}$ is the number of sessions between the client $U$ and server $S$ that the adversary passively eavesdrops, $t^{\prime} \leq t_{C}+\left(2 N_{U}+N_{S}+2 N_{P}+q_{e}+1\right) \tau_{\text {exp }}$, with $q_{e}$ denoting the number encryption/decryption queries asked by the adversary and $\tau_{\text {exp }}$ denoting the computation time for an exponentiation in $\mathbb{G}$.

From this proof, we can bound the advantage $\operatorname{Adv}_{\text {oeke }}^{\text {ake }}(C)$ in $G_{0}$. Let $\operatorname{Adv}_{G}^{\text {ake }}(C, D)=\operatorname{Pr}[C[G]: \mathrm{S} \vee D]-\operatorname{Pr}[C[G]: \overline{\mathrm{S}}]$ as in Lemma 2. (Here, $G$ already includes the test queries, so we need not compose with $R_{x}$ in parallel; the event NonUnique never occurs, so we omit it.) By Lemma 2,

$$
\begin{aligned}
& \operatorname{Adv}_{\text {oeke }}^{\text {ake }}(C)=\operatorname{Adv}_{G_{0}}^{\text {ake }}(C, \text { false }) \\
& \leq 2 p_{\text {collo } 0}+\operatorname{Adv}_{G_{3}}^{\text {ake }}(C, \text { false }) \\
& \text { where } p_{\text {collo }}=\frac{q_{\mathcal{E}}^{2}}{2(q-1)}+\frac{q_{S} q_{\mathcal{E}}}{q-1}+\frac{2 q_{\mathcal{E}}^{2}+q_{S}^{2}}{2(q-1)}+\frac{q_{h}^{2}}{2^{l_{1}+1}} \\
& \operatorname{Adv}_{\text {oeke }}^{\text {ake }}(C) \\
& \leq 2 p_{\text {collo }}+\operatorname{Adv}_{G_{4}}^{\text {ake }}(C, \text { Encrypt }) \\
& \leq 2 p_{\text {collo } 0}+\frac{2 N_{S}}{2^{l_{1}}}+\operatorname{Adv}_{G_{5}}^{\text {ake }}(C, \text { Encrypt }) \\
& \leq 2 p_{\text {collo }}+\frac{2 N_{S}}{2^{l_{1}}}+\operatorname{Adv}_{G_{6}}^{\text {ake }}\left(C, \text { Encrypt } \vee \text { Auth }^{\prime}\right) \\
& \leq 2 p_{\text {collo }}+\frac{2 N_{S}}{2^{l_{1}}}+\operatorname{Adv}_{G_{7}}^{\text {ake }}\left(C, \text { Encrypt } \vee \text { Auth }^{\prime} \vee \text { AskH }\right) \\
& \leq 2 p_{\text {collo }}+\frac{2 N_{S}}{2^{l_{1}}}+\operatorname{Pr}\left[C\left[G_{7}\right]: \text { Encrypt } \vee \text { Auth }^{\prime} \vee \text { AskH }\right] \\
& \leq 2 p_{\text {collo }}+\frac{2 N_{S}}{2^{l_{1}}}+\frac{N_{U}}{N}+\frac{N_{S}}{N}+q_{h} \operatorname{Succ}_{\mathbb{G}}^{\mathrm{cdh}}\left(t^{\prime}\right)
\end{aligned}
$$


Moreover,

$$
\begin{aligned}
p_{\text {coll } 0} & =\frac{q_{\mathcal{E}}^{2}+2 q_{S} q_{\mathcal{E}}+2 q_{\mathcal{E}}^{2}+q_{S}^{2}}{2(q-1)}+\frac{q_{h}^{2}}{2^{l_{1}+1}} \\
& \leq \frac{\left(2 q_{\mathcal{E}}+q_{S}\right)^{2}}{2(q-1)}+\frac{q_{h}^{2}}{2^{l_{1}+1}} \\
& \leq \frac{\left(2 q_{e}+2 N_{U}+3 N_{S}+3 N_{P}\right)^{2}}{2(q-1)}+\frac{q_{h}^{2}}{2^{l_{1}+1}}
\end{aligned}
$$

since $q_{\mathcal{E}} \leq q_{e}+N_{U}+N_{S}+N_{P}$ and $q_{S} \leq N_{S}+N_{P}$. So

$$
\begin{aligned}
\operatorname{Adv}_{\text {oeke }}^{\text {ake }}(C) \leq & \frac{N_{U}+N_{S}}{N}+q_{h} \operatorname{Succ}_{\mathbb{G}}^{\text {cdh }}\left(t^{\prime}\right)+ \\
& \frac{\left(2 q_{e}+2 N_{U}+3 N_{S}+3 N_{P}\right)^{2}}{q-1}+\frac{q_{h}^{2}+2 N_{S}}{2^{l_{1}}}
\end{aligned}
$$

Similarly, for unilateral authentication [55, Section 3.3], we use an event Auth executed when the adversary submits an authenticator accepted by the server and built by the adversary itself, so the probability for an adversary $C$ to make a server instance accept with no terminating client partner is

$$
\operatorname{Adv}_{\text {oeke }}^{\text {c-auth }}(C)=\operatorname{Pr}\left[C\left[G_{0}\right]: \text { Auth }\right]
$$

We obtain similarly by Lemma 1

$$
\begin{aligned}
& \operatorname{Pr}\left[C\left[G_{0}\right]: \text { Auth }\right] \leq p_{\text {collo } 0}+\frac{N_{S}}{2^{l_{1}}}+ \\
& \operatorname{Pr}\left[C\left[G_{7}\right]: \text { Auth } \vee \text { Encrypt } \vee \text { Auth }^{\prime} \vee \text { AskH }\right]
\end{aligned}
$$

Since $G_{7}$ never executes event Auth,

$$
\begin{aligned}
& \operatorname{Pr}\left[C\left[G_{7}\right]: \begin{array}{l}
\text { Auth } \vee \text { Encrypt } \\
\vee \text { Auth } \\
\end{array} \text { AskH }\right] \leq \frac{N_{U}}{N}+\frac{N_{S}}{N}+q_{h} \operatorname{Succ}_{\mathbb{G}}^{\text {cdh }}\left(t^{\prime}\right) \\
& \text { so } \\
& \operatorname{Adv}_{\text {oeke }}^{\text {c-auth }}(C) \leq \frac{N_{U}+N_{S}}{N}+q_{h} \operatorname{Succ}_{\mathbb{G}}^{\text {cdh }}\left(t^{\prime}\right)+ \\
& \qquad \frac{\left(2 q_{e}+2 N_{U}+3 N_{S}+3 N_{P}\right)^{2}}{2(q-1)}+\frac{q_{h}^{2}+2 N_{S}}{2^{l_{1}+1}}
\end{aligned}
$$

APPENDIX E.

\section{New Game Transformations}

The CryptoVerif proof of OEKE requires new game transformations that we have implemented. This appendix describes these games transformations. For a better understanding, we recommend reading Appendix A before reading this appendix.

\section{A. The transformation move array}

The transformation move array $X$ delays the generation of a random value $X$ until the point at which it is first used. This transformation is implemented as a particular case of a cryptographic transformation by the following equivalence:

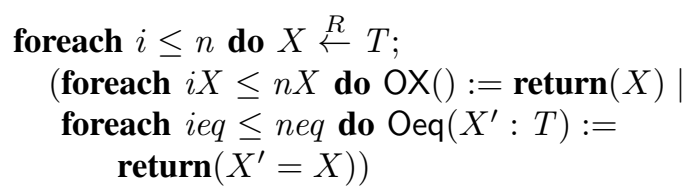

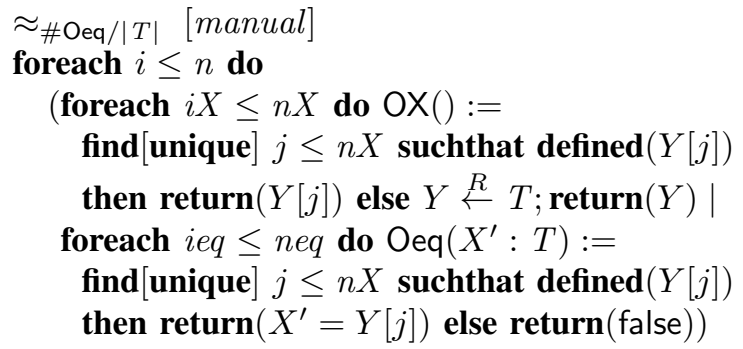

where $T$ is the type of $X$. Two oracles are defined, OX and Oeq. In the left-hand side, OX returns the random $X$ itself. In the right-hand side, OX uses a lookup to test if the random value was already generated; if yes, it returns the previously generated random value $Y[j]$; if no, it generates a fresh random value $Y$. Transforming the left-hand side into the right-hand side therefore moves the generation of the random number $X$ to the first call to OX, that is, the first usage of $X$. The oracle Oeq provides an optimized treatment of equality tests $X^{\prime}=X$ : when the random value $X$ was not already generated, we return false instead of generating a fresh $X$, so we exclude the case that $X^{\prime}$ is equal to a fresh $X$. This case has probability $1 /|T|$ for each call to Oeq, so the probability of distinguishing the two games is $\#$ Oeq $/|T|$. (Notice that, similarly to the reasoning done in Section III-B for the Random Oracle Model, there never exist several choices of $j$ that satisfy the conditions of the finds in the right-hand side of this equivalence, so these finds can be marked [unique] without modifying their behavior.)

\section{B. Extensions of simplification}

We have also extended simplification with the following transformations:

1) If one of the then branches of a find[unique] always succeeds and the conditions of this find[unique] do not contain event_abort, then we keep only that branch. Indeed, the other branches are never taken: the conditions of this find[unique] never abort, and the find itself aborts when there are several successful choices.

2) We reorganize a find[unique] that occurs in a then branch of a find[unique], when conditions of these find[unique] do not contain event_abort: we transform

$$
\begin{gathered}
\text { find }[\text { unique }]\left(\bigoplus_{j=1}^{k} \tilde{u}_{j}=\tilde{i}_{j} \leq \tilde{n}_{j} \text { suchthat } c_{j}\right. \\
\text { then } \left.P_{j}\right) \text { else } P
\end{gathered}
$$

where $P_{j_{0}}=$ find $[$ unique $]\left(\bigoplus_{j^{\prime}=1}^{k^{\prime}} F B_{j^{\prime}}\right)$ else $P_{j_{0}}^{\prime \prime}$ into 


$$
\begin{aligned}
& \text { find }\left[\text { unique] } \left(\bigoplus_{j=1 . . k, j \neq j_{0}} \begin{array}{l}
\tilde{u}_{j}=\tilde{i}_{j} \leq \tilde{n}_{j} \\
\text { suchthat } \left.c_{j} \text { then } P_{j}\right)
\end{array}\right.\right. \\
& \oplus\left(\bigoplus_{j^{\prime}=1}^{k^{\prime}} \bigoplus_{\left(\tilde{u}^{\prime}=\tilde{i}^{\prime} \leq \tilde{n}^{\prime} \text { suchthat } c^{\prime} \text { then } P^{\prime}\right) \in \mathrm{b}\left(F B_{j^{\prime}}, L_{j^{\prime}}\right)}\right. \\
& \tilde{u}_{j_{0}}=\tilde{i}_{j_{0}} \leq \tilde{n}_{j_{0}}, \tilde{u}^{\prime}=\tilde{i}^{\prime} \leq \tilde{n}^{\prime} \\
& \text { suchthat } \left.c_{j_{0}} \wedge c^{\prime} \text { then } P^{\prime}\right) \\
& \text { else find }\left[\text { unique] } \tilde{u}_{j_{0}}=\tilde{i}_{j_{0}} \leq \tilde{n}_{j_{0}} \text { suchthat } c_{j_{0}}\right. \\
& \text { then } P_{j_{0}}^{\prime \prime} \text { else } P
\end{aligned}
$$

where

$$
-F B_{j^{\prime}}=\left(\tilde{u}_{j^{\prime}}^{\prime}=\tilde{i}_{j^{\prime}}^{\prime} \leq \tilde{n}_{j^{\prime}}^{\prime} \text { suchthat } c_{j^{\prime}}^{\prime} \text { then } P_{j^{\prime}}^{\prime}\right)
$$

$-c_{j^{\prime}}^{\prime}=\operatorname{defined}\left(\tilde{M}_{j^{\prime}}\right) \wedge \ldots$

$-\tilde{i}$ are the current replication indices at the transformation point

$-L_{j^{\prime}}$ is the list of $x[\tilde{N}]$ subterm of $\tilde{M}_{j^{\prime}}$ with $x \in \tilde{u}_{j_{0}}$ and $\tilde{N} \neq \tilde{i}$, ordered by increasing size

$-\mathrm{b}\left(\tilde{u}^{\prime}=\tilde{i}^{\prime} \leq \tilde{n}^{\prime}\right.$ suchthat $c^{\prime}$ then $\left.P^{\prime},[]\right)=$ $\left\{\tilde{u}^{\prime}={\tilde{i^{\prime}}}^{\prime} \leq \tilde{n}^{\prime}\right.$ suchthat $c^{\prime}\left\{\tilde{i}_{j_{0}} / \tilde{u}_{j_{0}}\right\}$ then $\left.P^{\prime}\right\}$

$-\mathrm{b}(F B, x[\tilde{N}]:: L)=\mathrm{b}(F B, L) \cup$

$\left\{\tilde{u}^{\prime}=\tilde{i}^{\prime} \leq \tilde{n}^{\prime}\right.$ suchthat $c^{\prime}\{i / x[\tilde{N}]\} \wedge \tilde{N}=\tilde{i}$ then $P^{\prime}$

$\mid\left(\tilde{u}^{\prime}=\tilde{i}^{\prime} \leq \tilde{n}^{\prime}\right.$ suchthat $c^{\prime}$ then $\left.\left.P^{\prime}\right) \in \mathrm{b}(F B, L)\right\}$

where $i=x\left\{\tilde{i}_{j_{0}} / \tilde{u}_{j_{0}}\right\}$.

The function $b$ takes into account that, before the transformation, $\tilde{u}_{j_{0}}[\tilde{i}]$ is defined when we test defined $\left(\tilde{M}_{j^{\prime}}\right)$ in $F B_{j^{\prime}}$, while after the transformation, $\tilde{u}_{j_{0}}[\tilde{i}]$ is not defined yet when we perform this test. Furthermore, the value of $\tilde{u}_{j_{0}}[\tilde{i}]$ will be $\tilde{i}_{j_{0}}$. Therefore, 1) when we access $x[\tilde{i}]$ in $c_{j^{\prime}}^{\prime}=\operatorname{defined}\left(\tilde{M}_{j^{\prime}}\right) \wedge \ldots$, we replace this access with $i$, where $i=x\left\{\tilde{i}_{j_{0}} / \tilde{u}_{j_{0}}\right\}$; this is done in $\mathrm{b}(F B,[])$ by the substitution $\left\{\tilde{i}_{j_{0}} / \tilde{u}_{j_{0}}\right\}$; and 2) when we access $x[\tilde{N}]$ in $c_{j^{\prime}}^{\prime}=\operatorname{defined}\left(\tilde{M}_{j^{\prime}}\right) \wedge$ ... for $\tilde{N}$ not syntactically equal to $\tilde{i}$, we need to distinguish two cases: either at runtime $\tilde{N}=\tilde{i}$ and we replace this access with $i$ (second part of the union in $\mathrm{b}(F B, x[\tilde{N}]:: L))$, or at runtime $\tilde{N} \neq \tilde{i}$ and we continue using $x[\tilde{N}]$ (first part of the union in $\mathrm{b}(F B, x[\tilde{N}]:: L))$. The array accesses $x[\tilde{N}]$ in $L_{j^{\prime}}$ are ordered by increasing size because, in case of nested array accesses, we need to handle the bigger array access first (so it must occur last in the list), because after substitution of the smaller one with $i$, we would not recognize the bigger one.

This transformation cannot be performed when the outer find is not unique because it might change the probability of taking each branch. Moreover, we tried performing such a transformation when the inner find is not unique (in this case, after transformation, the outer find is not unique), but it had a negative impact in some examples. Furthermore, in the latter case, the transformation can be performed manually by inserting the desired outer find and simplifying the game: CryptoVerif will remove the useless branches of find.

After this transformation, we advise renaming the variables $\tilde{u}_{j_{0}}$ to distinct names, since they now have multiple definitions.

3) We reorganize a find [unique] that occurs in a condition of a find: we transform

$$
\begin{gathered}
\text { find }\left[\text { unique?] } \left(\bigoplus_{j=1}^{k} \tilde{u}_{j}=\tilde{i}_{j} \leq \tilde{n}_{j} \text { suchthat } c_{j}\right.\right. \\
\text { then } \left.P_{j}\right) \text { else } P
\end{gathered}
$$

where

$$
\begin{aligned}
& c_{j_{0}}=\operatorname{defined}\left(\tilde{M}^{\prime}\right) \wedge \text { find }[\text { unique }] \\
& \quad\left(\bigoplus_{j^{\prime}=1}^{k^{\prime}} \tilde{u}_{j^{\prime}}^{\prime}=\tilde{i}_{j^{\prime}}^{\prime} \leq \tilde{n}_{j^{\prime}}^{\prime} \text { suchthat } c_{j^{\prime}}^{\prime} \text { then } M_{j^{\prime}}^{\prime}\right) \\
& \text { else false }
\end{aligned}
$$

and $c_{j_{0}}$ does not contain event_abort into

$$
\begin{gathered}
\text { find }\left[\text { unique?] } \left(\bigoplus_{j=1 . . k, j \neq j_{0}} \begin{array}{r}
\tilde{u}_{j}=\tilde{i}_{j} \leq \tilde{n}_{j} \text { suchthat } \\
\left.c_{j} \text { then } P_{j}\right)
\end{array}\right.\right. \\
\oplus\left(\bigoplus_{j^{\prime}=1}^{k^{\prime}} \tilde{u}_{j_{0}}=\tilde{i}_{j_{0}} \leq \tilde{n}_{j_{0}}, \tilde{u}_{j^{\prime}}^{\prime}=\tilde{i}_{j^{\prime}}^{\prime} \leq \tilde{n}_{j^{\prime}}^{\prime}\right. \text { suchthat } \\
\left.\operatorname{defined}\left(\tilde{M}^{\prime}\right) \wedge c_{j^{\prime}}^{\prime} \wedge M_{j^{\prime}}^{\prime} \text { then } P_{j_{0}}\right)
\end{gathered}
$$

else $P$

The indication [unique?] corresponds to either [unique] or empty. The find is marked [unique] after transformation if the outer find was [unique] before transformation.

For all these transformations, the correctness proof shows that, when the initial game does not execute event NonUnique, the transformed game behaves in the same way as the initial game. We can then apply point 2 of Lemmas 1 and 2 to bound the probability of attack.

\section{The transformation merge_arrays}

The transformation merge_arrays $x_{11} \ldots x_{1 n}, \ldots$, $x_{m 1} \ldots x_{m n}$ merges the variables $x_{j 1}, \ldots, x_{j n}$ into a single variable $x_{j 1}$ for each $j \leq m$. Each variable $x_{j k}$ must have a single definition. For each $j \leq n$, the variables $x_{j 1}, \ldots, x_{j n}$ must have the same type and indices of the same type. They must not be defined for the same value of their indices (that is, $x_{j k}$ and $x_{j k^{\prime}}$ must be defined in different branches of if or find when $k \neq k^{\prime}$ ). The arrays $x_{j 1}, \ldots, x_{j n}$ are merged into a single array $x_{j 1}$ for each $j \leq m$. The transformation proceeds as follows:

- If, for each $k \leq n, x_{1 k}$ is defined above $x_{j k}$ for all $1<j<m$, we introduce a fresh variable $b_{k}$ defined by $b_{k} \leftarrow$ mark just after the definition of $x_{1 k}$. We call $b_{k}$ a branch variable; it is used to detect that $x_{j k}$ has been defined: $x_{j k}[\tilde{M}]$ is defined before the transformation if and only if $x_{j 1}[\tilde{M}]$ and $b_{k}[\tilde{M}]$ are defined after the 
transformation, and $x_{j 1}[\tilde{M}]$ after the transformation is equal to $x_{j k}[\tilde{M}]$ before the transformation.

- For each find that requires that some variables $x_{j k}$ are defined, we leave the branches that do not require the definition of $x_{j k}$ unchanged and we try to transform the other branches $F B_{l}=\left(\tilde{u}_{l}=\tilde{i}_{l} \leq\right.$ $\tilde{n}_{l}$ suchthat defined $\left(\tilde{M}_{l}\right) \wedge M_{l}$ then $\left.P_{l}\right)$ as follows.

1) We require that, for each $l$, there exists a distinct $k$ such that the defined condition of $F B_{l}$ refers to $x_{j k}$ for some $j$ but not to $x_{j k^{\prime}}$ for any other $k^{\prime}$. (Otherwise, the transformation fails.) We denote by $l(k)$ the value of $l$ that corresponds to $k$.

2) We choose a "target" branch $F B_{\mathrm{T}}=(\tilde{u}=$ $\tilde{i} \leq \tilde{n}$ suchthat defined $(\tilde{M}) \wedge M$ then $P$ ): if the defined condition of some branch $F B_{l}$ refers to $x_{j 1}$ for some $j$, we choose that branch $F B_{l}$. Otherwise, we choose any branch $F B_{l}$ and rename its variables $x_{j k}$ to $x_{j 1}$. We require that the references $x_{j 1}[\tilde{M}]$ to the variables $x_{j 1}$ in the defined condition of the target branch all have the same indices $\tilde{M}$. If the transformation succeeds, we will replace all branches $F B_{l}$ with the target branch.

3) The branch $F B_{\mathrm{T}}$ after transformation is equivalent to branches $\bigoplus_{k=1}^{n} F B_{\mathrm{T}}\left\{x_{j k} / x_{j 1}, j=\right.$ $1 \ldots m\}$ before transformation. We show that these branches are equivalent to the branches $F B_{l}$.

For each $k \leq n$,

- if $l(k)$ exists, then we show that $F B_{\mathrm{T}}\left\{x_{j k} / x_{j 1}\right.$, $j=1 \ldots m\}$ is equivalent to $F B_{l(k)}$. Let $l=l(k)$. We first rename the variables $\tilde{u}_{l}$ of $F B_{l}$ to the variables $\tilde{u}$ of the target branch. For simplicity, we still denote by $F B_{l}=$ $\left(\tilde{u}_{l}=\tilde{i}_{l} \leq \tilde{n}_{l}\right.$ suchthat $\operatorname{defined}\left(\tilde{M}_{l}\right) \wedge M_{l}$ then $P_{l}$ ) the obtained branch. Then we show that, if the variables of $\tilde{M}_{l}$ are defined, then the variables of $\tilde{M}\left\{x_{j k} / x_{j 1}, j=1 \ldots m\right\}$ are defined, and conversely; $M_{l}=M\left\{x_{j k} / x_{j 1}\right.$, $j=1 \ldots m\}$ (knowing the equalities that hold at that program point), and $P_{l}$ and $P\left\{x_{j k} / x_{j 1}\right.$, $j=1 \ldots m\}$ execute the same code up to renaming of variables defined in $P_{l}$ or $P\left\{x_{j k} / x_{j 1}\right.$, $j=1 \ldots m\}$ and that do not have array accesses.

- if $l(k)$ does not exist, then we show that $F B_{\mathrm{T}}\left\{x_{j k} / x_{j 1}, j=1 \ldots m\right\}$ can in fact not be executed, because its condition cannot hold: the variables of $\tilde{M}\left\{x_{j k} / x_{j 1}, j=1 \ldots m\right\}$ cannot be simultaneously defined or $M\left\{x_{j k} / x_{j 1}, j=\right.$ $1 . . . m\}$ cannot hold.

If the transformation above fails and we have introduced branch variables, we replace each condition $\operatorname{defined}\left(x_{j k}[\tilde{M}]\right)$ with $\operatorname{defined}\left(x_{j 1}[\tilde{M}], b_{k}[\tilde{M}]\right)$.

If the transformation above fails and we have not introduced branch variables, the whole merge_arrays transformation fails.

- The definition of $x_{j k}$ is renamed to $x_{j 1}$ and each reference to $x_{j k}[\tilde{M}]$ is renamed to $x_{j 1}[\tilde{M}]$.

\section{The transformation merge_branches}

The transformation merge_branches performs the following transformations:

1) If some then branches of a find[unique] execute the same code as the else branch (up to renaming of variables defined in these branches and that do not have array accesses), the variables bound in the condition of these then branches have no array accesses, and the conditions of these then branches do not contain event_abort, then we remove these then branches. Indeed, these then branches have the same effect as the else branch. The hypotheses are needed for the following reasons:

- The renamed variables must not have array accesses because renaming variables that have array accesses requires transforming these array accesses. The transformation merge_arrays presented in Section E-C can rename variables with array accesses.

- The variables bound in conditions of the removed branches must not have array accesses, because removing the definitions of these variables would modify the behavior of the array accesses.

- The conditions must not contain event_abort, because if they do, the find may abort while code after transformation would not abort.

2) If all branches of if or find execute the same code (up to renaming of variables defined in these branches and that do not have array accesses), and in case of find, the variables bound in the conditions of the then branches have no array accesses and the conditions of the then branches do not contain event_abort, then we replace that if or find with its else branch.

In this transformation, we ignore the array accesses that occur in the conditions of the find under consideration, since these conditions will disappear after the transformation.

Furthermore, merge_branches applies these transformations globally to all finds of the game for which the simplification is possible. As a consequence, one can ignore array accesses to all variables in conditions of find that will be removed, so more transformations are enabled. 